\author{
Universidade de São Paulo \\ Instituto de Física
}

\title{
Modelos de Mapas Simpléticos para o Movimento de Deriva Elétrica com Efeitos de Raio de Larmor Finito
}

\author{
Júlio César David da Fonseca
}

Orientador: Prof. Dr. Iberê Luiz Cadas

Tese de doutorado apresentada ao Instituto de Física para a obtenção do título de Doutor em Ciências

Banca Examinadora:

Prof. Dr. Iberê Luiz Cadas (USP)

Prof. Dr. Edson Denis Leonel (UNESP - Rio Claro)

Prof. Dr. Ricardo Egydio de Carvalho (UNESP - Rio Claro)

Prof. Dr. Ricardo Luis Viana (UFPR)

Prof. Dr. Roberto Venegeroles Nascimento (UFABC) 


\section{FICHA CATALOGRÁFICA \\ Preparada pelo Serviço de Biblioteca e Informação do Instituto de Física da Universidade de São Paulo}

\section{Fonseca, Júlio César David da}

Modelos de mapas simpléticos para o movimento de deriva elétrica com efeitos de raio de Larmor finito. São Paulo, 2016.

Tese (Doutorado) - Universidade de São Paulo. Instituto de Física Depto. de Física Aplicada.

Orientador: Prof. Dr. Iberê Luiz Caldas

Área de Concentração: Física

Unitermos: 1. Física de plasmas; 2. Fenômeno de transporte;

3. Caos (Sistemas dinâmicos). 


\title{
University of São Paulo Institute of Physics
}

\section{Area-Preserving Maps Models of the Electric Drift Motion with Finite Larmor Radius Effects}

\author{
Júlio César David da Fonseca
}

Submitted to the Institute of Physics in partial fulfillment of the requirements for the degree of Doctor in Science.

Evaluation comitte:

Prof. Dr. Iberê Luiz Cadas (USP)

Prof. Dr. Edson Denis Leonel (UNESP - Rio Claro)

Prof. Dr. Ricardo Egydio de Carvalho (UNESP - Rio Claro)

Prof. Dr. Ricardo Luis Viana (UFPR)

Prof. Dr. Roberto Venegeroles Nascimento (UFABC) 



\section{Acknowledgements}

I would like to express my thanks to my thesis advisor Prof. Iberê L. Caldas for his guidance.

I wish also to thank Dr. Diego del Castillo Negrete from the Oak Ridge National Laboratory (USA), Prof. Igor M. Sokolov from Humboldt University in Berlin (Germany), and Prof. Roberto Venegeroles from Federal University of ABC (Brazil) for their valuable suggestions.

This work was supported by the brazilian research agencies CNPq and FAPESP. 


\section{Resumo}

Mapas simpléticos têm sido amplamente utilizados para modelar o transporte caótico em plasmas e fluidos. Neste trabalho, propomos três tipos de mapas simpléticos que descrevem o movimento de deriva elétrica em plasmas magnetizados. Efeitos de raio de Larmor finito são incluídos em cada um dos mapas. No limite do raio de Larmor tendendo a zero, o mapa com frequência monotônica se reduz ao mapa de Chirikov-Taylor, e, nos casos com frequência não-monotônica, os mapas se reduzem ao mapa padrão não-twist. Mostramos como o raio de Larmor finito pode levar à supressão de caos, modificar a topologia do espaço de fases e a robustez de barreiras de transporte. Um método baseado na contagem dos tempos de recorrência é proposto para analisar a influência do raio de Larmor sobre os parâmetros críticos que definem a quebra de barreiras de transporte. Também estudamos um modelo para um sistema de partículas onde a deriva elétrica é descrita pelo mapa de frequência monotônica, e o raio de Larmor é uma variável aleatória que assume valores específicos para cada partícula do sistema. A função densidade de probabilidade para o raio de Larmor é obtida a partir da distribuição de Maxwell-Boltzmann, que caracteriza plasmas na condição de equilíbrio térmico. Um importante parâmetro neste modelo é a variável aleatória gama, definida pelo valor da função de Bessel de ordem zero avaliada no raio de Larmor da partícula. Resultados analíticos e numéricos descrevendo as principais propriedades estatísticas do parâmetro gama são apresentados. Tais resultados são então aplicados no estudo de duas medidas de transporte: a taxa de escape e a taxa de aprisionamento por ilhas de período um. 


\section{Abstract}

Area-preserving maps have been extensively used to model chaotic transport in plasmas and fluids. In this work we propose three types of maps describing electric drift motion in magnetized plasmas. Finite Larmor radius effects are included in all maps. In the limit of zero Larmor radius, the monotonic frequency map reduces to the Chirikov-Taylor map, and, in cases with non-monotonic frequencies, the maps reduce to the standard nontwist map. We show how the finite Larmor radius can lead to chaos suppression, modify the phase space topology and the robustness of transport barriers. A method based on counting the number of recurrence times is used to quantify the dependence on the Larmor radius of the threshold for the breakup of transport barriers. We also study a model for a system of particles where the electric drift is described by the monotonic frequency map, and the Larmor radius is a random variable that takes a specific value for each particle of the system. The Larmor radius' probability density function is obtained from the Maxwell-Boltzmann distribution, which characterizes plasmas in thermal equilibrium. An important parameter in this model is the random variable gamma, defined by the zero-order Bessel function evaluated at the Larmor radius'particle. We show analytical and numerical computations related to the statistics of gamma. The set of analytical results obtained here is then applied to the study of two numerical transport measures: the escape rate and the rate of trapping by period-one islands. 


\section{Contents}

1 Introduction 1

2 Gyro-Averaged E $\times$ B Maps and FLR Effects $\quad 7$

2.1 Gyro-averaged $\vec{E} \times \vec{B}$ Drift Model . . . . . . . . . . . . . . . . 7

2.2 Gyro-averaged standard map $(\mathrm{GSM}) \ldots \ldots \ldots$

2.3 Gyro-averaged standard nontwist map $(\mathrm{GSNM}) \ldots \ldots$. . . . . . . 12

2.3.1 Fixed Points . . . . . . . . . . . . . . . . . . 14

2.3.2 Separatrix reconnection . . . . . . . . . . . . . . 15

2.3.3 Nontwist transport barrier . . . . . . . . . . . . . . . . . . . . 19

2.3.4 Breakup Diagrams . . . . . . . . . . . . . . . 20

2.4 Gyro-averaged quartic nontwist map $(\mathrm{GQNM}) \ldots \ldots \ldots$. . . . . . 29

2.4.1 Fixed Points and nontwist transport barriers . . . . . . . . . . . . . 31

2.4.2 Robustness of the central shearless curve . . . . . . . . . . . . 33

3 Statistical Properties of the GSM Model 39

3.1 GSM Model . . . . . . . . . . . . . . . . . . . . . . . . . . . 39

3.1.1 Maxwell-Boltzmann Distribution . . . . . . . . . . . . . . . 40

3.1.2 Larmor Radius' PDF . . . . . . . . . . . . . . . . . . . . . . . 41

3.2 Gamma's Probability Density Function . . . . . . . . . . . . . . . . . 43

3.3 Gamma's Average and Dispersion . . . . . . . . . . . . . . 47

3.4 Gamma's Cumulative Distribution Function . . . . . . . . . . . . . . . 49

3.5 Probability of Global Chaos $\left(P_{c}\right) \ldots \ldots \ldots \ldots$

3.6 Escape Rate . . . . . . . . . . . . . . . . . . . 56 
3.7 Rate of Trapping by Period-One Islands . . . . . . . . . . . . . . . . . . 60

$\begin{array}{lll}4 \text { Conclusion } & 69\end{array}$

$\begin{array}{ll}\text { Appendices } & 75\end{array}$

$\begin{array}{ll}\text { A Gyro-averaged Drift Wave Map } & 77\end{array}$

$\begin{array}{lr}\text { B Indicator Points } & 81\end{array}$

B.1 GSNM Indicator Points . . . . . . . . . . . . . . . . . . . 81

B.1.1 Involutions and Symmetry . . . . . . . . . . . . . . . . . 81

B.1.2 Fixed Points of $S I_{0}$ and $S I_{1} \ldots \ldots \ldots \ldots$

B.2 GQNM Indicator Points . . . . . . . . . . . . . . . . . . . 85

$\begin{array}{ll}\text { C Statistics of Gamma: Further Results } & 87\end{array}$

C.1 Alternative form of the Gamma's PDF . . . . . . . . . . . . . . . 87

C.2 Moments. . . . . . . . . . . . . . . . . . . . . 94

C.3 Cumulative Distribution Function . . . . . . . . . . . . . . . . 95 


\section{Chapter 1}

\section{Introduction}

Important research efforts in controlled nuclear fusion are focused on the magnetic confinement of hot plasmas. In order to improve the confinement conditions, a better understanding of the particle transport is needed, specially in the case of electric drift motion or, simply, $\vec{E} \times \vec{B}$ transport. A typical approach to this problem is based on the $\vec{E} \times \vec{B}$ approximation of charged particle's guiding center's motion [1-4]. However, in case of fast particles (e.g. alpha particles in burning plasmas) or inhomogeneous fields on the scale of the Larmor radius, it is necessary to consider finite Larmor radius (FLR) effects $[5,6]$.

Previous studies on the role of the Larmor radius include Refs. $[5,7,8]$, where nondiffusive particle transport in numerical simulations of eletrostatic turbulence was analyzed and FLR effects were shown to inhibit transport. Non-diffusive chaotic transport was studied in [9] using an $\vec{E} \times \vec{B}$ Hamiltonian model that incorporates FLR effects. In $[6,10]$, the authors studied another Hamiltonian model of the $\vec{E} \times \vec{B}$ transport with FLR effects, making use of dynamical systems methods to investigate Larmor radius's influence on the formation of complex phase space topologies and chaos supression.

In this work we analyse FLR effects in simple area preserving maps models of $\vec{E} \times \vec{B}$ motion. The maps presented here are constructed following the Hamiltonian framework in [11] for electrostatic drift motion. The Hamiltonian is determined by a time dependent electrostatic potential, which depends on the radial and the poloidal coordinates. FLR corrections are included into the model by gyro-averaging [12] the electrostatic potential. 
The electrostatic potential consists of two parts: the first one depends only on the radial coordinate and is called the equilibrium potential; the second part, which depends on time and the poloidal coordinate, represents a superposition of drift waves. We model the drift waves following the approach in [13] that allows the contruction of area preserving maps as simple discrete models of $\vec{E} \times \vec{B}$ transport. Depending on the equilibrium potential, the maps' frequencies exhibit monotonic or non-monotonic profiles. Using these simple maps models of gyro-averaged chaotic $\vec{E} \times \vec{B}$ transport, we analyze the role of Larmor radius in the following problems: chaos supression, stability of fixed points, nontwist phase space topologies, and transport barriers.

The term "nontwist" is used to designate Hamiltonian systems that violate the twist or nondenegeracy property. That is the case for Hamiltonian systems whose frequency is a non-monotonic function of the action variable. Nontwist Hamiltonian systems are characterized by particular phase space topologies [14] and can be found in many different physical models. Some examples include: $\mathbf{E} \times \mathbf{B}$ transport in magnetized plasmas $[3,4$, 15]; magnetic fields with reverse shear in toroidal plasma devices [16-18]; modelling of transport by traveling waves in shear flows in fluids [19-21].

The presence of nontwist transport barriers is among the most important properties exhibited by nontwist Hamiltonian systems. By nontwist transport barriers we mean a robust region of spanning Kolmogorov-Arnold-Moser (KAM) curves that are resistant to "breakup", i.e. they can survive even when the phase space is almost completely dominated by chaotic dynamics. In Hamiltonian dynamical systems theory, spanning KAM curves, also called KAM barriers [19] or rotational invariant circles [22], are known to divide the phase space in such a way that chaotic orbits become confined among them. For this reason, spanning KAM curves are interesting in the study of transport problems, specially those related to $\mathbf{E} \times \mathbf{B}$ Hamiltonian models. In the context of one and a half degrees of freedom nontwist Hamiltonian systems, the robusteness of spanning KAM curves has also been called strong KAM stability [23] and has been the subject of several studies (see, for example, [24-27] and references therein).

In this work the maps correspond to Hamiltonians whose frequencies can be monotonic or non-monotonic functions of the action variable. The latter case are characterized by the 
presence of nontwist transport barriers that can be destroyed or restored by changing the value of the Larmor radius. This feature is directly related to the FLR effect of chaotic transport suppression, whose study was initiated by $[6,10]$. Here we show that chaos suppression occurs when the Larmor radii are close to specific values, for which invariant circles become very resilient to breakup. In the case of the nontwist maps, the invariant circles that constitute the nontwist transport barrier are the most easily restored and hardest to break.

Among the main goals of the present work is the study of the critical parameters related to the destruction of nontwist barriers. These thresholds can be aproximately determined by what we call here the breakup diagrams. In order to efficiently compute breakup diagrams, this work describes a procedure based on a technique [28] which explores the recurrence properties of a dynamical system's orbit that result from the Slater's Theorem [29]. As shown in [28], and also in the later work [30], the recurrence properties of an orbit can be used to differentiate chaotic and non-chaotic motion. A recent example of the application of this technique to the standard nontwist map [19] was presented in [31].

We also study statistical properties of a model based on the gyro-averaged standard map (GSM), the simplest area-preserving map proposed in this this work. The GSM corresponds to the map with monotonic frequency map and is a modified version of the Chirikov-Taylor or standard map [32,33].

Besides a perturbation parameter, the GSM has a dependence on a function gamma, given by the zero-order Bessel function of the Larmor radius. The GSM's effective perturbation consists in the product between the perturbation parameter and gamma. In the limit of zero Larmor radius, the GSM corresponds exactly to the standard map.

In the GSM model initially analyzed, we have assumed the Larmor radius as a parameter and our goal was to study non-linear dynamics properties emerging from changing the value of this parameter. After concluding this study, we adapted the GSM model in order to consider an ensemble of charged particles, each one having its own Lamor radius. Following [6,9], the Larmor radius' probability density function (pdf) results from a Maxwell-Boltzmann distribution, which describes plasmas in thermal equilibrium.

Particles have different Larmor radii and their orbits are computed by iterations of 
different GSMs, each one with its own effective perturbation. Thus, particles "see" different phase space topologies: a particle can be trapped inside an stability island as other one, after crossing the orbit of the first, is able to reach far regions of the phase space following chaotic orbit.

Since the effective perturbation depends on the value of gamma, determining its basic statistical features is important to any transport study in the GSM model. Using the Larmor radius' pdf, we start by obtaining a set of analytical expressions including the pdf, the average and the dispersion of gamma. Histograms of gamma are discussed using the formulas obtained.

An analytical formula for the cumulative distribution function of gamma is also presented and verified by numerical simulations. We describe its main properties and relate them to the gamma's pdf. The cumulative distribution function of gamma is then applied to obtain two additional analytical results: the probability of global chaos and the probability of trapping by period-one islands.

The probability of global chaos is the probability of a particle moving in a phase space characterized by global chaos, i.e. a phase space where KAM barriers do not exist. The probability of trapping by period-one islands is the probability of a particle, initially located near a fixed point, being trapped by the corresponding period-one island. If the fixed point is elliptic, there is an island trapping particles near it. In case of an hyperbolic fixed point, particles, in general, move away from it following chaotic orbits.

The probabilities of global chaos and trapping are functions depending on the thermal Larmor radius and the perturbation parameter, the only two parameters in GSM model and that incorporate all relevant physics. We show plots of both probabilities, analyzing how they change regarding variations of the two parameters.

To illustrate the application of the analytical results obtained, we define and analyze two numerical transport measures: the escape rate and the rate of trapping by periodone-islands. Numerical simulations of both measures are shown and compared to the probabilites of global chaos and trapping. The role of the thermal Larmor radius and the perturbation parameter is again analyzed in detail.

The remaning part of this thesis is structured as follows. 
In chapter 2, we introduce a discrete model of the gyro-averaged $\vec{E} \times \vec{B}$ drift motion, which we call here as gyro-averaged drift wave map. The model is used to construct all maps discussed in this work. Non-linar dynamics properties resulting from FLR effects are studied for each of those maps.

In chapter 3, we focus on the statistical analysis of the GSM model.

In chapter 4 , we present a summary and concluding remarks.

Appendix A describes the details in the obtention of the gyro-averaged drift wave map.

Appendix B shows necessary conditions and computations to obtain indicator point formulas, which are important to detect the presence of nontwist transport barriers.

In the last chapter, Appendix $\mathrm{C}$, we present an alternative approach to obtain the main statistical properties of gamma and also an additional discussion about the gamma's pdf. 


\section{Chapter 2}

\section{Gyro-Averaged E $\times$ B Maps and FLR Effects}

This chapter is organized as follows. Section 2.1 describes the discrete model of gyroaveraged $\vec{E} \times \vec{B}$ drift motion, from which we derive different kinds of maps. The first example, for which the frequency has a monotonic profile, is presented in section 2.2, where FLR effects on the transition to global chaos are analyzed. Section 2.3 describes an example with a non-monotonic profile and dicusses FLR effects on the stability of fixed points, phase space topologies and breakup diagrams. Another nontwist map is analysed in section 2.4, where we turn our attention to FLR effects on zonal flow bifurcations. This chapter is partly based on Ref. [34], our last published paper.

\subsection{Gyro-averaged $\vec{E} \times \vec{B}$ Drift Model}

The $\vec{E} \times \vec{B}$ drift velocity of the guiding center is given by [35]:

$$
\vec{v}_{G C}=\frac{\vec{E} \times \vec{B}}{B^{2}} .
$$

Using $x$ as the radial coordinate and $y$ as the poloidal coordinate, the equations of the $\vec{E} \times \vec{B}$ drift motion, given by $\vec{v}_{G C}=(\dot{x}(t), \dot{y}(t))$, can be written as the Hamiltonian system:

$$
\frac{d y}{d t}=\frac{\partial H(x, y, t)}{\partial x}, \quad \frac{d x}{d t}=-\frac{\partial H(x, y, t)}{\partial y}
$$


where:

$$
H(x, y, t)=\frac{\phi(x, y, t)}{B_{0}}
$$

$\phi$ is the electrostatic potential, and we assume a constant toroidal magnetic field $\vec{B}=B_{0} \hat{e}_{z}$. As discussed in [10], finite Larmor radius (FLR) effects can be incorporated substituting the electrostatic potential by its average over a circle around the guiding center:

$$
\langle\phi(x, y, t)\rangle_{\varphi}=\frac{1}{2 \pi} \int_{0}^{2 \pi} \phi(x+\rho \cos \varphi, y+\rho \sin \varphi, t) d \varphi,
$$

where $\rho$ is the Larmor radius, which defines the radius of a charged particle's gyration around its guiding center, and the integration variable $\varphi$ is the angle of gyration. Formula (2.4) corresponds to the well-known gyro-averaging operation [12].

The gyro-averaged Hamiltonian can then be defined as:

$$
\langle H(x, y, t)\rangle_{\varphi}=\frac{\langle\phi(x, y, t)\rangle_{\varphi}}{B_{0}}
$$

and the gyro-averaged equations of motion (2.2) can be written as:

$$
\frac{d y}{d t}=\frac{\partial\langle H\rangle_{\varphi}}{\partial x}, \quad \frac{d x}{d t}=-\frac{\partial\langle H\rangle_{\varphi}}{\partial y}
$$

Following [13], we assume an electrostatic potential of the form:

$$
\phi(x, y, t)=\phi_{0}(x)+A \sum_{m=-\infty}^{+\infty} \cos \left(k y-m \omega_{0} t\right)
$$

where $\phi_{0}(x)$ is the equilibrium potential, $A$, the amplitude of the drift waves, $k$ is the wave number, and $\omega_{0}$ is the fundamental frequency.

Applying the gyro-average operation (2.4) to (2.7), and substituting the result in (2.5), we obtain the Hamiltonian:

$$
\langle H(x, y, t)\rangle_{\varphi}=\left\langle H_{0}(x)\right\rangle_{\varphi}+\frac{A}{B_{0}} J_{0}(k \rho) \sum_{m=-\infty}^{+\infty} \cos \left(k y-m \omega_{0} t\right)
$$

where $J_{0}$ is the zero-order Bessel function, and the integrable Hamiltonian $\left\langle H_{0}(x)\right\rangle_{\varphi}$ is defined as:

$$
\left\langle H_{0}(x)\right\rangle_{\varphi}=\frac{\left\langle\phi_{0}(x)\right\rangle_{\varphi}}{B_{0}}
$$


Using the Fourier series representation of the Dirac delta function, equation (2.8) can be rewritten as:

$$
\langle H(x, y, t)\rangle_{\varphi}=\left\langle H_{0}(x)\right\rangle_{\varphi}+\frac{2 \pi A}{B_{0}} J_{0}(k \rho) \cos (k y) \sum_{m=-\infty}^{+\infty} \delta\left(\omega_{0} t-2 \pi m\right) .
$$

Let $x_{n}=x\left(t_{n}^{-}\right)$and $y_{n}=y\left(t_{n}^{-}\right)$, with $t_{n}^{-}=\frac{2 \pi n}{\omega_{0}}-\varepsilon, n \in \mathbb{N}$, and $\varepsilon \rightarrow 0^{+}$. Integrating equations $(2.6)$ in the interval $\left(t_{n}^{-}, t_{n+1}^{-}\right)$leads to the gyro-averaged drift wave map ${ }^{1}$ :

$$
\begin{aligned}
& x_{n+1}=x_{n}+\frac{2 \pi k A}{\omega_{0} B_{0}} J_{0}(\hat{\rho}) \sin \left(k y_{n}\right), \\
& y_{n+1}=y_{n}+\frac{2 \pi}{\omega_{0}} \Omega\left(x_{n+1}\right),
\end{aligned}
$$

where $\hat{\rho}=k \rho$, which will call here the normalized Larmor radius, and $\Omega(x)$ corresponds to the frequency associated to the integrable Hamiltonian (2.9):

$$
\Omega(x)=\frac{d\left\langle H_{0}(x)\right\rangle_{\varphi}}{d x} .
$$

For $\rho=0, \Omega(x)=-E_{r}(x) / B_{0}$, where $E_{r}(x)$ is the radial component of the electric field. Depending on the function assumed for the equilibrium potential $\phi_{0}(x)$, which determines $E_{r}(x)$ and $\Omega(x)$, we can obtain different area preserving maps from equations (2.11) and (2.12). In the next sections, we discuss three different cases.

\subsection{Gyro-averaged standard map (GSM)}

As a first and simple example, we show how to construct the gyro-averaged standard map (GSM), assuming a monotonic linear frequency profile. The GSM is a modified version of the standard map, also known as the Chirikov-Taylor map [32,33]. To define the frequency in (2.12), we use the following form to the equilibrium potential:

$$
\phi_{0}(x)=\alpha \frac{(k x)^{2}}{2}
$$

where $k$ is the wave number in equation (2.7), and $\alpha$ is a free parameter. Applying the gyro-average operation to the equilibrium potential (2.14), we get:

$$
\left\langle H_{0}(x)\right\rangle_{\varphi}=\frac{\alpha}{B_{0}}\left[\frac{(k x)^{2}}{2}+\frac{\hat{\rho}^{2}}{4}\right] .
$$

\footnotetext{
${ }^{1}$ Details about the steps followed in this section to obtain the gyro-averaged drift wave map are presented in appendix A.
} 
Substituting (2.15) in (2.13), we get the frequency:

$$
\Omega(x)=\frac{\alpha k^{2}}{B_{0}} x
$$

which does not depend on the Larmor radius, and has a monotonic profile. Introducing the non-dimensional variables:

$$
I=k \gamma x, \quad \theta=k y
$$

and the constant:

$$
\gamma=\frac{2 \pi \alpha k^{2}}{\omega_{0} B_{0}}
$$

we get, from (2.11) and (2.12), the gyro-averaged standard map (GSM):

$$
\begin{aligned}
& I_{n+1}=I_{n}+K_{e f}(\hat{\rho}) \sin \theta_{n}, \\
& \theta_{n+1}=\theta_{n}+I_{n+1}, \quad \bmod 2 \pi
\end{aligned}
$$

where:

$$
K_{e f}=K J_{0}(\hat{\rho})
$$

is the effective perturbation parameter and $K=\gamma^{2} \frac{A}{\alpha}$ is the perturbation parameter. For $\hat{\rho}=0, K_{e f}=K$, and the GSM reduces to the standard map.

The phase space of the GSM, as in the case of any area-preserving map, is characterized by the presence of periodic, quasiperiodic, and chaotic orbits. Quasiperiodic orbits covers densely invariant curves. The invariant curves around the elliptic fixed points form island chains. The invariant curves that wind around the entire domain of the angle variable are known as rotational invariant circles [22] or, simply, as spanning KAM curves. The presence of spanning spanning KAM curves is of special interest in our model because they inhibit transport in the direction of the radial coordinate $x$, keeping chaotic orbits confined to specific regions of phase space.

According to Greene's residue method [37], the transition to global chaos in the standard map occurs when the absolute value of the perturbation parameter is equal to, or greater than, the critical value $K_{c} \simeq 0.9716$. That is, for $K \geq K_{c}$, all spanning spanning KAM curves are broken, and chaotics orbits can spread over all phase space (except in regions occupied by isolated islands), which corresponds to the situation of global chaos. 


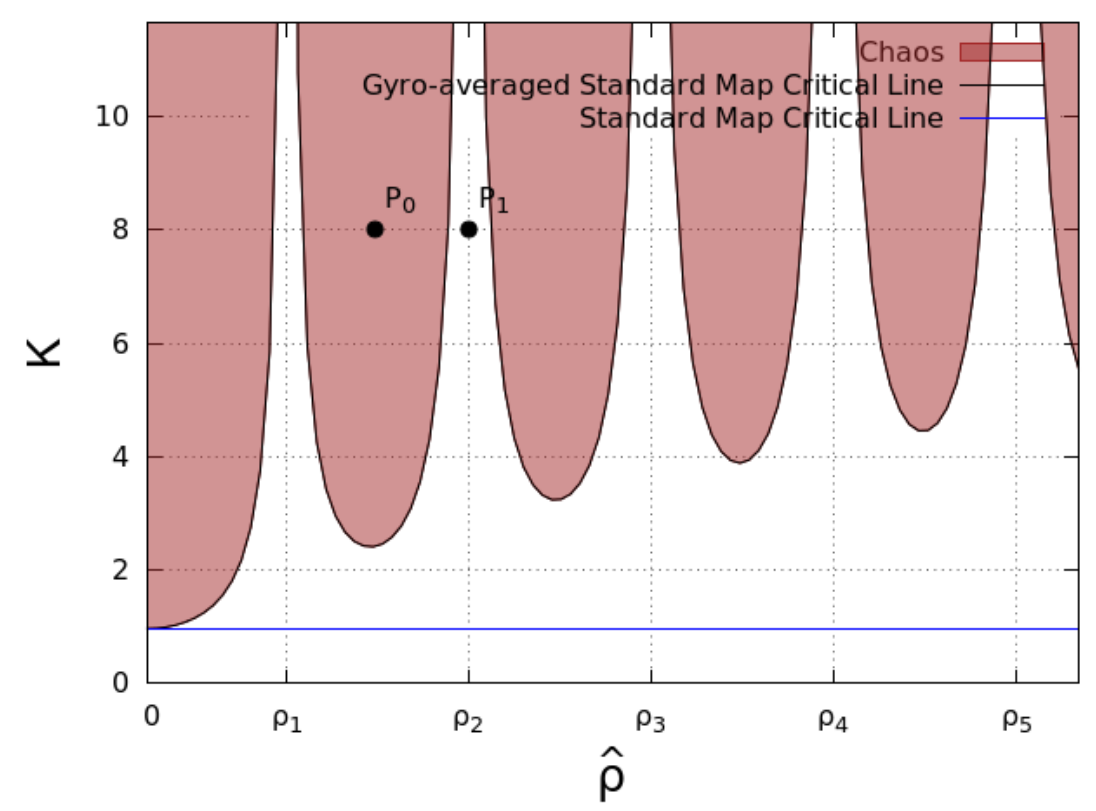

Figure 2.1: Critical lines for the standard map (blue horizontal line) and for the gyro-averaged standard map (black curves). Inside the shaded regions, there is global chaos.

Larmor radius effects on the transition to global chaos in the GSM can be analyzed by defining the critical line dividing the $K-\hat{\rho}$ parameter space in two regions: one for which the phase space contains at least one KAM curve and another for which transition to global chaos has occured. In the standard map, there is no dependence on $\hat{\rho}$, which means that the critical line is just a horizontal line defined at $K=K_{c}$, as indicated in figure 2.1. However, for the GSM, the critical line is determined by the condition $\left|K_{e f}\right|=K_{c}:$

$$
K=\frac{K_{c}}{\left|J_{0}(\hat{\rho})\right|}
$$

As shown in Fig. 2.1, even for high values of the perturbation parameter $\left(K \gg K_{c}\right)$ there are an infinite number of bands of Larmor radius values for which the spanning KAM curves can be restored and chaos is supressed.

The gyro-averaging operation "breaks" the critical line at the zeros of the zero-order Bessel function. Near the zeros, the critical perturbation goes to infinity and the transition to global chaos can not occur. The first five positive zeros, indicated in figure 2.1, are approximately $\rho_{1}=2.40, \rho_{2}=5.52, \rho_{3}=8.65, \rho_{4}=11.79$, and $\rho_{5}=14.93$. 


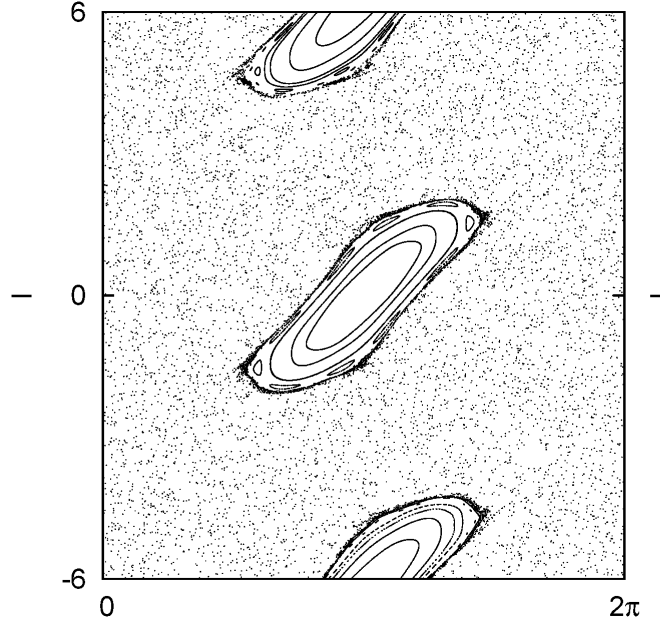

(a)

$\theta$

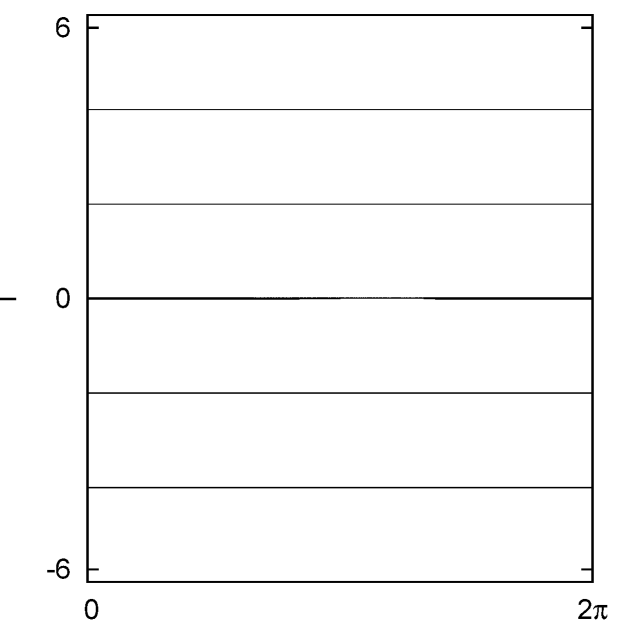

(b)

$\theta$

Figure 2.2: (a) GSM Poincaré section for $\hat{\rho}=3.9$ and $K=8.0$ (point $P_{0}$ in figure 2.1): no spanning KAM curves are observed. (b) GSM Poincaré section for $\hat{\rho}=5.52$ and $K=8.0$ (point $P_{1}$ in figure 2.1): spanning KAM curves are restored.

The effect of chaos suppression is illustrated by Figs. 2.2(a)-(b). Figure 2.2(a) shows a GSM Poincaré section for the parameter values $\hat{\rho}=3.9$ and $K=8.0$, which correspond to the point $P_{0}$ in Fig. 2.1. No spanning KAM curves are observed in the Poincaré section as $P_{0}$ belongs to the region of global chaos. Keeping the same value of $K$ and changing the $\hat{\rho}$ value to $\rho_{2}$ (point $P_{1}$ in Fig. 2.1) elliminates all the chaotic orbits (see figure 2.2(b)).

\subsection{Gyro-averaged standard nontwist map (GSNM)}

As a second example of the gyro-averaged drift wave map, we introduce the gyroaveraged standard nontwist map (GSNM), which corresponds to a non-monotonic radial eletric field. The equilibrium potential is defined by:

$$
\phi_{0}(x)=\alpha\left[\left(\frac{x}{L}\right)-\frac{1}{3}\left(\frac{x}{L}\right)^{3}\right]
$$

where $\alpha$ and $L$ are dimensional constants. The gyro-average of (2.23) results in:

$$
\left\langle H_{0}(x)\right\rangle_{\varphi}=\frac{\alpha}{B_{0}}\left[\left(\frac{x}{L}\right)\left(1-\frac{\rho^{2}}{2 L^{2}}\right)-\frac{1}{3}\left(\frac{x}{L}\right)^{3}\right]
$$


and from (2.13):

$$
\Omega(x)=\frac{\alpha}{B_{0} L}\left\{\left[1-\left(\frac{\rho}{\sqrt{2} L}\right)^{2}\right]-\left(\frac{x}{L}\right)^{2}\right\}
$$

The frequency $\Omega(x)$ has a non-monotonic parabolic profile with a maximum critical point at $x=0$. For $\rho=0$, the zeros of $\Omega(x)$ (and also of the $E_{r}$ profile) are located at $-L$ and $+L$, where $L$ is the characteristic length of the frequency profile. Substituting (2.25) into (2.12), we obtain:

$$
\begin{aligned}
& x_{n+1}=x_{n}+\frac{2 \pi}{\omega_{0} B_{0}} A k J_{0}(k \rho) \sin \left(k y_{n}\right) \\
& y_{n+1}=y_{n}+\frac{2 \pi}{\omega_{0} B_{0}} \frac{\alpha}{L}\left\{\left[1-\left(\frac{\rho}{\sqrt{2} L}\right)^{2}\right]-\left(\frac{x_{n+1}}{L}\right)^{2}\right\} .
\end{aligned}
$$

Introducing the dimensionless variables:

$$
I=-\frac{x}{L}, \quad \theta=\frac{k y}{2 \pi}
$$

we get the gyro-averaged standard nontwist map (GSNM):

$$
\begin{aligned}
& I_{n+1}=I_{n}-b J_{0}(\hat{\rho}) \sin \left(2 \pi \theta_{n}\right) \\
& \theta_{n+1}=\theta_{n}+a\left[\left(1-\frac{\bar{\rho}^{2}}{2}\right)-I_{n+1}^{2}\right], \bmod 1
\end{aligned}
$$

where:

$$
\begin{aligned}
& a=\frac{\alpha k}{\omega_{0} B_{0} L}, \quad b=\frac{2 \pi a A}{\alpha}, \\
& \bar{\rho}=\frac{\rho}{L}, \quad \hat{\rho}=k \rho,
\end{aligned}
$$

are four dimensionless parameters. We refer to $b$ as the perturbation parameter, which is proportional to the amplitude $A$ of the drift waves. Like in the GSM case, we can also define an effective perturbation parameter:

$$
b_{e f}=b J_{0}(\hat{\rho}) \text {. }
$$

The parameters $\hat{\rho}$ and $\bar{\rho}$, as indicated by equations (2.32), correspond to the Larmor radius $\rho$ normalized using two different length scales: $\hat{\rho}$ is proportional to the ratio between $\rho$ and the wave length $\lambda=\frac{2 \pi}{k}$, and $\bar{\rho}$ is the ratio between $\rho$ and the characteristic length of the frequency profile $L$. 


\subsubsection{Fixed Points}

We now study FLR effects on the position and stability of the fixed points of the GSNM, $I^{*}=I_{n+1}=I_{n}$ and $\theta^{*}=\theta_{n+1}+m=\theta_{n}$, which, according to equations (2.29)(2.30), satisfy:

$$
\begin{aligned}
0 & =b J_{0}(\hat{\rho}) \sin \left(2 \pi \theta^{*}\right), \\
m & =a\left[\left(1-\frac{\bar{\rho}^{2}}{2}\right)-I^{* 2}\right],
\end{aligned}
$$

where $m$ is an integer number. For each $m \in \mathbb{Z}, a \neq 0, b J_{0}(\hat{\rho}) \neq 0$, and $\bar{\rho} \leq \sqrt{2\left(1-\frac{m}{a}\right)}$, there are four fixed points:

$$
\begin{array}{r}
P_{ \pm}=\left(0, \pm I_{*}(\bar{\rho})\right), \\
Q_{ \pm}=\left(\frac{1}{2}, \pm I_{*}(\bar{\rho})\right),
\end{array}
$$

where:

$$
I_{*}(\bar{\rho})=\frac{1}{\sqrt{2}} \sqrt{2\left(1-\frac{m}{a}\right)-\bar{\rho}^{2}} .
$$

The $\theta$ coordinate of $P_{ \pm}$and $Q_{ \pm}$does not depend on any parameters. For fixed $m$ and $a$, the $I$-coordinate depends only on $\bar{\rho}$ and can be determined by using the function (2.38). If $\bar{\rho}=\sqrt{2\left(1-\frac{m}{a}\right)}$, the pair of points $\left\{P_{+}, P_{-}\right\}$collide at $(0,0)$, and $\left\{Q_{+}, Q_{-}\right\}$collide at $\left(\frac{1}{2}, 0\right)$. Figure 2.3 shows the $I$-axis position of both pairs for $m=0$ and increasing $\bar{\rho}$. The collision occurs for $\bar{\rho}=\sqrt{2}$. For higher values of $\bar{\rho}$, the fixed points don't exist.

The stability of a $k$-periodic orbit of a map $M$ is determined by the residue:

$$
R=\frac{1}{4}\left[2-\operatorname{Tr}\left(\prod_{i=0}^{k-1} J\left(\vec{x}_{i}\right)\right)\right]
$$

where $J$ is the Jacobian matrix of $M$, evaluated at all points of the $k$-period orbit $\left\{\vec{x}_{i}\right\}_{i=0}^{k-1}$. If $0<R<1$, the periodic orbit is elliptic (stable); if $R<0$ or $R>1$, it is hyperbolic (unstable); and, finally, it is parabolic in the transition from one case to the other, which occurs for $R=0$ or $R=1$.

Applying formula (2.39) to the GNSM fixed points (2.36)-(2.37), we have:

$$
R\left(P_{ \pm}\right)=R\left(Q_{\mp}\right)=\mp \pi a b J_{0}(\hat{\rho}) I_{*}(\bar{\rho})
$$




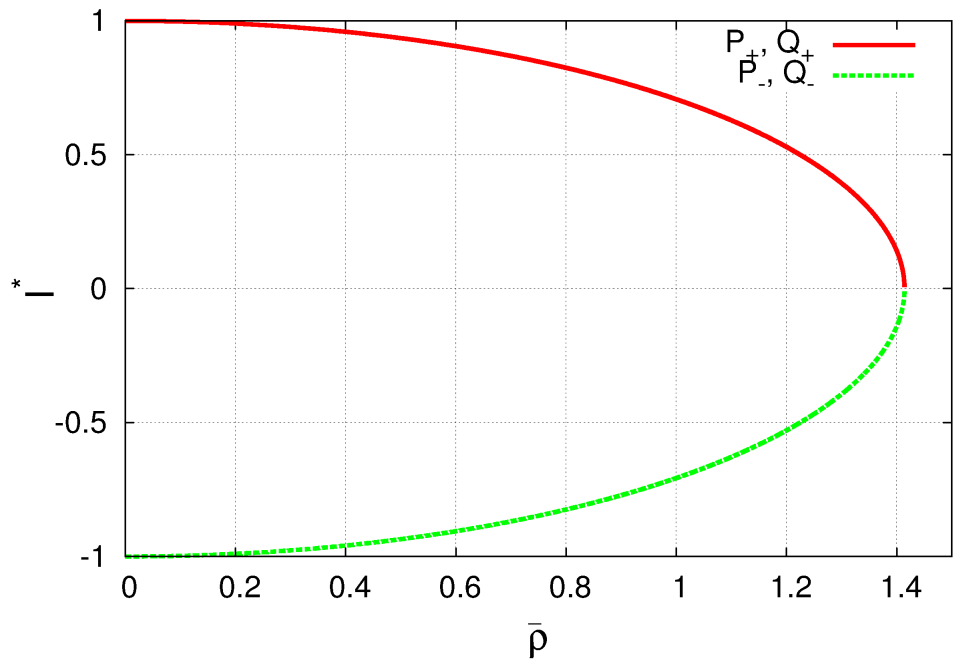

Figure 2.3: Coordinate $I$ of fixed points for $\mathrm{m}=0$ and increasing $\bar{\rho}$. The fixed points collide for $\bar{\rho}=\sqrt{2}$ and cease to exist for higher values of $\bar{\rho}$.

The stability of the fixed points $\left\{P_{ \pm}, Q_{ \pm}\right\}$with $m=0$ can be analyzed using the parameter $\Lambda(a, b, \hat{\rho}, \bar{\rho})=R\left(P_{-}\right)$. As shown in Fig. 2.4, depending on the value of $\Lambda$, there are three possible configurations. The symbol " $\mathrm{x}$ " denotes an hyperbolic point, and " $\mathrm{o}$ " an elliptic point. The points have their stability inverted when $-1<\Lambda<0$ (configuration $I I$ ), as indicated in figure 2.4b. All the fixed points are hyperbolic in configuration $I I I$ (fig. 2.4c), which occurs for $\Lambda<-1$ or $\Lambda>1$.

\subsubsection{Separatrix reconnection}

The location and stability of the fixed points determine the different phase space topologies of the GSNM. These topologies, illustrated in Figs. (2.5a)-(2.5c), are characteristic of nontwist maps and are called heteroclinic, separatrix reconnection, and homoclinic [19]. Since the Larmor radius changes the stability of the fixed points, it is expected that it will also change the topology. Figures 2.5(a)-(c) show the heteroclinic-type, separatrix reconnection, and the homoclinic-type topologies in Poincaré sections of the GSNM. We adopt different values of $a$ and $b$, and keep $\hat{\rho}$ and $\bar{\rho}$ fixed.

To determine the condition for separatrix reconnection associated to the fixed points $P_{ \pm}$and $Q_{ \pm}$with $m=0$, we follow $[?, 19]$ and approximate the GSNM in the vicinity of 


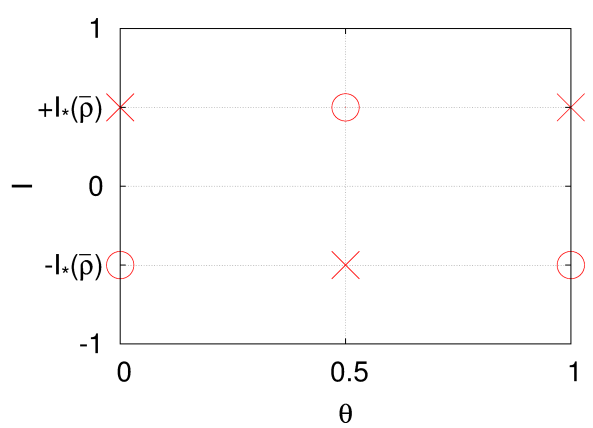

(a) Configuration I: $0<\Lambda<1$

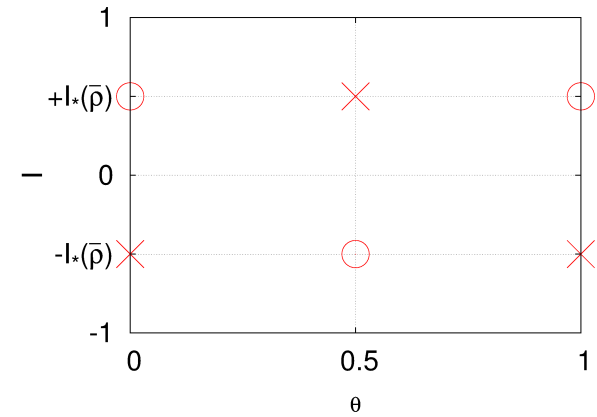

(b) Configuration II: $-1<\Lambda<0$

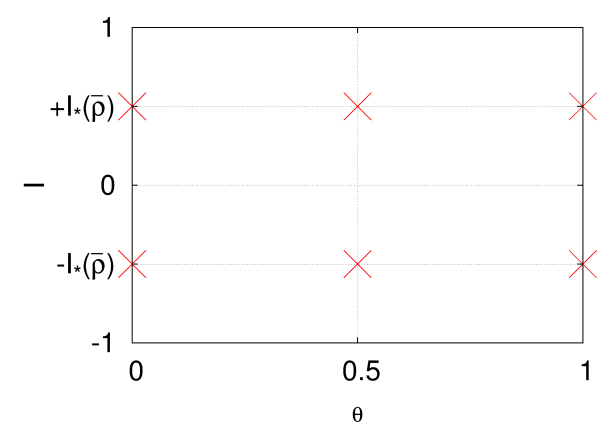

(c) Configuration III: $\Lambda<-1$ or $\Lambda>1$.

Figure 2.4: Stability of the fixed points points $P_{ \pm}$and $Q_{ \pm}$with $m=0$.

the fixed points by the Hamiltonian:

$$
H(I, \theta)=a\left[I\left(1-\frac{\bar{\rho}^{2}}{2}\right)-\frac{I^{3}}{3}\right]-\frac{b}{2 \pi} J_{0}(\hat{\rho}) \cos (2 \pi \theta),
$$

For $0<\Lambda<1$ (configuration I), separatrix reconnection occurs when $H\left(P_{+}\right)=H\left(Q_{-}\right)$. If $-1<\Lambda<0$ (configuration II), reconnection is observed when $H\left(P_{-}\right)=H\left(Q_{+}\right)$. Combining these two conditions, we obtain the reconnection line:

$$
a=\frac{3}{4 \pi} \sigma(\hat{\rho}, \bar{\rho}) b
$$

with slope:

$$
\sigma(\hat{\rho}, \bar{\rho})=\frac{\left|J_{0}(\hat{\rho})\right|}{\left(1-\frac{\bar{\rho}^{2}}{2}\right)^{\frac{3}{2}}} .
$$

The reconnection line divides the $a-b$ parameter space in two regions: one with heteroclinic-type topology $\left(\frac{a}{b}>\frac{3}{4 \pi} \sigma(\hat{\rho}, \bar{\rho})\right)$ and another with homoclinic-type topology $\left(\frac{a}{b}<\frac{3}{4 \pi} \sigma(\hat{\rho}, \bar{\rho})\right)$. The slope of the reconnection line is defined by the angle $\arctan \left[\frac{3}{4 \pi} \sigma(\hat{\rho}, \bar{\rho})\right]$, which varies according to the formula (2.43). Figure 2.6 shows isolines of the angle 


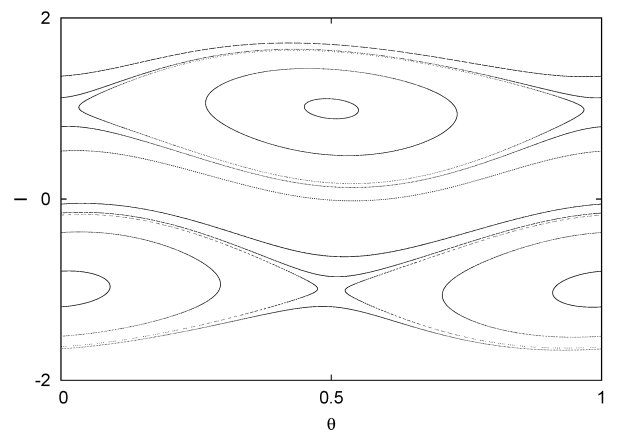

(a) Heteroclinic-type topology with $a=$ 0.0796 and $b=0.125$.

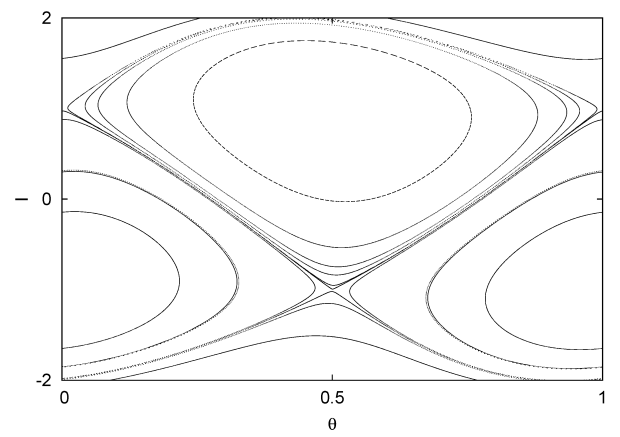

(b) Separatrix reconnection with $a=0.0478$ and $b=0.2$.

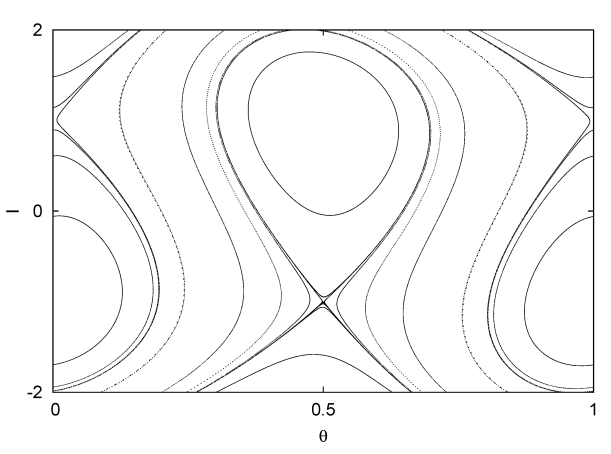

(c) Homoclinic-type topology with $a=$ 0.0239 and $b=0.3$.

Figure 2.5: GSNM phase space topologies associated with the fixed points $P_{ \pm}$and $Q_{ \pm}$with $m=0$. In all figures, the Larmor radius is such that $\hat{\rho}=0.05$ and $\bar{\rho}=0.01$. 
$\arctan \left[\frac{3}{4 \pi} \sigma(\hat{\rho}, \bar{\rho})\right]$. Keeping $a$ and $b$ fixed, the topology of the phase space remains unchanged if the parameters $\hat{\rho}$ and $\bar{\rho}$ vary over an isoline. As an example, consider the case for fixed $a$ and $b$ such that $a=\frac{3}{4 \pi} b$, which correponds to the reconnection condition in the standard nontwist map [19]. In this case, there is always a reconnection for any values of $\hat{\rho}$ and $\bar{\rho}$ over the red isoline of Fig. 2.6. The red isoline crosses the point $\hat{\rho}=\bar{\rho}=0$, at which the GNSM reduces to the standard nontwist map. For high (red) values of $\sigma$, the slope of the reconnection line approaches $\frac{\pi}{2}$, and the phase space is characterized by the homoclinic-type topology for a fixed $a$ and almost every value of the parameter $b$. For low (blue) values of $\sigma$, the slope of the reconnection line tends to 0 , and the phase space is characterized by the heteroclinic-type topology for a fixed $b$ and almost every value of the parameter $a$.

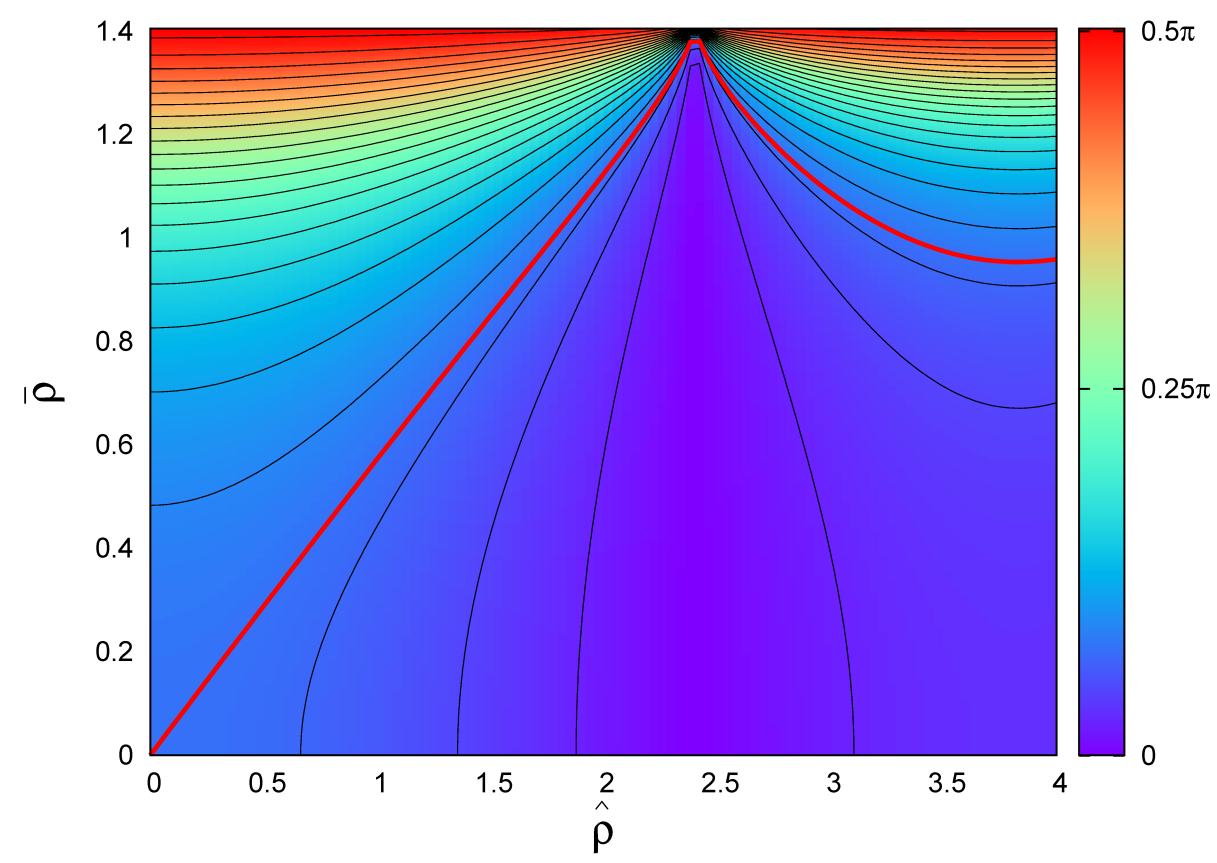

Figure 2.6: Isolines of $\arctan \left[\frac{3}{4 \pi} \sigma(\hat{\rho}, \bar{\rho})\right]$ : varying $\hat{\rho}$ and $\bar{\rho}$ along isolines does not change the topology of the phase space. For high (red) values of $\sigma$, homoclinic-type topology is observed for a fixed $a$ and almost every value of $b$. For low (blue) values of $\sigma$, heteroclinic-type topology is observed for a fixed $b$ and almost every value of $a$. 


\subsubsection{Nontwist transport barrier}

The transition to global chaos in the GSNM occurs with the destruction of the nontwist transport barrier (NTB). One of main properties of nontwist 3/2-degrees of freedom Hamiltonian systems, the NTB is a robust non-chaotic region of spanning KAM curves dividing the phase space. The NTB is robust in the sense that their spanning KAM curves are more resistant to perturbation than the other spanning KAM curves in the system. NTBs are observed in both continuous in time Hamiltonian systems, and time discrete area preserving models. The GSNM is an example of such a discrete model.

Let us consider the following form of Hamiltonian systems with $3 / 2$ degrees of freedom corresponding to a time-dependent perturbation of a one degree of freedom Hamiltonian:

$$
H=H_{0}(I)+\epsilon V(I, \theta, t)
$$

where $I$ and $\theta$ are the action-angle variables of the integrable system, $H_{0}$, and the parameter $\epsilon$ controls the strength of the time-periodic pertubative term $V$. In this case, the twist or non-degenerate condition is given by:

$$
\frac{d \Omega}{d I}=\frac{d^{2} H_{0}}{d I^{2}} \neq 0
$$

for all $I$. If there is at least one critical or degenerate point in the frequency profile, i.e. points such that $d \Omega / d I=0$ for $I=I_{c}$, the non-degenerate condition is violated, and the system defined by (2.44) is called as a nontwist or degenerate 3/2-degrees of freedom Hamiltonian system.

One of the main properties of such systems is the presence of NTBs, one for each degenerate point of the frequency profile. This property was described originally in Ref. [19] and was called banded chaos. A more recent work referred to the same property as strong Kolmogorov-Arnold-Moser stability [23]. In this case, the phase space can be almost completely occupied by chaotic orbits, but robust spanning KAM curves forming constitute the NTB can still be present.

Figure 2.7 shows a GSNM Poincaré section with a NTB. The Poincaré section presented in figure 2.7 is the same for any values of $b$ and $\hat{\rho}$ such that: $a=0.354, \bar{\rho}=0$, and $b J_{0}(\hat{\rho})=0.8$. We see that the phase space is characterized predominantly by chaotic 


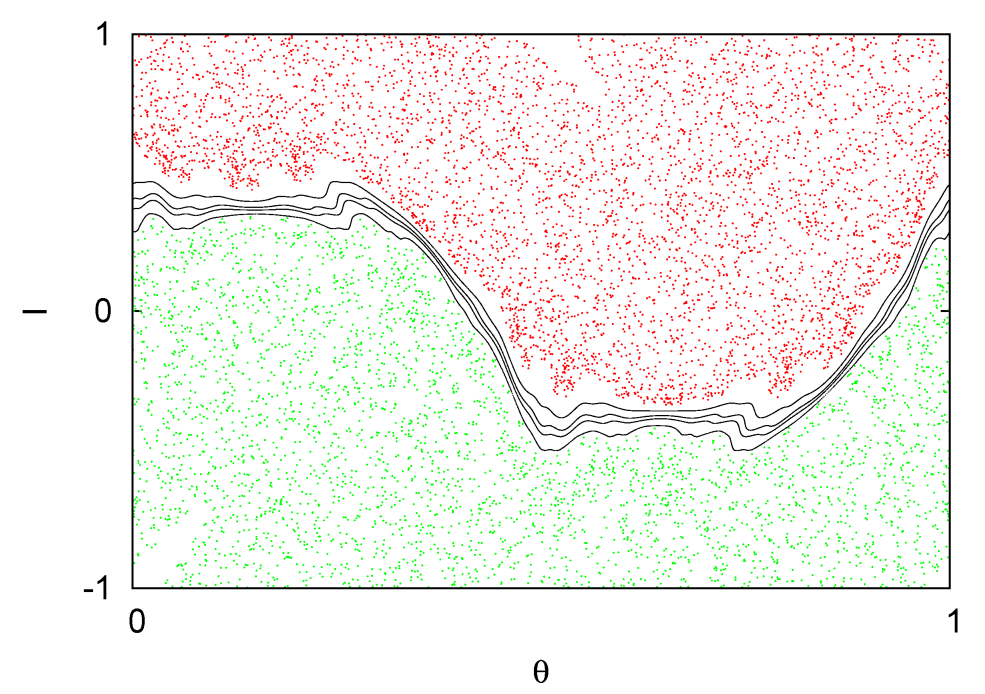

Figure 2.7: Nontwist transport barrier in the GSNM: robust region of spanning KAM curves (black curves) splitting the phase space in two chaotic regions, indicated by the red and green orbits.

orbits (green and red regions), but it has a robust "belt" of spanning KAM curves (black curves). As we have already seen, the GSNM can be obtained by integrating the equations of motion of the 3/2-degrees of freedom Hamiltonian (2.8) with a frequency defined by (2.25). The Hamiltonian system is degenerate because its frequency has a critical point (a maximum located at $x=0$ ).

\subsubsection{Breakup Diagrams}

Here we study the transition to global chaos in the GSNM, which is related to the destruction of the robust spanning KAM curves that constitute the NTB. It is important to remark that the transition to global chaos in nontwist systems is still an open problem. However, a possible approach to this problem consists of estimating the parameter values, or the critical thresholds, that determine the breakup of the shearless KAM curve. We say that a KAM curve is destroyed or broken when it becomes chaotic by changing parameter values.

The shearless curve, in absence of perturbation, is the KAM curve located at the value of action variable that violates the twist condition and defines a degenerate point in the frequency's profile. For this reason, according to the "strong KAM stability" argument 
developed in [23], the shearless curve corresponds to a "degenerate tori", which has the same robustness properties that characterizes the NTB's spanning KAM curves. Thus, we can say that the shearless curve belongs to the NTB, and the parameters values related to the NTB destruction can be estimated from the parameter values that determine the breakup of shearless curve.

A standard way to determine the parameter values of shearless curve's breakup is based on the indicator point (IP) method (see, for example, Refs. [6,21,38,40]). This method consists on finding the parameter values for which the iterations of the indicator point, which defines the indicator point orbit (IPO), are chaotic. If the IPO is quasiperiodic, it covers a shearless KAM curve. When the IPO is chaotic, the shearless curve is broken. The indicator points can be found in nontwist maps with special symmetries by computing the fixed points of transformations of the reversing symmetry group. Further details can be found in Ref. [39].

Using the same arguments and a procedure similar to the one in [40], which shows how to obtain the IPs for the standard nontwist map (see Appendix B of Ref. [40] and also Appendix B at the end of this work for more details), we obtain the following IPs for the gyro-averaged standard nontwist map:

$$
\begin{gathered}
z_{0}^{1}=\left(\frac{1}{4},+\frac{b J_{0}(\hat{\rho})}{2}\right), \quad z_{0}^{2}=\left(\frac{3}{4},-\frac{b J_{0}(\hat{\rho})}{2}\right), \\
z_{1}^{1}=\left(\frac{a}{2}\left(1-\frac{\bar{\rho}^{2}}{2}\right)+\frac{1}{4} \bmod 1, \quad 0\right), \\
z_{1}^{2}=\left(\frac{a}{2}\left(1-\frac{\bar{\rho}^{2}}{2}\right)+\frac{3}{4} \bmod 1,0\right) .
\end{gathered}
$$

Once the IPs are found, the next step is to obtain the critical thresholds using an appropriate criterion to determine if the IPO is chaotic. Our criterion is based on the technique proposed in [28], which considers the recurrence properties of the IPO in conjunction with Slater's Theorem [29]. Recurrence properties and their relation to the Slater's Theorem were also discussed in [30]. In Ref. [31], the technique described in [28] was used to study the breakup of the shearless curve in the nontwist standard map.

The Slater's Theorem states that, for any quasiperiodic motion on the circle, there are at most three different recurrences, or return times, in any connected interval. Although the Slater's Theorem was originally formulated for circle maps, it can also be applied to 
two-dimensional systems, once the dynamics on a KAM curve is mapped to a quasiperiodic rotation on a circle [28]. The recurrence time is defined as the number of iterations that an orbit takes to return to a neighbourhood of a point. Our procedure to characterize the dynamics of an indicator point orbit of the GSNM is the following: with any one of the indicator points as the initial condition, we compute the orbit for a number $N$ of iterations and choose the point of the orbit which has the maximum number of different recurrence times. If there are more than three different recurrence times, we conclude that the orbit is chaotic and the corresponding KAM curve is destroyed.

More precisely, the procedure consists of the following steps:

- Compute the orbit $O=\left\{u_{k}\right\}_{k=0}^{N}$ with initial condition $u_{0}=z_{1}^{1}$, where $N$ is the number of iterations.

- Construct the recurrence matrix ${ }^{2}$ :

$$
R_{i j}=\Theta\left(\epsilon-\left\|u_{i}-u_{j}\right\|\right),
$$

where $i, j \in\{0, \ldots, N\}, u_{i}, u_{j} \in O, \Theta$ is the Heaviside function, and $\epsilon$ is a parameter defining the size of the neighbourhood. If the distance beetween $u_{i}$ and $u_{j}$, given by the norm $\left\|u_{i}-u_{j}\right\|$, is less than $\epsilon, R_{i j}=1$; otherwise, $R_{i j}=0$.

- Define the recurrence time $\tau_{i j}$ as $\tau_{i j}=|i-j|$ such that $i \neq j$ and $R_{i j}=1$. That is, there is a recurrence time $\tau_{i j}$ if the orbit $O$ crosses the neighbouhood of $u_{j}$ at the $i$-th iteration. For each point $u_{j}$, compute the set of recurrences $S_{R}^{(j)}=\cup_{i=0, i \neq j}^{N}\left\{\tau_{i j}\right\}$. Note that just different recurrence times belong to $S_{R}^{(j)}$

- Determine the maximum number of different recurrence times:

$$
n_{R}=\max \left\{n\left(S_{R}^{(j)}\right)\right\}_{j=0}^{N}
$$

where $n$ is the number of recurrence times belonging to $S_{R}^{(j)}$, and use the Slater's Theorem to conclude that the orbit $O$ is chaotic if $n_{R}>3$.

\footnotetext{
${ }^{2}$ Here we borrow the definition presented in Ref. [30] of the binary matrix $R$. Its graphical representation, called recurrence plot, can be used to analyse the recurrence properties of any dynamical system.
} 
For $n_{R} \leq 3$, the characterization of the dynamics using the Slater's Theorem is inconclusive because the orbit might be periodic, quasiperiodic or even chaotic. In this case, we can increase the number of iterations, $N$, to explore if $n_{R}$ increases beyond 3 . If the condition $n_{R} \leq 3$ still persists and no change in $n_{R}$ is observed, we assume that the orbit is periodic for $n_{R}=1$ and quasiperiodic for $n_{R}=3$. The condition $n_{R}=2$ requires a more careful analysis with higher values $N$. Increasing $N, n_{R}$ stabilizes in 3 , indicating quasiperiodic dynamics, or assumes values greater than 3, which is the case for chaotic dynamics. Although more rigourous criteria for the case $n_{R} \leq 3$ are lacking, the numerical results of Refs. $[28,30]$ support the use of the recurrence properties and their relation to the Slater's Theorem as a computationally fast diagnostic to recognize chaotic motion.

We now apply this diagnostic to compute breakup diagrams for the GSNM showing regions in the parameter space where the shearless curve is broken. The diagrams were constructed by applying the procedure described above to IPOs generated with different values of the GSNM parameters. Each set of values corresponds to a point on 2-dimensional parameter space. Since the GSNM has four parameters, the breakup diagrams are constructed varying two parameters at a time while keeping the others constant. Our first examples are presented in Figs. 2.8a and 2.8b. In both diagrams, we used a grid of $1000 \times 1000$ points and vary $b$ and $\hat{\rho}$. We fix $\bar{\rho}=0$ (which means that $\rho \ll L$ ) and $a=0.1$. For each point on the grid, the IPO and its $n_{R}$ are computed for $\epsilon=0.1$. The color of each point is defined by the value of $n_{R}$. The relation between the colors and the $n_{R}$ values is indicated in the color palette. For example, blue corresponds to $n_{R}=1$; dark-blue, $n_{R}=2$; green, $n_{R}=3$; magenta, $n_{R}=4$; and all points with $n_{R} \geq 9$ are plotted in yellow.

To construct diagram 2.8a, we used $N=100$. Although the number of iterations is increased to $N=1000$ in diagram 2.8b, the main geometric features that enable us to distinguish between regular and chaotic dynamics are mantained. The main difference is observed in points with $n_{R}=2$ (dark-blue points). Increasing the number of iterations, most of these points have their color changed corresponding to $n_{R}>3$. Diagram 2.8a shows that, even for a low number of iterations, it's possible to identify the breakup of the shearless curve in domains where $n_{R}>3$. These domains have points plotted with 


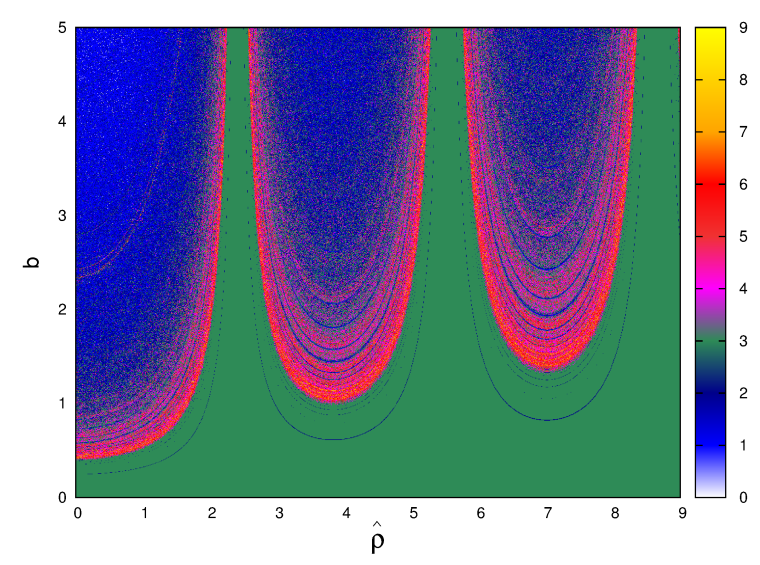

(a) $N=100$

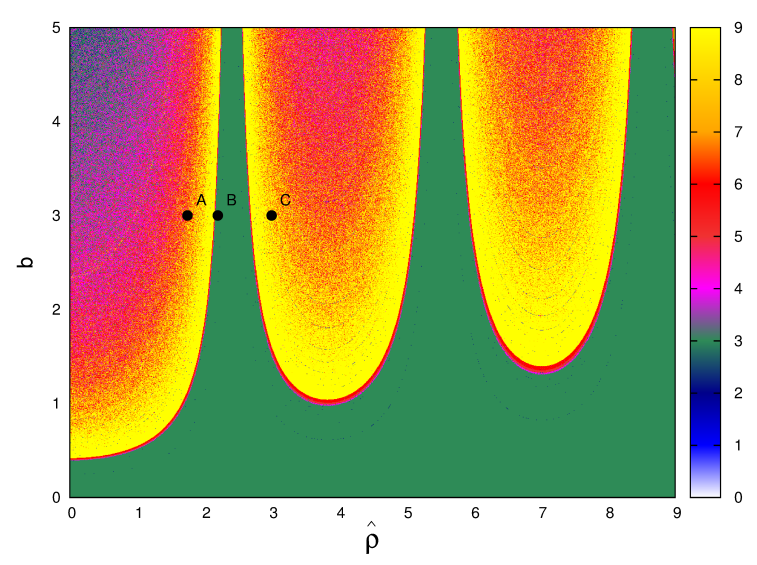

(b) $N=1000$

Figure 2.8: GSNM breakup diagrams for $\hat{\rho}$ versus $b$, with $\bar{\rho}=0$, and $a=0.1$. For each point $(\hat{\rho}, b)$ in the diagram, the IPO and the correspondent $n_{R}$ are computed with $\epsilon=0.1$. The color pallete indicates the value of $n_{R}$. The breakup of the shearless curve can be detected in the points where $n_{R}>3$. The IPO is quasiperiodic in the green region $\left(n_{R}=3\right)$ which corresponds to low effective perturbation $b_{e f}$, i.e, low $b$ or $\hat{\rho}$ near a zero of $J_{0}$.

magenta, red, and yellow colors. Green points $\left(n_{R}=3\right)$ are concentrated in domains with low effective perturbation $b_{e f}$, which means a low perturbation $b$ or $\hat{\rho}$ close to a zero of the zero-order Bessel function. As verified in Figs. 2.9(a)-(c), $n_{R}=3$ (green points) corresponds to quasiperiodic IPOs. For points outside the green region (e.g. point $A$ in Fig. 2.8b), the IPO is chaotic, and, as shown in Fig. 2.9a, the shearless curve is destroyed. Changing $\hat{\rho}$ to a value near a zero of $J_{0}$ such that the point moves inside the green region (e.g. point $B$ of Fig. 2.8b), the shearless KAM curve is restored because the IPO becomes quasi-periodic, as shown by the red orbit in Fig. 2.9b. Figure 2.9c shows that the shearless KAM curve is broken again for parameter values outside the green region (e.g. point $C$ in Fig. 2.8b).

Breakup diagrams with varying $a$ and $\hat{\rho}$ are shown in figures 2.10a and 2.10b. The number of iterations adopted to construct the first diagram was $N=100$. In the second diagram, we increased the number of iterations to $N=1000$. As before, the green points $\left(n_{R}=3\right)$, which are indicators of non-chaotic dynamics, are concentrated near the zeros of $J_{0}$. A high occurrence of points with $n_{R}=3$ is also observed in regions with low $a$. When the number of iterations is increased from $N=100$ to $N=1000$, we can see that 


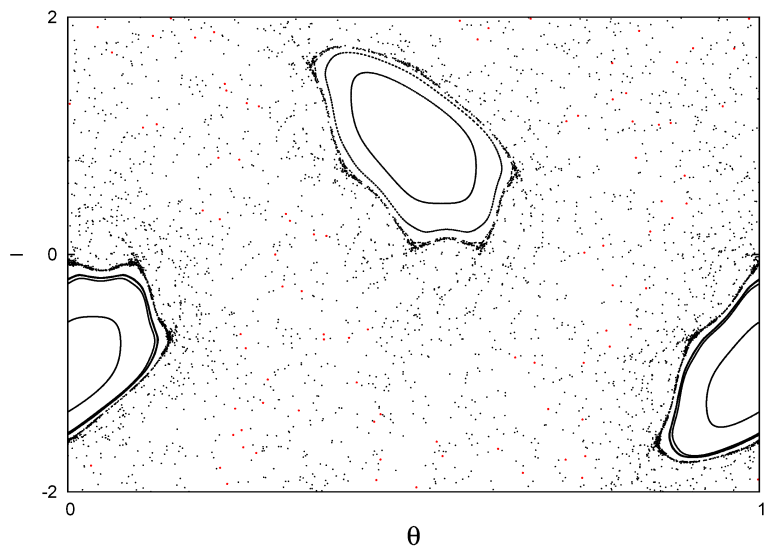

(a) $\hat{\rho}=1.75$

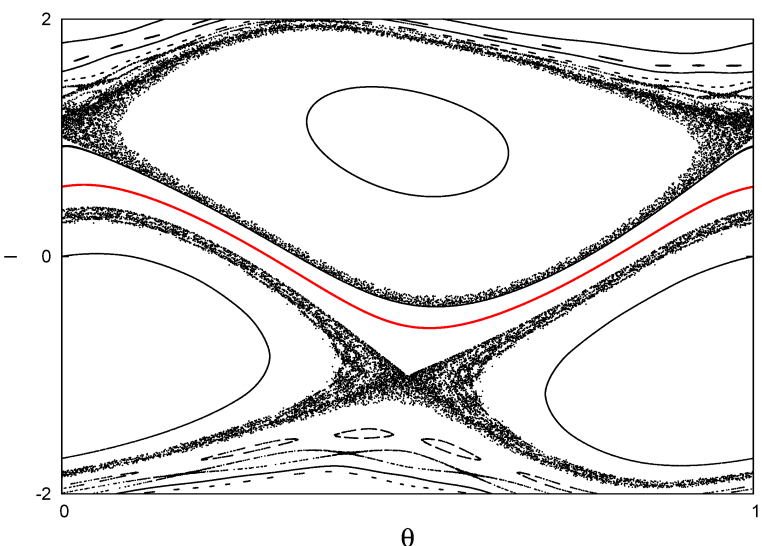

(b) $\hat{\rho}=2.2$

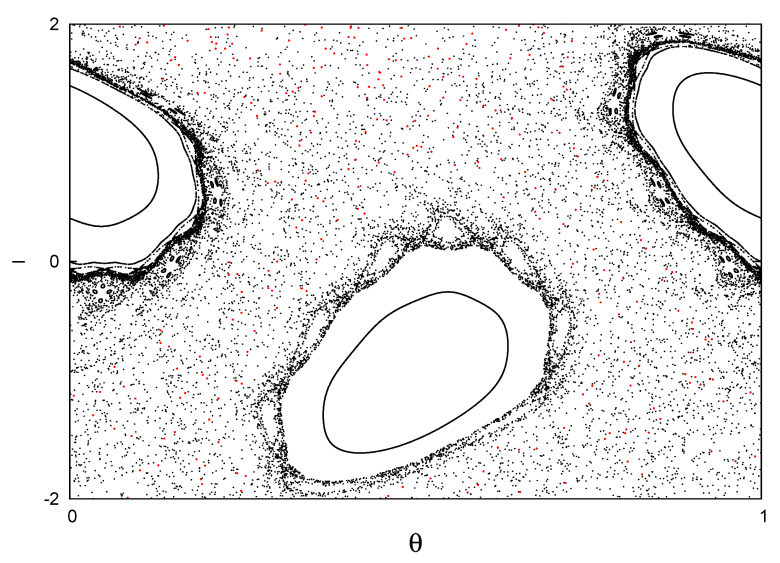

(c) $\hat{\rho}=3$

Figure 2.9: The shearless KAM curve, identified through a quasiperiodic IPO (red orbit), is restored near the zeros of $J_{0}$. Fixed parameters: $a=0.1 ; b=3 ; \bar{\rho}=0$. (a) For $\hat{\rho}=1.75$ (point A, figure 2.8b), the IPO is chaotic. (b) For $\hat{\rho}=2.2$ (point $\mathrm{B}$ ), the IPO (red orbit) is quasiperiodic. (c) For $\hat{\rho}=3$ (point $\mathrm{C}$ ), the shearless KAM curve is broken again. 


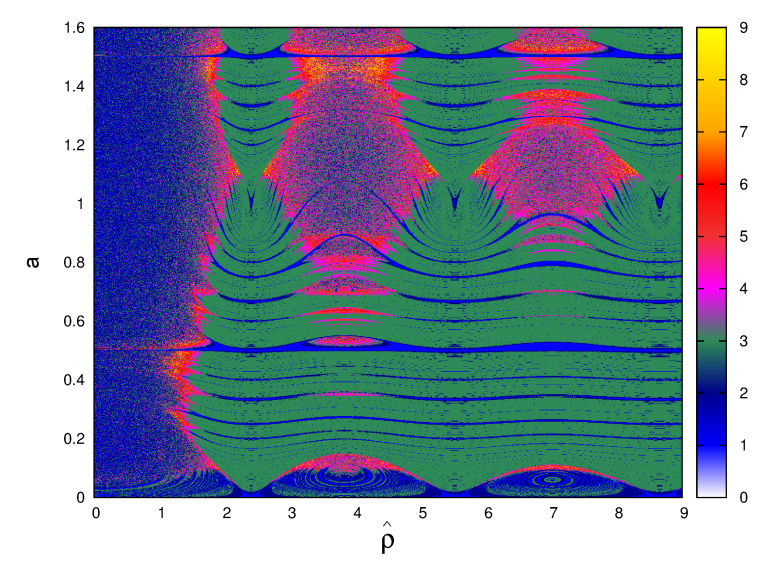

(a) $N=100$

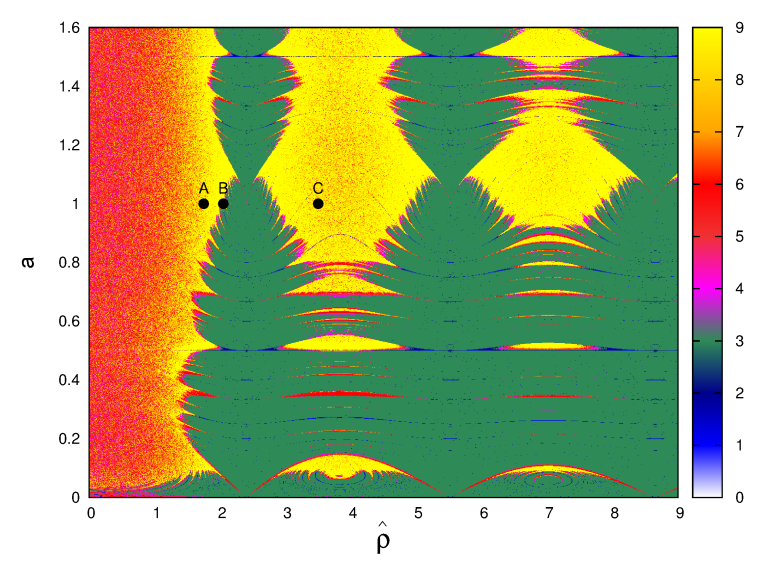

(b) $N=1000$

Figure 2.10: GSNM breakup diagrams for $\hat{\rho}$ versus $a$, with $\bar{\rho}=0$ and $b=1.5$. Neighbouhood size: $\epsilon=0.1$. Green points, corresponding to quasiperiodic dynamics, are concentrated near the zeros of $J_{0}$ and in domains with low $a$.

the main geometric features remain the same, although many of the blue points $\left(n_{R}=1\right.$ or $n_{R}=2$ ) have their colors changed such that $n_{R}>3$. Poincaré sections for parameters corresponding to points $A, B$, and $C$ in Fig. 2.10b are shown in figures 2.11a-c. As expected, the shearless KAM curve is broken at points $A$ and $C$, and restored at point $B$.

Additional examples of breakup diagrams are presented in figures 2.12a-d. The parameters are the same as those in Figs. $2.8 \mathrm{~b}$ and $2.10 \mathrm{~b}$, but the value of the fixed parameter $\bar{\rho}$ is higher. Comparing figures $2.12 \mathrm{a}-\mathrm{b}$ to $2.8 \mathrm{~b}$, we see that increasing $\bar{\rho}$ increases the number of green points $\left(n_{R}=3\right)$ and the robustness of the shearless KAM curve. That is, higher values of the perturbation parameter $b$ are required to make the IPO chaotic. As shown in Figs. 2.12c-d, the distribution of points with $n_{R}=3$ is changed with the increasing of $\bar{\rho}$, resulting in different critical thresholds but with no significant suppression of green points. Red and yellow points disappear in certain regions and reappear in others, as can be seen by comparing Figs. 2.10b, 2.12c and 2.12d.

Summarizing: in this section we used a computational technique based on the recurrence properties of the IPO to estimate the critical parameter values for the breakup of the shearless KAM curve. In particular, we computed breakup diagrams to understand 


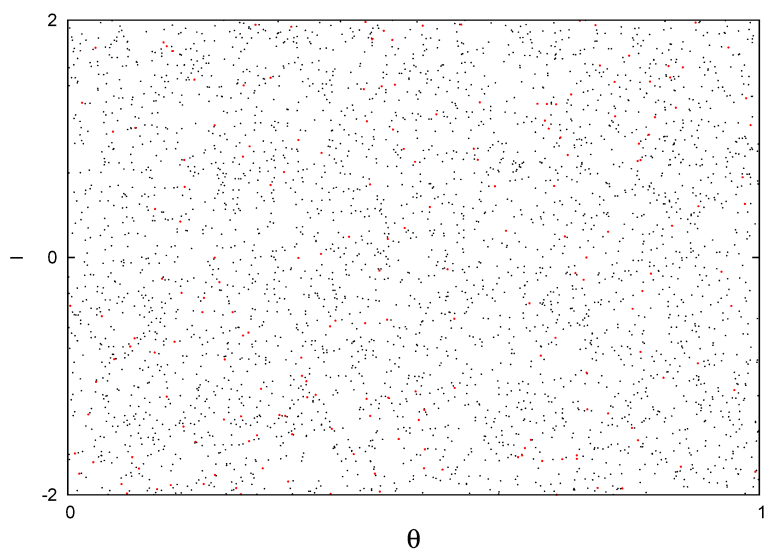

(a) $\hat{\rho}=1$ (point A)

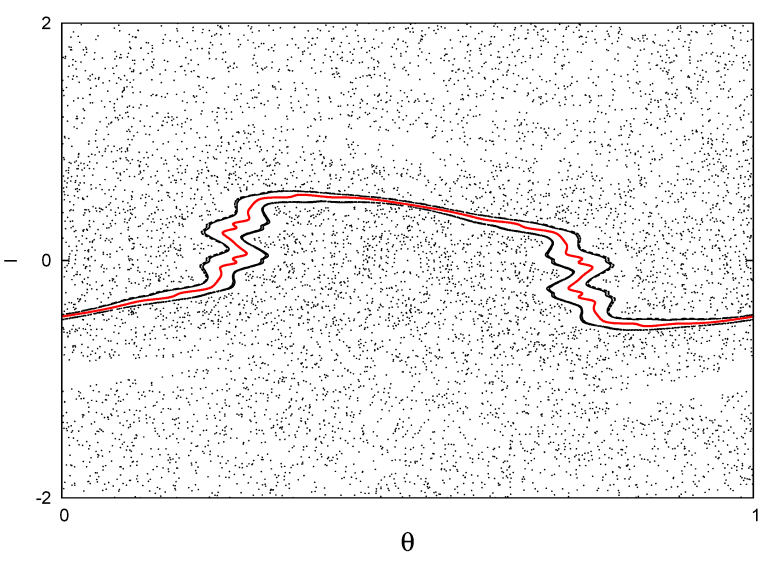

(b) $\hat{\rho}=2.05$ (point B)

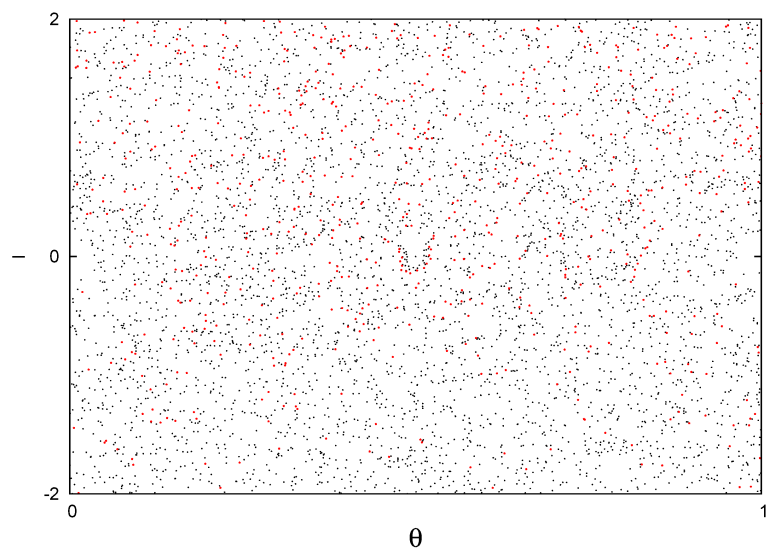

(c) $\hat{\rho}=3.5($ point C)

Figure 2.11: Poincare sections of the GSNM map for points $A, B$ and $C$, in the diagram 2.8b. The shearless KAM curve corresnponding to point $\mathrm{B}$ is present, but it breaks at $\mathrm{A}$ and $\mathrm{C}$. Fixed parameters: $a=1 ; b=1.5$; $\bar{\rho}=0$ 


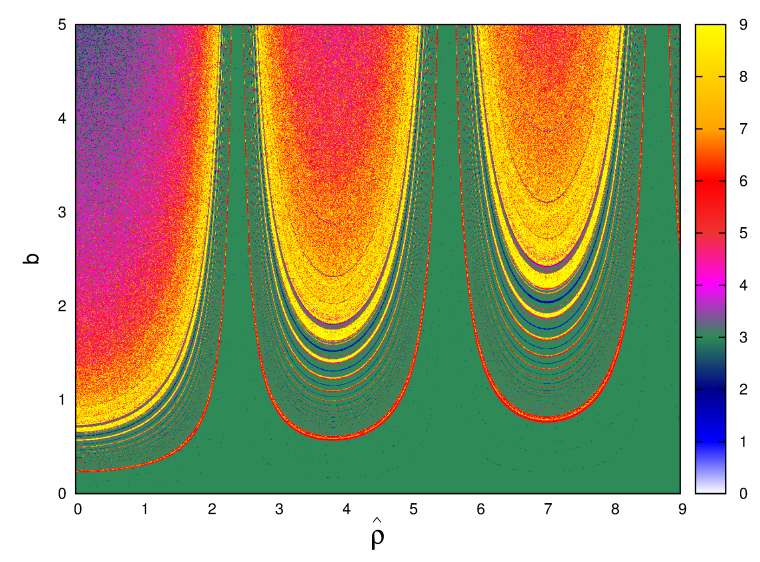

(a) $\bar{\rho}=0.8 ; a=0.1$

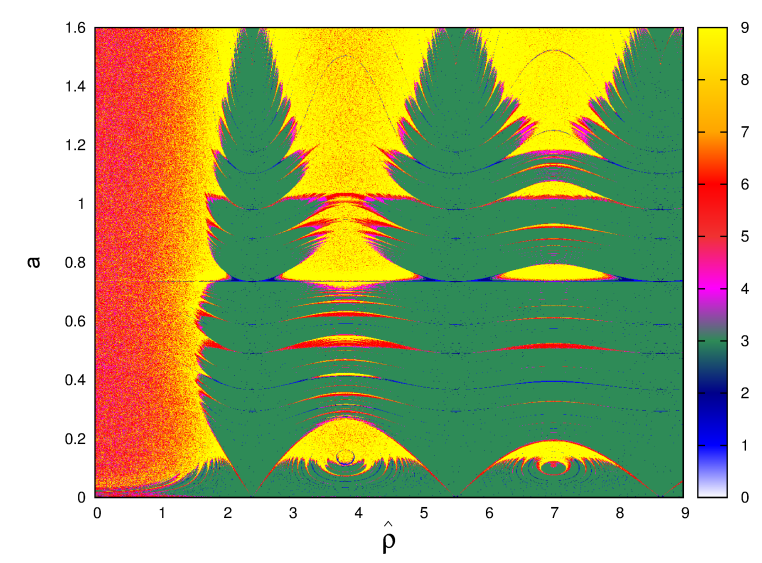

(c) $\bar{\rho}=0.8 ; b=1.5$

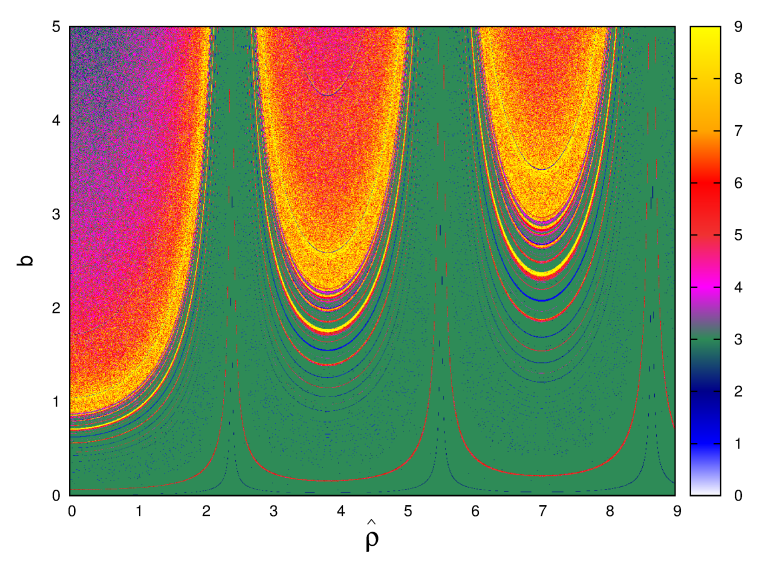

(b) $\bar{\rho}=1.2 ; a=0.1$

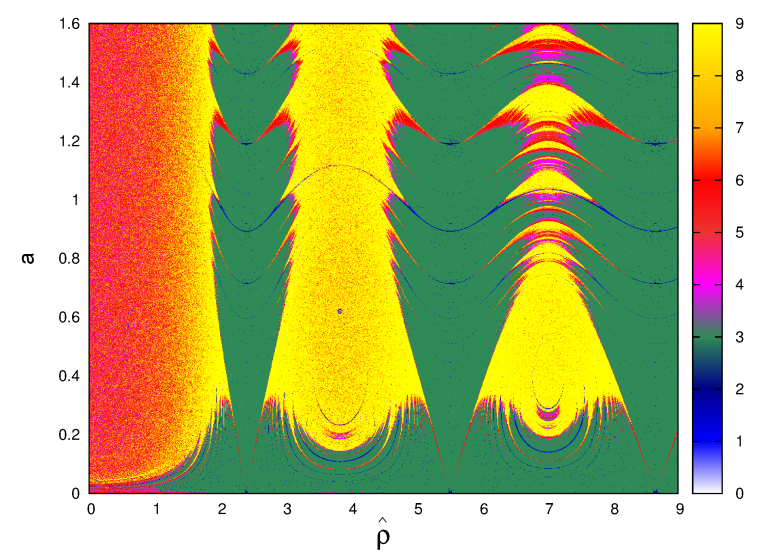

(d) $\bar{\rho}=1.2 ; b=1.5$

Figure 2.12: Diagram: (a) and (b): the robustness of the shearless KAM increases when $\bar{\rho}$ increases, which means that more green points $\left(n_{R}=3\right)$ are observed. Diagrams (c) and (d): the critical thresholds change when the value of $\bar{\rho}$ changes. For all diagrams, we adopted $N=1000$ and $\epsilon=0.1$. 
the role of the Larmor radius in the destruction and formation of shearless spanning KAM curves. FLR effects were studied by varying the parameters $\hat{\rho}$ or $\bar{\rho}$. When $\hat{\rho}$ is close to a zero of $J_{0}$, the shearless curve becomes more robust to perturbation in $b$. An explanation for this effect is that, no matter how high the values of $b$ are, the zeros of $J_{0}$ make the GSNM's Hamiltonian integrable, as seen in equation (2.8). As $\hat{\rho}$ approaches a zero of $J_{0}$, the shearless curve and the other spanning KAM curves that make up the nontwist transport barrier are restored. Another FLR effect is observed by changing the parameter $\bar{\rho}$. As can be seen in Figs. 2.12a-b, the robustness of the shearless curve and the critical thresholds can also be modified by increasing $\bar{\rho}$.

\subsection{Gyro-averaged quartic nontwist map (GQNM)}

In this section, we propose another area-preserving map, the gyro-averaged quartic nontwist map (GQNM). A key property of the GQNM is that it has a zonal flow bifurcation, similar to the one observed in the nontwist Hamiltonian system of Ref. [6]. The Hamiltonian system in [6] is a drift-wave model of the $\mathbf{E} \times \mathbf{B}$ transport with FLR effects, and the zonal flow bifurcation corresponds to a bifurcation of the maximum of the frequecy's profile that occurs when the Larmor radius increases.

As in the previous maps, the GQNM equations are obtained from the gyro-averaged drift wave map in equations (2.11) and (2.12). Here we adopt an equilibrium potential similar to the one proposed by [6]:

$$
\phi_{0}(x)=\alpha \tanh \left(\frac{x}{L}\right)
$$

where $\alpha$ and $L$ are dimensional constants. Applying the gyro-average operation (2.4) to (2.50), we have:

$$
\left\langle H_{0}(x)\right\rangle_{\varphi}=\frac{\alpha}{2 \pi B_{0}} \int_{0}^{2 \pi} \tanh \left(\frac{x}{L}+\frac{\rho}{L} \cos \varphi\right) d \varphi .
$$

The nonlinear frequency, according to (2.13), is defined by:

$$
\Omega(I)=\frac{\alpha}{2 \pi L B_{0}} \int_{0}^{2 \pi} \operatorname{sech}^{2}(I+\bar{\rho} \cos \varphi) d \varphi
$$

where $I=x / L$, and $\bar{\rho}=\rho / L$. The zonal flow bifurcation occurs due to a bifurcation of a critical point in the profile of function (2.52), which can be evaluated using numerical 


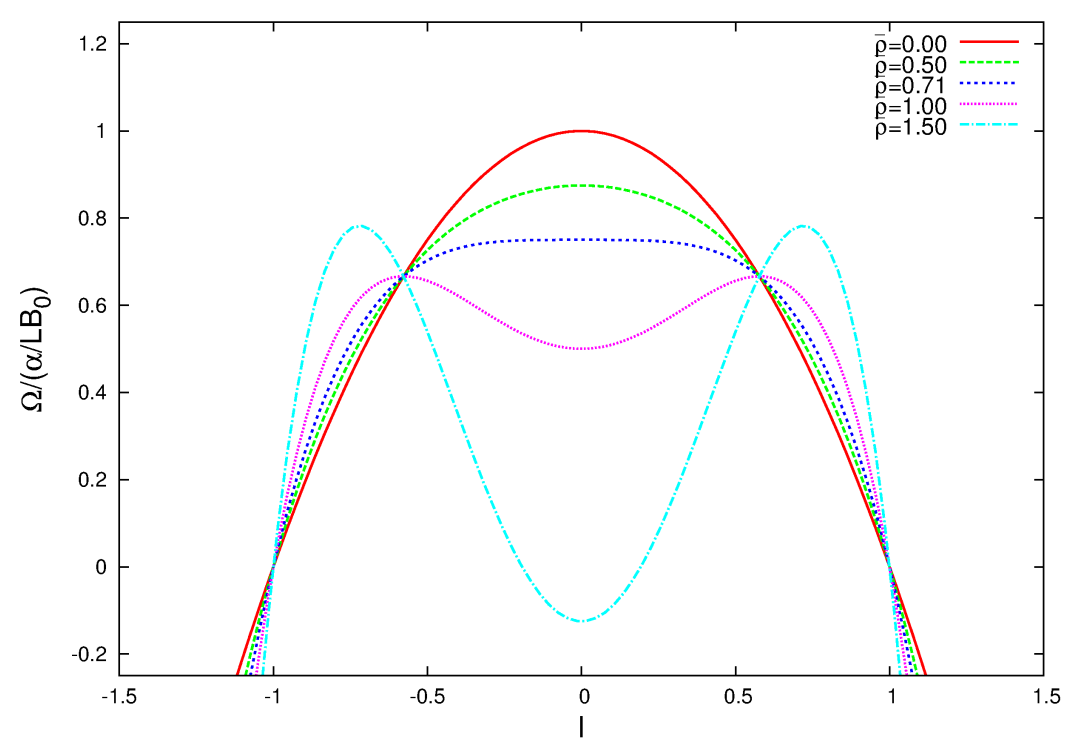

Figure 2.13: Bifurcation of the critical point in the frequency profile (2.53)

methods or approximated by Taylor expansions. To analyse, in a simple way, the FLR effect on the zonal flow in (2.52), we consider the following approximation:

$$
\Omega(I)=\frac{\alpha}{L B_{0}}\left(1-I^{2}\right)\left\{1-\bar{\rho}^{2}\left[\frac{3}{2}\left(1-I^{2}\right)-1\right]\right\}
$$

valid for small values of $|I|$ and $\bar{\rho}$. Figure 2.13 shows profiles of (2.53) for different values of $\bar{\rho}$. For $\bar{\rho}=0$, there is only one critical $\left(\Omega^{\prime}=0\right)$ maximum point, located at $I=0$. Increasing $\bar{\rho}$ leads to the increasing the profile's "flatness", followed by a critical point bifurcation, where the maximum becomes a minimum and two maxima appear. The bifurcation threshold $\bar{\rho}_{b}$ can be determined from the condition:

$$
\left.\frac{\partial^{2} \Omega}{\partial I^{2}}\right|_{I=0, \bar{\rho}=\bar{\rho}_{b}}=0 .
$$

The second-order derivative of (2.53) is given by:

$$
\left.\frac{\partial^{2} \Omega}{\partial I^{2}}\right|_{I=0}=-2+4 \bar{\rho}^{2} .
$$

From (2.54) and (2.55), it follows that the bifurcation threshold occurs at:

$$
\bar{\rho}_{b}=1 / \sqrt{2} \text {. }
$$

Let $I_{n}=x_{n} / L, \theta_{n}=k y_{n} / 2 \pi$, and $\hat{\rho}=k \rho$. Using (2.53), we get, from (2.11) and (2.12), 
the GQNM:

$$
\begin{aligned}
& I_{n+1}=I_{n}+b J_{0}(\hat{\rho}) \sin \left(2 \pi \theta_{n}\right) \\
& \theta_{n+1}=\theta_{n}+a\left(1-I_{n+1}^{2}\right)\left\{1-\bar{\rho}^{2}\left[\frac{3}{2}\left(1-I_{n+1}^{2}\right)-1\right]\right\}, \bmod 1
\end{aligned}
$$

where $a=k \alpha / \omega_{0} L B_{0}$, and $b$, the perturbation parameter, is given by $b=2 \pi a A / \alpha$. As in the previous maps, an effective perturbation parameter can be defined as $b_{e f}=b J_{0}(\hat{\rho})$. Like the GSNM, the GQNM reduces to the standard nontwist map for $\hat{\rho}=\bar{\rho}=0$. In what follows, we discuss FLR effects on the GQNM's fixed points and nontwist transport barriers.

\subsubsection{Fixed Points and nontwist transport barriers}

In a similar way as we have done for the GSNM (section 2.3.1), FLR effects on the location and stability of period-1 orbits can be analysed in the GQNM. The fixed points $\left(\theta^{*}, I^{*}\right)$ of the GQNM satisfy:

$$
\begin{aligned}
0 & =b J_{0}(\hat{\rho}) \sin \left(2 \pi \theta^{*}\right) \\
m & =a\left(1-I^{* 2}\right)\left\{1-\bar{\rho}^{2}\left[\frac{3}{2}\left(1-I^{* 2}\right)-1\right]\right\}, \quad m \in \mathbb{Z}
\end{aligned}
$$

For $m=0$, which corresponds to the simplest case, $a \neq 0$, and $b J_{0}(\hat{\rho}) \neq 0$, there are four fixed points, given by:

$$
P_{1}^{ \pm}=(0, \pm 1), \quad Q_{1}^{ \pm}=\left(\frac{1}{2}, \pm 1\right)
$$

If $\bar{\rho} \geq 2 \bar{\rho}_{b}=\sqrt{2}$, there is an adittional set of fixed points:

$$
P_{2}^{ \pm}=(0, \pm h(\bar{\rho})) \quad Q_{2}^{ \pm}=\left(\frac{1}{2}, \pm h(\bar{\rho})\right)
$$

where the function $h(\bar{\rho})$ is defined by:

$$
h(\bar{\rho})=\sqrt{\frac{\left(\bar{\rho}^{2}-2\right)}{3 \bar{\rho}^{2}}}
$$

Figure 2.14 shows the $I$ coordinates of the fixed points with $m=0 . P_{1}^{ \pm}$and $Q_{1}^{ \pm}$exist for all $\bar{\rho} \geq 0$ and are always located at $I= \pm 1 . P_{2}^{ \pm}$and $Q_{2}^{ \pm}$only exist for $\bar{\rho} \geq 2 \bar{\rho}_{b}=\sqrt{2}$ 


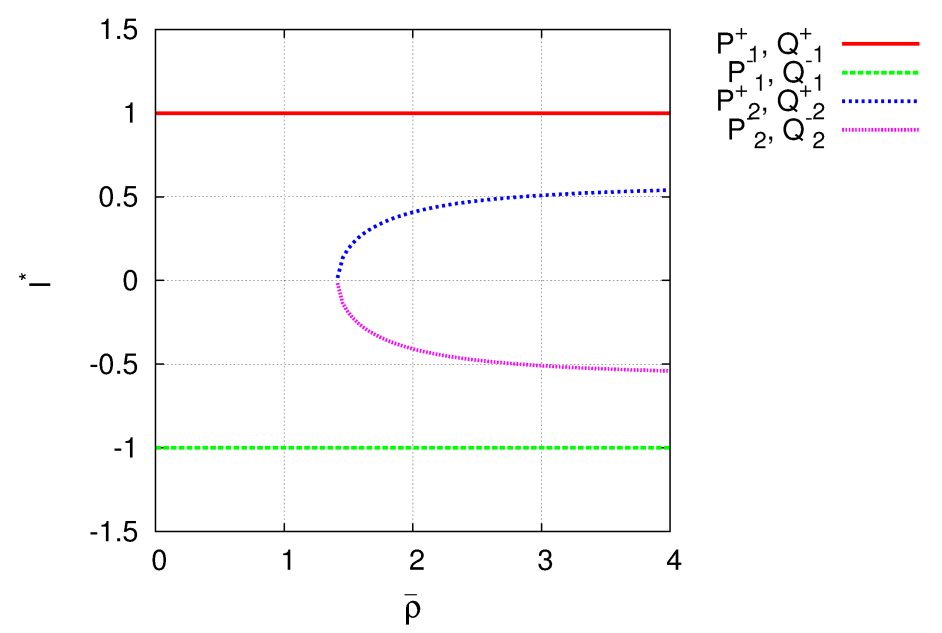

Figure 2.14: $I$ coordinates of fixed points with $m=0$. The set $\left\{P_{1}^{ \pm}, Q_{1}^{ \pm}\right\}$is fixed in the $I$-axis and exists for any $\bar{\rho} \geq 0$. The set $\left\{P_{2}^{ \pm}, Q_{2}^{ \pm}\right\}$only exists for $\bar{\rho} \geq 2 \bar{\rho}_{b}=\sqrt{2}$

and their positions in the $I$ axis are determined by the function $h(\bar{\rho})$, which satisfies $0 \leq h(\bar{\rho})<\frac{1}{\sqrt{3}}$. The stability of the fixed points $\left\{P_{1}^{ \pm}, Q_{1}^{ \pm}, P_{2}^{ \pm}, Q_{2}^{ \pm}\right\}$can be analysed by evaluating the Greene's residue (2.39):

$$
\begin{aligned}
& R\left(P_{1}^{ \pm}\right)=-R\left(Q_{1}^{ \pm}\right)=\mp \Lambda(a, b, \hat{\rho}, \bar{\rho}) \\
& R\left(P_{2}^{ \pm}\right)=-R\left(Q_{2}^{ \pm}\right)= \pm h(\bar{\rho}) \Lambda(a, b, \hat{\rho}, \bar{\rho})
\end{aligned}
$$

where the function $\Lambda(a, b, \hat{\rho}, \bar{\rho})$, which corresponds to the residue of $P_{1}^{-}$, is given by:

$$
\Lambda=\pi a b J_{0}(\hat{\rho})\left(1+\bar{\rho}^{2}\right)
$$

Given the values of the parameters $a, b, \hat{\rho}$, and $\bar{\rho}$, we can determine the residue of $P_{1}^{-}$, and then, using equations (2.64)-(2.65), compute the residues of the other fixed points $\left\{P_{1}^{+}, Q_{1}^{ \pm}, P_{2}^{ \pm}, Q_{2}^{ \pm}\right\}$. As shown in Fig. 2.15, depending on the value of $\Lambda$, there are five possible stability configurations. The number of elliptic fixed points reduces when $\Lambda$ increases, and the first points to lose stability are the outer ones. In the configuration $\mathrm{V}$ (figure 2.15e), which occurs for $|\Lambda|>\frac{1}{h(\bar{\rho})}$, all the fixed points are hyperbolic. A final remark is regarding the strong stability of the elliptic points in configurations III and IV when $h(\bar{\rho})$ is near to zero $(\bar{\rho}$ greater than and close to $\sqrt{2})$. A high $\Lambda$ can be attained by high values of the parameter $a$ or the effective perturbation $b_{e f}=b J_{0}(\hat{\rho})$, but the islands 
associated to the elliptic points can still survive once the limit $\frac{1}{h(\bar{\rho})}$ goes to infinity when $\bar{\rho} \rightarrow \sqrt{2}^{+}(\bar{\rho}$ greater than and close to $\sqrt{2})$.

An important consequence of the zonal flow bifurcation is the occurrence of nontwist barrier bifurcations. In particular, instead of just one $N T B$, two additional NTBs appear after the zonal flow bifurcation, which occurs when $\bar{\rho}$ increases. The NTB bifurcation is illustrated in Figs. 2.16a and 2.16b: for $\bar{\rho} \lesssim \bar{\rho}_{b}$, only one region with robust spanning KAM curves is present (Fig. 2.16a); increasing $\bar{\rho}$ such that $\bar{\rho}>\bar{\rho}_{b}$ (Fig. 2.16b), two additional NTBs appear. The outer NTBs are separated from the central one by two regions of confined chaotic orbits.

\subsubsection{Robustness of the central shearless curve}

Our interest in studying the robustness of spanning KAM curves as function of $b$ resides on the fact that this parameter is proportional to the amplitude of the drift waves. For a very small Larmor radius (or in the absence of FLR corrections), high amplitude values can easily destroy all spanning KAM curves, what is not always the case when FLR effects are taken into account. As mentioned in section 2.3.3, the robustness of the GSNM's shearless curve can be significantly increased if $\hat{\rho}$ is near a zero of $J_{0}$. In this case, the effective perturbation $b_{e f}$ is small, what explains the robustness of the shearless curve even for high values of $b$. The same effect is present in the GQNM, as we can see in equation (2.57).

Figures 2.17(a)-(d) show breakup diagrams for the central shearless KAM curve as function of $b$ and $\bar{\rho}$. In all cases, $b_{e f}$ is small, because the values of $\hat{\rho}$ are close to the zeros of $J_{0}$. Under this condition, and when $\bar{\rho}$ is near the zonal flow bifurcation threshold $\bar{\rho}_{b}$, defined in (2.56), a significant increasing of the robustness of the shearless curve is observed. For $\bar{\rho}<\bar{\rho}_{b}$, higher values of $b$ are required to the breakup as $\bar{\rho}$ approaches $\bar{\rho}_{b}$. However, for $\bar{\rho}>\bar{\rho}_{b}$, the robustness diminishes as $\bar{\rho}$ increases. Therefore, the central NTB robustness can be increased not only by approximating $\hat{\rho}$ to any of the $J_{0}$ 's zeros, but also by making $\bar{\rho}$ close to $\bar{\rho}_{b}$.

It is interesting to note that, in the neighbourhood of $I=0$, the degree of "flatness" of the frequency's profile has a similar behavior. Like the robustness of the central shearless 


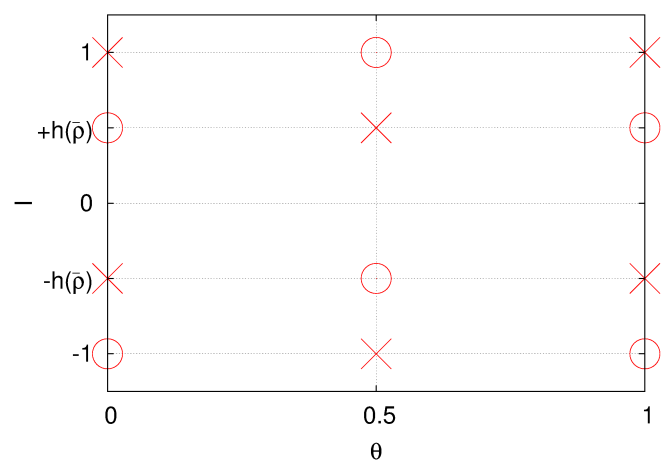

(a) $I: 0<\Lambda<1$

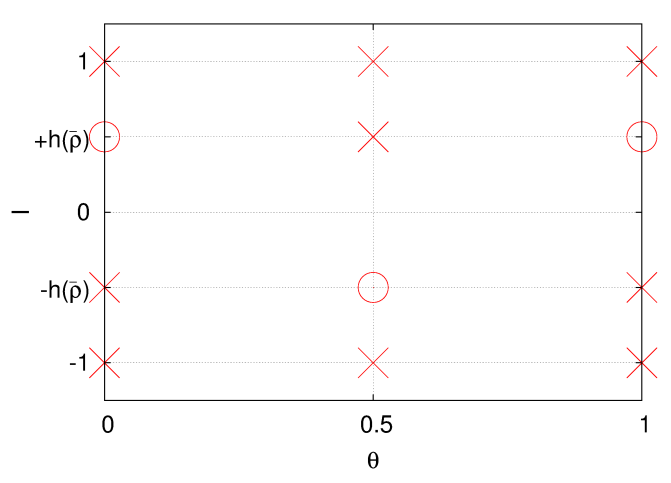

(c) $I I I: 1<\Lambda<\frac{1}{h(\bar{\rho})}$

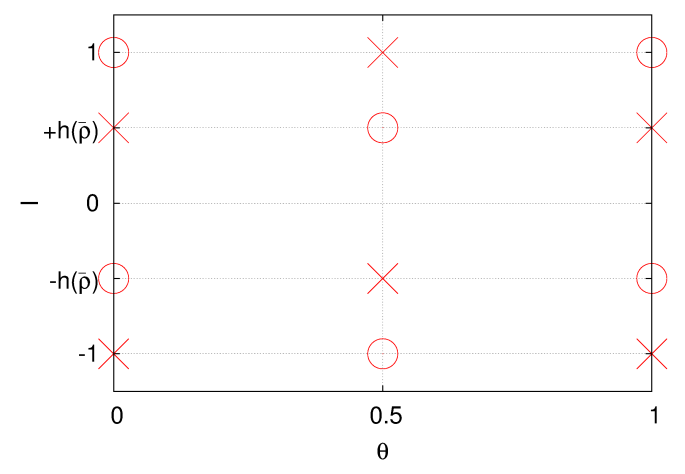

(b) $I I:-1<\Lambda<0$

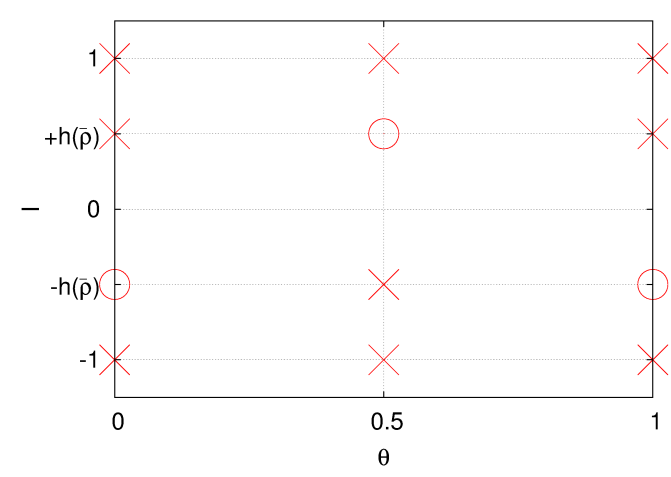

(d) $I V:-\frac{1}{h(\bar{\rho})}<\Lambda<-1$

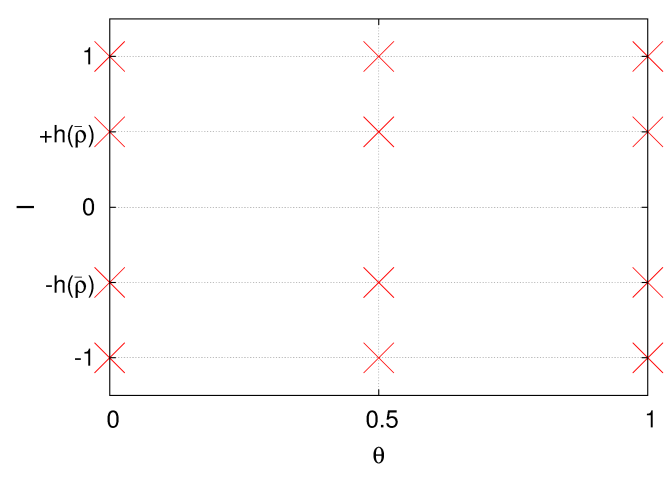

(e) $V: \Lambda<-\frac{1}{h(\bar{\rho})}$ or $+\frac{1}{h(\bar{\rho})}<\Lambda$

Figure 2.15: Depending on the value of $\Lambda$, there are five possible configurations characterizing the stabillity of the period-one fixed points $\left\{P_{1}^{ \pm}, Q_{1}^{ \pm}, P_{2}^{ \pm}, Q_{2}^{ \pm}\right\}$in the GQNM. 


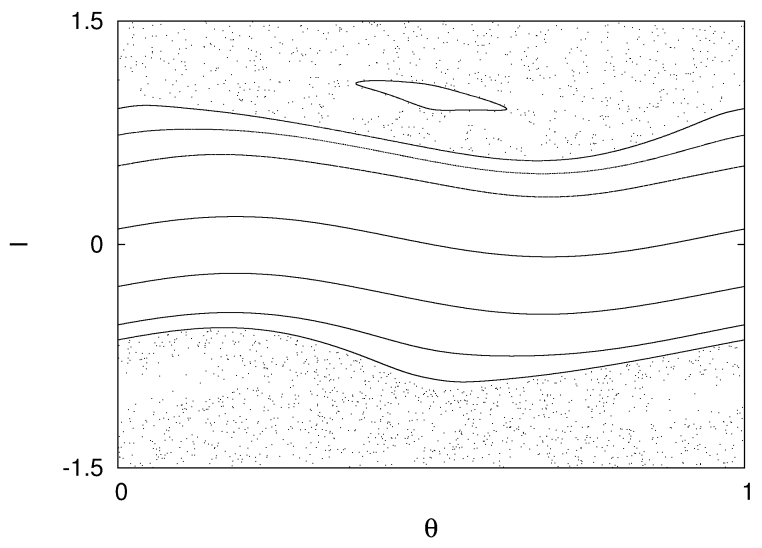

(a) $\bar{\rho}=0.707$

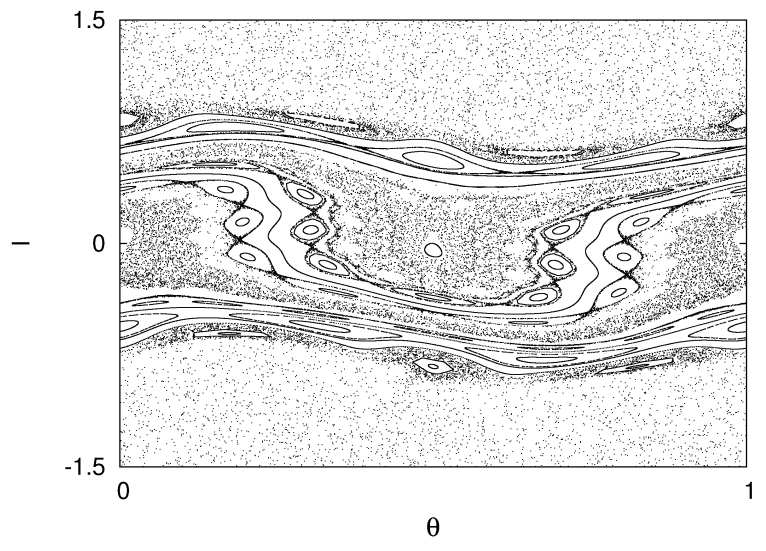

(b) $\bar{\rho}=1.42$

Figure 2.16: (a) One NTB for $\bar{\rho} \lesssim \bar{\rho}_{b}$. (b) After the zonal flow bifurcation, three NTBs can be observed. Parameters: $a=0.5 ; b=1.35 ; \hat{\rho}=2.8$.

curve, the degree of "flatness" is higher near the bifurcation threshold, but it becomes smaller otherwise. The flatness around $I=0$ can be measured through $\left|\Omega^{\prime \prime}(0)\right|$, which corresponds to the absolute value of the second-order derivative of $\Omega$ around $I=0$. A high degree of flatness around $I=0$ means a small value of $\left|\Omega^{\prime \prime}(0)\right|$. Based on these results, we conjecture that the robustness of NTBs in nontwist symplectic maps depends on the flatness of the frequency profile around the points of maximum or minimum (critical points), and that, for small perturbation, the robustness increases with the degree of flatness.

In section 2.3.3, we mentioned that the robustness of a NTB is related to the phenomena referred to as strong KAM stabillity [23]. The strong KAM stabillity argument is based on the idea of overlapping of resonances, as presented by [32], to explain the destruction of invariant tori (spanning KAM curves). Tori located between neighbouring resonances break up when the resonances overlap. Thus, one expects that tori are more resilient to perturbation when resonance widths are smaller. As shown in [23], in 3/2 degrees of freedom nontwist Hamiltonian systems, the width, $\delta \Omega$, of second-order degenerate resonances scales as:

$$
\delta \Omega \sim\left[\epsilon\left|\Omega^{\prime \prime}\left(I_{0}\right)\right|\right]^{2 / 3}
$$

where $\epsilon$ is the small perturbation parameter, and $I_{0}$ the value of the action variable for 


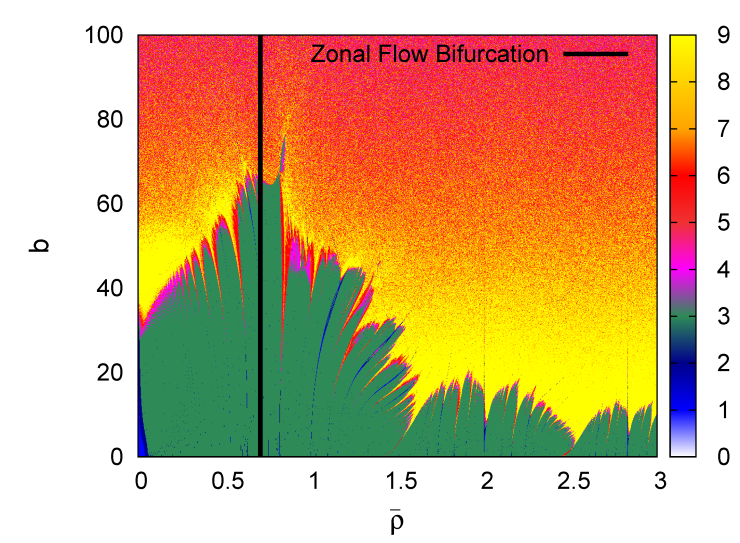

(a) $a=0.5 ; \hat{\rho}=5.47 ; \rho_{2}=5.52$

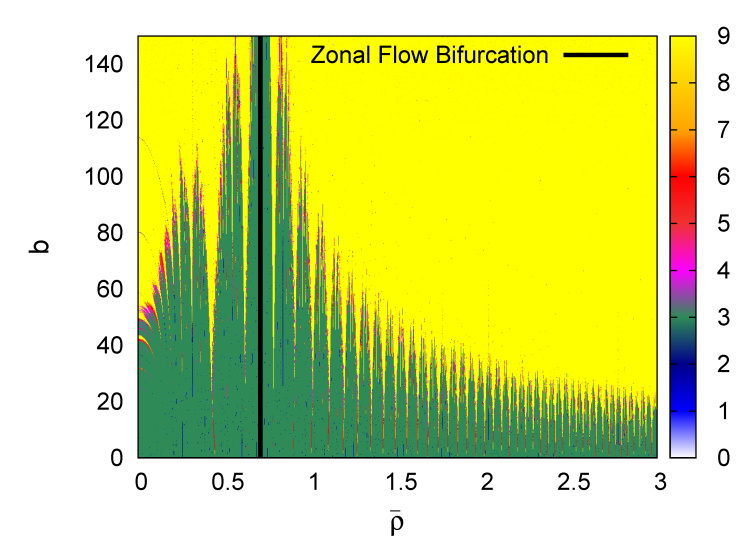

(c) $a=10 ; \hat{\rho}=11.80 ; \rho_{4}=11.79$

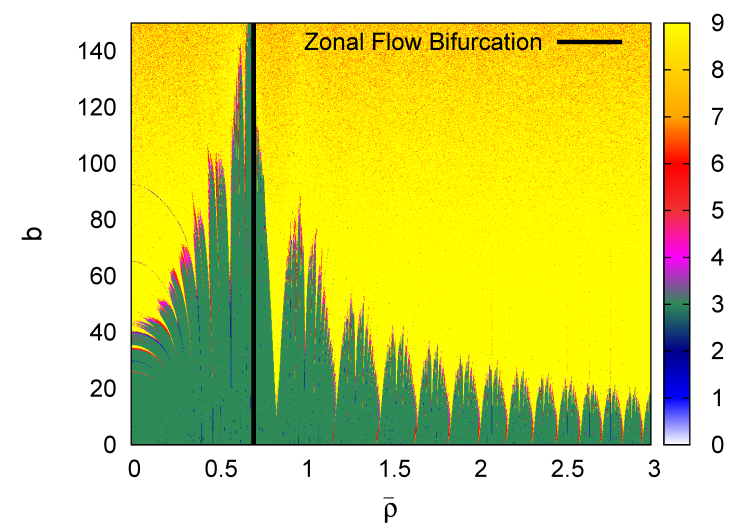

(b) $a=3 ; \hat{\rho}=8.67 ; \rho_{3}=8.65$

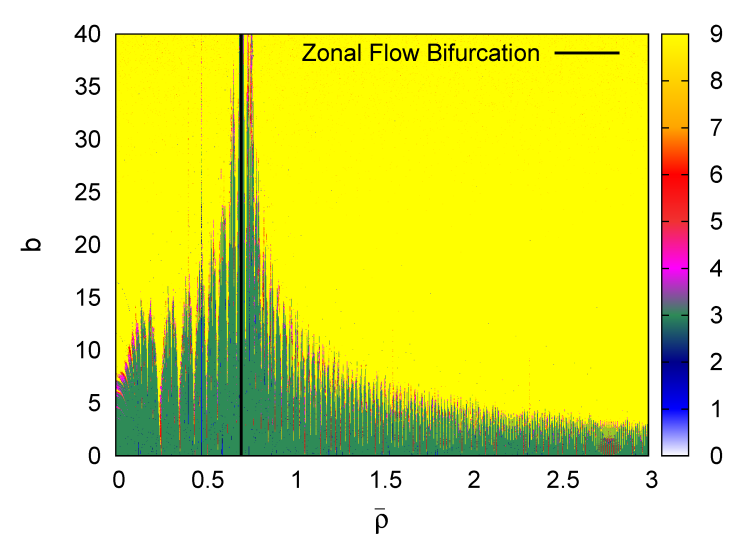

(d) $a=30 ; \hat{\rho}=2.42 ; \rho_{1}=2.40$

Figure 2.17: For small $b_{e f}$ values, the robustness of the central shearless curve (and, thus, also of the central NTB) increases with the degree of flatness of the frequency's profile around the critical point. The flatness can be controlled by the parameter $\bar{\rho}$ and becomes higher when $\bar{\rho}$ is close to the zonal flow bifurcation threshold $\bar{\rho}_{b}$ (shown with the solid black vertical line). The values of $b_{e f}$ are made small by setting $\hat{\rho}$ close to the zeros of $J_{0}$. The first four positive zeros are approximately given by: $\rho_{1}=2.40 ; \rho_{2}=5.52 ; \rho_{3}=8.65 ; \rho_{4}=11.79$. 
which:

$$
\Omega^{\prime}\left(I_{0}\right)=0, \quad \Omega^{\prime \prime}\left(I_{0}\right) \neq 0
$$

As we have seen in section 2.3.3 (see eq. (2.45)), $I_{0}$ in (2.68) corresponds to the value of the action variable for which the frequency's profile violates the twist condition. For $\epsilon=0$, the shearless KAM curve is located exactly at $I_{0}$. If the system is slightly perturbed, secondorder degenerate resonances appear along the neighbourhood of the shearless curve. When these resonances overlap, the shearless curve breaks up.

The central shearless curve of the GQNM is associated to the critical point located at $I_{0}=0$. At this point, the frequency's profile, defined by (2.53), satisfies (2.68) for any $\bar{\rho} \neq \bar{\rho}_{b}$. Thus, in the Hamiltonian system from which the GQNM equations are derived, second-order degenerate resonances might appear near the central shearless curve. According to (2.67) and (2.55), the width of second-order resonances has the property:

$$
\lim _{\bar{\rho} \rightarrow \bar{\rho}_{b}} \delta \Omega=0
$$

because, for $\bar{\rho}$ close to $\bar{\rho}_{b},\left|\Omega^{\prime \prime}\left(I_{0}\right)\right|$ vanishes. Small values of $\left|\Omega^{\prime \prime}\left(I_{0}\right)\right|$ result in small degenerate resonance widths and a more robust central shearless curve. As $\left|\Omega^{\prime \prime}\left(I_{0}\right)\right|$ is directly related to the flatness of the frequency's profile around $I_{0}$ (a small $\left|\Omega^{\prime \prime}\left(I_{0}\right)\right|$ means more "flatness" around $I_{0}$ ), formula (2.67) is consistent with the results showed in figures 2.17(a)-(b) and supports our conjecture relating high robustness of the shearless curve to non-monotonic frequency profiles with high flatness around degenerate points.

It is important to remark that the same arguments developed in this section can be applied to interpret the results presented in Ref. [36], in which the authors study the effect of shearless safety factor profiles on the confinement properties of magnetic field lines in Tokamaks. In the $1+1 / 2$ degrees of fredom Hamiltonian model adopted to describe magnetic field lines, the safety factor $q$ is characterized by regions with vanishing shear that makes the system of a nontwist kind because the $q$-profile is inversely proportional to the nonlinear frequency of system. In Ref. [36], the authors analyze the influence of the amount of flatness in low shear regions of the $q$-profile on the robustness of the NTBs. Changing the form of the $q$-profile, the flatness can be increased, and a more robust NTB can be observed. 


\section{Chapter 3}

\section{Statistical Properties of the GSM Model}

In this chapter we study statistical properties of a GSM based model where the effective perturbation is considered a random variable. Section 3.1 describes the basic assumptions and equations of the model. In section 3.2, the first set of results related to statistics of the effective perturbation are presented: we obtain the gamma's pdf, analyze its main properties and also histograms of gamma for different values of the thermal Lamor radius. The moments, as well the average and dispersion of gamma, are presented in section 3.3. Section 3.4 describes the analytical and numerical results related to the cumulative distributive function of gamma, which is used in section 3.5 to obtain the probability of global chaos. We study the escape rate in section 3.6, and the rate of trapping by period-one islands in section 3.7. This chapter is partly based on Ref. [42].

\subsection{GSM Model}

In the GSM model, particles are assumed to follow orbits described by gyro-averaged standard maps with effective perturbation values which are not necessarily the same. These values differ randomly from one to other particle and are constant in time. The model comes from the idea of considering an ensemble of particles whose Larmor radii are randomly defined and kept constant in time. 
Therefore, the dynamics of each charged particle in the GSM model is determined by:

$$
I_{n+1}=I_{n}+K(\hat{\rho}) \sin \theta_{n}, \quad \theta_{n+1}=\theta_{n}+I_{n+1}, \quad \bmod 2 \pi
$$

where $K$, the effective perturbation, is a random variable defined by:

$$
K=K_{0} J_{0}(\hat{\rho})
$$

and $K_{0}$ denotes the perturbation parameter, which is a constant and the same for all particles of the ensemble ${ }^{1}$.

In a similar a way as done in $[6,9]$, we assume plasmas in thermal equilibrium and a Larmor radius' pdf resulting from the Maxwell-Boltzmann distribution. The Larmor radius' pdf characterizes the statistics of the random variable $\hat{\rho}$ and can be obtained as follows.

\subsubsection{Maxwell-Boltzmann Distribution}

The Maxwell-Boltzmann distribution is given by [43]:

$$
F(\vec{v})=n\left(\frac{\beta}{2 \pi}\right)^{\frac{3}{2}} \exp \left(-\frac{\beta}{2} \vec{v}^{2}\right),
$$

where $\vec{v}$ is the three-dimensional particles' velocity and $n$ is the number of particles per unit volume. The parameter $\beta$ is defined by:

$$
\beta=\frac{k_{B} T}{m},
$$

where $k_{B}$ is the Boltzmann constant, $T$ is the absolute temperature and $m$ defines the particle's mass. Equation (3.3) represents the most probable distribution function satisfying the macroscopic conditions or constraints imposed on the system [43].

According to (3.3), the probability density function of the particle's velocity in one dimension is a Gaussian with zero mean (see [43] for details):

$$
f_{i}\left(v_{i}\right)=\sqrt{\frac{\beta}{2 \pi}} \exp \left(-\frac{\beta}{2} v_{i}^{2}\right),
$$

\footnotetext{
${ }^{1}$ Although the set of Eqs. (3.1) corresponds exactly to th GSM, here we decided to change notation used in the previous chapter to refer to the effective perturbation and the perturbation parameter. $K_{e f}$ is now simply $K$, and the perturbation parameter, previously denoted by $K$, is now $K_{0}$.
} 
where the index $i$ denotes the $x, y$, and $z$ components of the velocity. The thermal velocity is defined as [44]:

$$
v_{t h}=\frac{1}{\sqrt{\beta}}
$$

The thermal velocity $v_{t h}$ corresponds to the standard deviation of the particle's velocity in one dimension:

$$
v_{t h}=\sigma_{v_{i}}=\sqrt{\left\langle v_{i}^{2}\right\rangle-\left\langle v_{i}\right\rangle^{2}}
$$

Equation (3.7) can be verified considering that $\left\langle v_{i}\right\rangle=0$ and computing the moment $\left\langle v_{i}^{2}\right\rangle$ :

$$
\left\langle v_{i}^{2}\right\rangle=\int_{-\infty}^{+\infty} f_{i}\left(v_{i}\right) v_{i}^{2} d v_{i}=\frac{1}{\beta} .
$$

Using (3.7), equation (3.5) can rewritten as:

$$
f_{i}\left(v_{i}\right)=\frac{1}{\sqrt{2 \pi v_{t h}^{2}}} \exp \left(-\frac{v_{i}^{2}}{2 v_{t h}^{2}}\right) .
$$

\subsubsection{Larmor Radius' PDF}

The Larmor radius is defined by [44]:

$$
\rho=\frac{v_{\perp}}{\Omega_{0}}
$$

where the cyclotron frequency $\Omega_{0}$ is:

$$
\Omega_{0}=\frac{|q| B_{0}}{m}
$$

and $v_{\perp}$ corresponds to the perpendicular component of the particle's velocity to the toroidal magnetic field lines:

$$
v_{\perp}=\sqrt{v_{x}^{2}+v_{y}^{2}}
$$

The probability of the perpendicular velocity assuming a value between $v_{\perp}$ and $v_{\perp}+$ $d v_{\perp}$, denoted by $f_{\perp}\left(v_{\perp}\right) d v_{\perp}$, corresponds to the probability of having $\vec{v}_{\perp}=\left(v_{x}, v_{y}\right)$ inside 
a circular region $R$ of radii $v_{\perp}$ and $v_{\perp}+d v_{\perp}$, i.e.:

$$
\begin{aligned}
f_{\perp}\left(v_{\perp}\right) d v_{\perp} & =\int_{R} f_{x}\left(v_{x}\right) f_{y}\left(v_{y}\right) d v_{x} d v_{y} \\
& =\frac{\beta}{2 \pi} \int_{R} \exp \left[-\frac{\beta}{2}\left(v_{x}^{2}+v_{y}^{2}\right)\right] d v_{x} d v_{y} \\
& =\frac{\beta}{2 \pi} \int_{0}^{2 \pi} \exp \left(-\frac{\beta}{2} v_{\perp}^{2}\right) v_{\perp} d \theta d v_{\perp} .
\end{aligned}
$$

Thus, the perpendicular velocity's pdf is:

$$
f_{\perp}\left(v_{\perp}\right)=\beta v_{\perp} \exp \left(-\frac{\beta}{2} v_{\perp}^{2}\right)
$$

The probability of finding the Larmor radius between $\rho$ and $\rho+d \rho$ is $f_{L}(\rho) d \rho$, where $f_{L}$ is the Larmor radius' pdf. According to Eq. (3.10), if we assume that the cyclotron frequency is a constant and the same for all charged particles, then:

$$
\begin{aligned}
f_{L}(\rho) d \rho & =f_{\perp}\left(v_{\perp}\right) d v_{\perp} \\
& =\beta \Omega_{0}^{2} \rho \exp \left(-\frac{\beta \Omega_{0}^{2}}{2} \rho^{2}\right) d \rho
\end{aligned}
$$

and:

$$
f_{L}(\rho)=\beta \Omega_{0}^{2} \rho \exp \left(-\frac{\beta \Omega_{0}^{2}}{2} \rho^{2}\right)
$$

Defining the thermal Lamor radius as:

$$
\rho_{t h}=\frac{v_{t h}}{\Omega_{0}}=\frac{1}{\Omega_{0} \sqrt{\beta}}
$$

and substituting (3.20) in (3.19), we have:

$$
f_{L}(\rho)=\frac{\rho}{\rho_{t h}^{2}} \exp \left[-\frac{1}{2}\left(\frac{\rho}{\rho_{t h}}\right)^{2}\right]
$$

In chapter 2, we defined the normalized Lamor radius as $\hat{\rho}=k \rho$. According to (3.21), the normalized Lamor radius' pdf is then given by:

$$
f(\hat{\rho})=\frac{\hat{\rho}}{\hat{\rho}_{t h}^{2}} \exp \left[-\frac{1}{2}\left(\frac{\hat{\rho}}{\hat{\rho}_{t h}}\right)^{2}\right],
$$




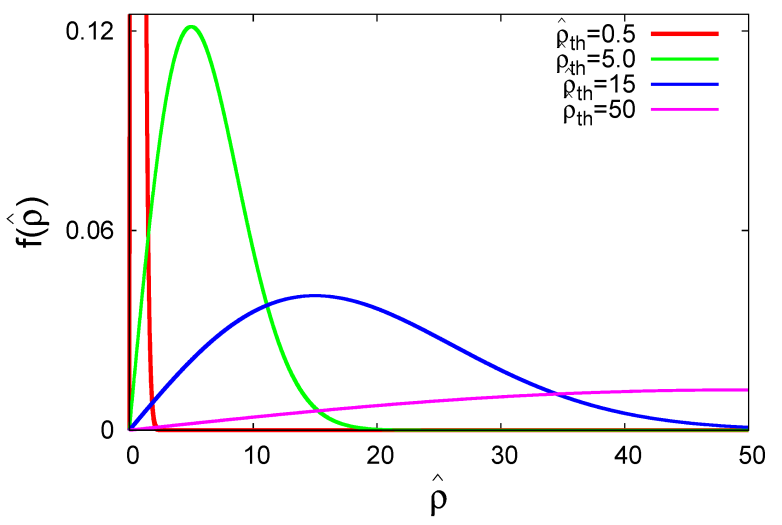

Figure 3.1: Probability density function of $\hat{\rho}$, Eq. (3.22), for different values of the thermal Larmor radius, $\hat{\rho}_{t h}$.

where $\hat{\rho}_{t h}$ denotes the normalized thermal Larmor radius, which we define as $\hat{\rho}_{t h}=k \rho_{t h}$. Using (3.4), (3.11) and (3.20), the parameter $\hat{\rho}_{t h}$ can also be written as:

$$
\hat{\rho}_{t h}=\frac{k}{|q| B_{0}} \sqrt{m k_{B} T} .
$$

Together with (3.1), the pdf (3.22) completes the set of equations of the GSM model. For the sake of brevity will call from here on the dimensionless variable $\hat{\rho}$ simply "Larmor radius". Thus, we will refer to $\hat{\rho}_{t h}$ and $f(\hat{\rho})$ simply as "thermal Larmor radius" and "Larmor radius' pdf", respectively.

Figure 3.1 shows plots of (3.22) for different values of the thermal Larmor radius. The PDF has a maximum at $\hat{\rho}_{t h}$, which means that $\hat{\rho}_{t h}$ is the most probable Larmor radius. The average and variance associated to $\hat{\rho}$ can be easily obtained from (3.22) and are both dependent on $\hat{\rho}_{t h}$. The average $\langle\hat{\rho}\rangle$ is given by:

$$
\langle\hat{\rho}\rangle=\sqrt{\frac{\pi}{2}} \hat{\rho}_{t h},
$$

and the variance, defined by $\sigma^{2}=\left\langle\hat{\rho}^{2}\right\rangle-\langle\hat{\rho}\rangle^{2}$, is:

$$
\sigma^{2}=\frac{4-\pi}{2} \hat{\rho}_{t h}^{2}
$$

\subsection{Gamma's Probability Density Function}

Let $\gamma$ be defined as ratio between the effective perturbation $K$ and the perturbation parameter $K_{0}$. According to Eq. (3.2), $\gamma$ is a random variable depending on the particle's 
Larmor radius and is given by $\gamma=J_{0}(\hat{\rho})$

Applying the random variable transformation theorem [45], the probability density function (PDF) of $\gamma$ can be written as:

$$
g(\gamma)=\int_{0}^{\infty} \delta\left[\gamma-J_{0}(\hat{\rho})\right] f(\hat{\rho}) d \hat{\rho}
$$

For a given value of $\gamma$, let $\Gamma_{\gamma}=\left\{\hat{\rho}_{0}, \hat{\rho}_{1}, \hat{\rho}_{2}, \ldots\right\}$ be the set of non-negative solutions $\hat{\rho}_{i}$ in equation $\gamma=J_{0}\left(\hat{\rho}_{i}\right)$ such that $J_{0}^{\prime}\left(\hat{\rho}_{i}\right) \neq 0$. If $\Gamma_{\gamma}$ is a non-empty set, the Dirac delta function in (3.26) can rewritten as [46]:

$$
\delta\left[\gamma-J_{0}(\hat{\rho})\right]=\sum_{\hat{\rho}_{i} \in \Gamma_{\gamma}} \frac{\delta\left(\hat{\rho}-\hat{\rho}_{i}\right)}{\left|J_{0}^{\prime}\left(\hat{\rho}_{i}\right)\right|}
$$

where $J_{0}^{\prime}$ is the derivative of $J_{0}$. We note that $J_{0}^{\prime}(\hat{\rho})=-J_{1}(\hat{\rho})$, where $J_{1}$ is the first-order bessel function.

Substituting (3.27) in (3.26), we have:

$$
g(\gamma)=\frac{1}{\hat{\rho}_{t h}^{2}} \sum_{\hat{\rho}_{i} \in \Gamma_{\gamma}} \frac{\hat{\rho}_{i}}{\left|J_{0}^{\prime}\left(\hat{\rho}_{i}\right)\right|} \exp \left[-\frac{1}{2}\left(\frac{\hat{\rho}_{i}}{\hat{\rho}_{t h}}\right)^{2}\right], \quad \gamma_{\min }<\gamma<\gamma_{\max }
$$

where the interval limits $\gamma_{\min }$ and $\gamma_{\max }$ are the smallest minimum and the greatest maximum of $J_{0}: \gamma_{\min } \simeq-0.4$ and $\gamma_{\max }=1$. Outside the interval $\gamma_{\min }<\gamma<\gamma_{\max }, \Gamma_{\gamma}$ is an empty set and $g(\gamma)=0$.

We note that a PDF equivalent to (3.28) was also obtained in Ref. [9], where the same mathematical problem arises, although the models and related problems considerer there are different from those studied in this work.

Function $g$ consists of a sum of terms involving the product of two other functions: $f(\hat{\rho})$, defined by $(3.22)$, and $s(\hat{\rho})=1 /\left|J_{0}^{\prime}(\hat{\rho})\right|$, both of them evaluated at values $\hat{\rho}=\hat{\rho}_{i}$ such that $\gamma=J_{0}\left(\hat{\rho}_{i}\right)$. If $\gamma$ is near a maximum or a minimum of $J_{0}$, function $s$ diverges. Thus, the minima and maxima of $J_{0}$ correspond to singularities of $g$.

The singularities' locations are indicated by the blue vertical lines of Fig. 3.2. The singularities occur in large number near $\gamma=0$ at positive and negative positions. This property can be explained by the asymtotic behavior of zero-order Bessel function for high arguments. Since $J_{0}$ is an oscillatory and decaying function varying like $J_{0}(\hat{\rho}) \sim$ 


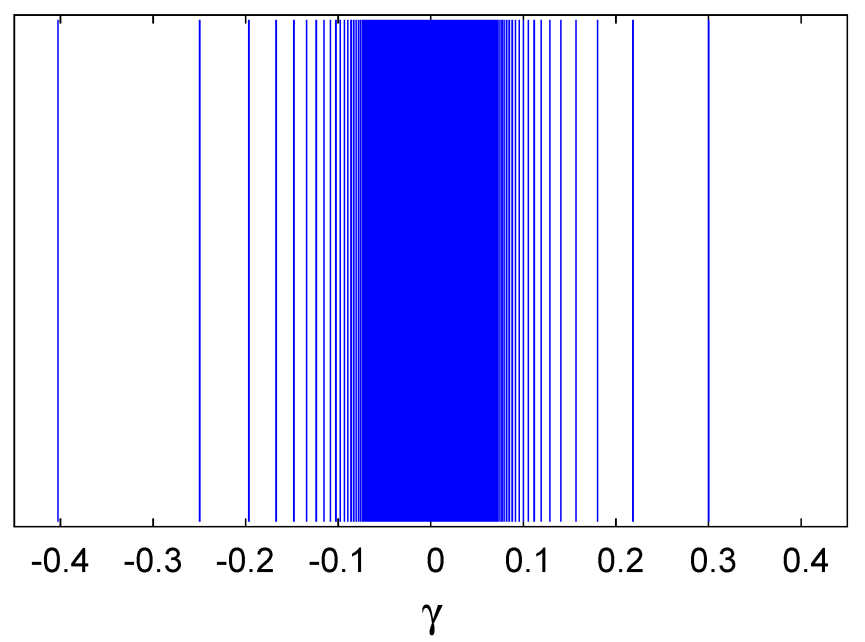

Figure 3.2: Vertical lines: location of singularities

$\sqrt{\frac{2}{\pi \hat{\rho}}} \cos \left(\hat{\rho}-\frac{\pi}{4}\right)$ for high values of $\hat{\rho}[47], J_{0}$ has an infinite number of maxima and minima near zero. Thus, an infinite number of singularities concentrate near $\gamma=0$.

Figure 3.3 shows the vertical lines that indicate the singularities superimposed to histograms of $\gamma$. To construct each histogram, we proceeded as following. A set of random Larmor radii is numerically generated according to the PDF defined by Eq. (3.22). For each random Larmor radius $\hat{\rho}$ generated, the corresponding value of $\gamma$ is computed using the relation $\gamma=J_{0}(\hat{\rho})$. The histograms are normalized by the total number of $\gamma$ values generated.

The main feature exhibited by the histograms of Fig. 3.3 is the occurence of "peaks" near the singularities. Although peaks do not emerge in all singularities, if a certain peak exists, it occurs near one of the singularities. Here we call the singularity as "strong" in case that an associated "peak" exists or "weak" when no peak is observed in the histogram.

The fast exponential decaying character of function $f$, present in all terms of $g$, controls the effect of each singularity, acting as an "height" function and limiting the growth of $g(\gamma)$. In Fig. 3.4(a), the histogram shown in Fig. 3.3(c) is compared to function $f$ (points), which is evaluated at values $\hat{\rho}^{*}$ for which $J_{0}\left(\hat{\rho}^{*}\right)$ defines the position of a singularity. As can be seen in Fig. 3.4(a), peaks occur where $f$ has higher values and the singularities are more distant to each other. The peaks can not be observed near $\gamma=0$ where $f$ goes 


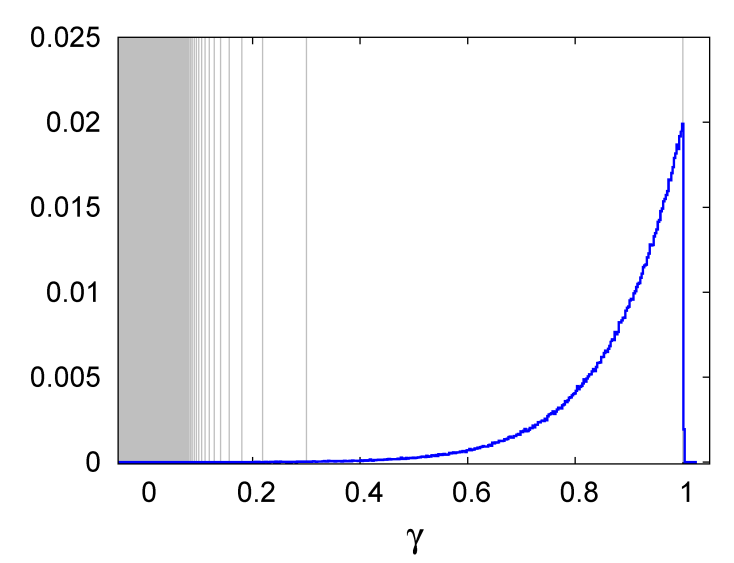

(a) $\hat{\rho}_{t h}=0.5$

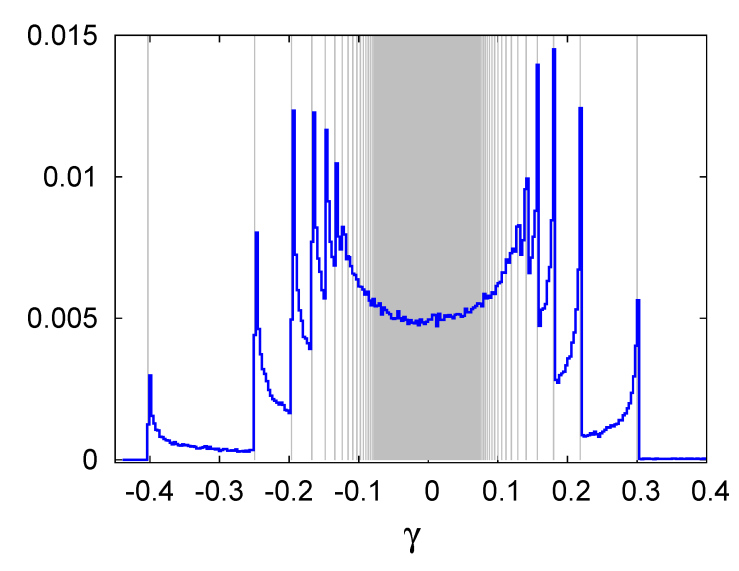

(c) $\hat{\rho}_{t h}=15$

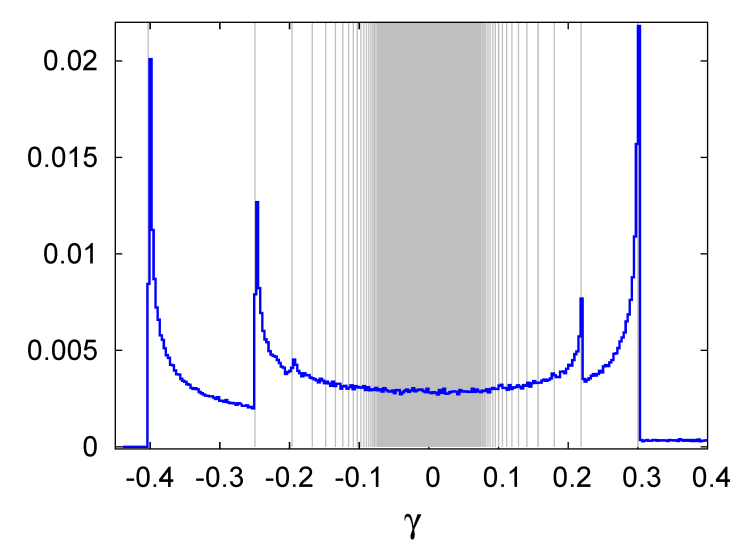

(b) $\hat{\rho}_{t h}=5$

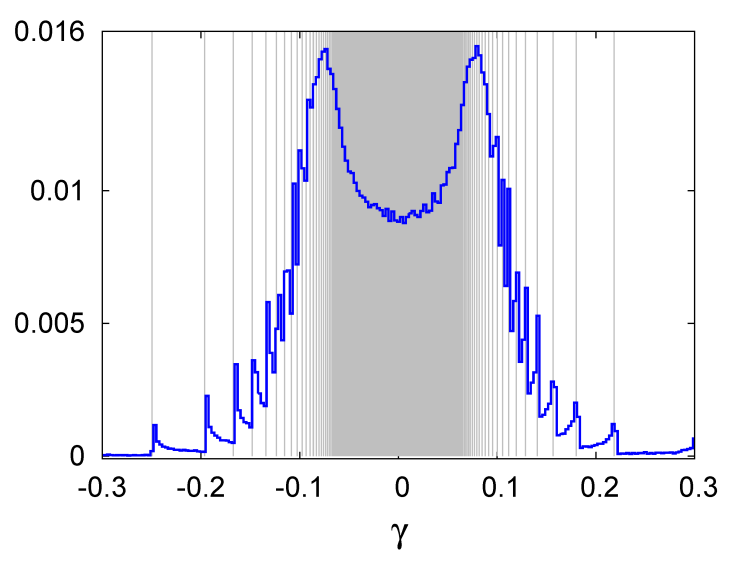

(d) $\hat{\rho}_{t h}=50$

Figure 3.3: Histograms of $\gamma$ superimposed to the vertical lines indicating the singularities. 


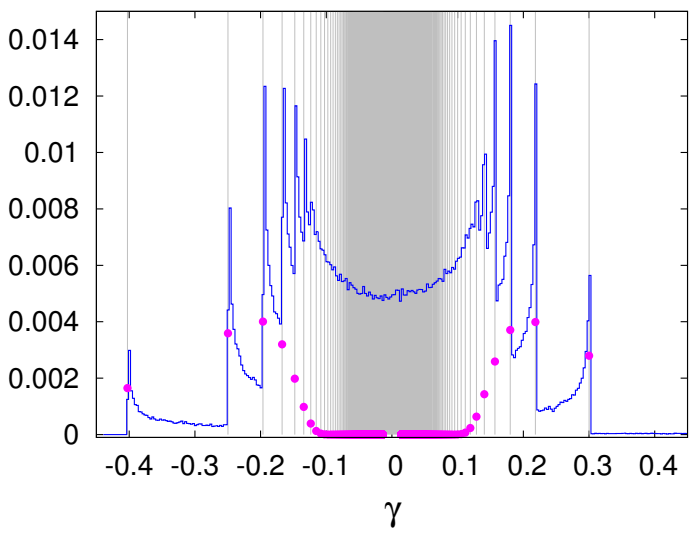

(a) Function $f$ at the singularities and the histogram of Fig. 3.3(c).

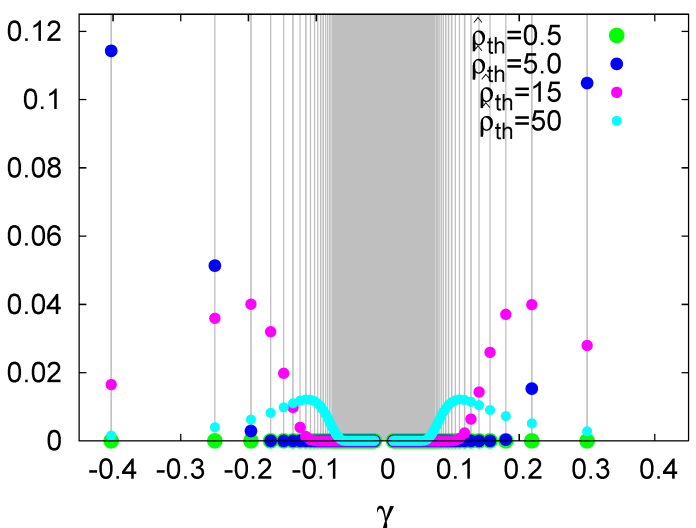

(b) Function $f$ at the singularities for different values of $\hat{\rho}_{t h}$.

Figure 3.4: Function $f$, defined by Eq.(3.22), evaluated at the points of singularity. The fast exponential decaying character of $f$ controls the effects of singularities, acting as an "height" function and limiting the growth of $g(\gamma)$.

to zero and the singularities are more concentrated. Thus, function $f$ determines what singularities can be considered as strong or weak.

Fig. 3.4(b) shows other plots of $f$, also evaluated at the singularity points and using different values of $\hat{\rho}_{t h}$, the same ones used in the histograms of Figs. 3.3(a)-(c). Comparing the histograms with the plots in Fig. 3.4(b), we see again peaks emerging at singularities with the highest values of $f$ and disappearing at singularities where $f$ goes to zero.

\subsection{Gamma's Average and Dispersion}

The $n$-th moment of $\gamma$ is given by:

$$
\left\langle\gamma^{n}\right\rangle=\int_{-\infty}^{+\infty} \gamma^{n} g(\gamma) d \gamma
$$

Substituting (3.26) in (C.27), we have:

$$
\left\langle\gamma^{n}\right\rangle=\frac{1}{\hat{\rho}_{t h}^{2}} \int_{0}^{+\infty}\left[J_{0}(\hat{\rho})\right]^{n} \exp \left[-\frac{1}{2}\left(\frac{\hat{\rho}}{\hat{\rho}_{t h}}\right)^{2}\right] \hat{\rho} d \hat{\rho} .
$$

The first-moment, $\langle\gamma\rangle$, can be computed using (3.30) and Eq. (6.631-4) of Ref. [48] 
(pg. 706):

$$
\int_{0}^{+\infty} \hat{\rho}^{\nu+1} e^{-\alpha \hat{\rho}^{2}} J_{\nu}(\beta \hat{\rho}) d \hat{\rho}=\frac{\beta^{\nu}}{(2 \alpha)^{\nu+1}} \exp \left(-\frac{\beta^{2}}{4 \alpha}\right),
$$

where the parameters $\alpha, \beta$, and $\nu$ are here defined as: $\alpha=\frac{1}{2 \hat{\rho}_{t h}^{2}}, \beta=1$, and $\nu=0$. Setting $n=1$ and substituting (3.31) in (3.30), we obtain:

$$
\langle\gamma\rangle=\exp \left(-\frac{\hat{\rho}_{t h}^{2}}{2}\right)
$$

Thus, the average of $\gamma$ is near one for small values of the thermal Larmor radius and decreases exponentially to zero for increasing values of that radius. Since $\gamma=K / K_{0}$, the average of the effective perturbation, according to (3.32), is given by:

$$
\langle K\rangle=K_{0} \exp \left(-\frac{\hat{\rho}_{t h}^{2}}{2}\right)
$$

The second moment, $\left\langle\gamma^{2}\right\rangle$, can be determined using Eq. (6.633-2) of Ref. [48] (pq. 707):

$$
\int_{0}^{+\infty} \hat{\rho} e^{-\varrho^{2} \hat{\rho}^{2}} J_{p}(\alpha \hat{\rho}) J_{p}(\beta \hat{\rho}) d \hat{\rho}=\frac{1}{2 \varrho^{2}} \exp \left[\frac{\alpha^{2}+\beta^{2}}{4 \varrho^{2}}\right] I_{p}\left(\frac{\alpha \beta}{2 \varrho^{2}}\right) .
$$

Let $\varrho^{2}=\frac{1}{2 \hat{\rho}_{t h}^{2}}, \alpha=\beta=1$, and $p=0$. Substituting (3.34) in (3.30), with $n=2$, the second moment is:

$$
\left\langle\gamma^{2}\right\rangle=e^{-\hat{\rho}_{t h}^{2}} I_{0}\left(\hat{\rho}_{t h}^{2}\right)
$$

Finally, using (3.32) and (3.35), the dispersion of $\gamma$, defined by $\sigma_{\gamma}^{2}=\left\langle\gamma^{2}\right\rangle-\langle\gamma\rangle^{2}$, can be written as:

$$
\sigma_{\gamma}^{2}=e^{-\hat{\rho}_{t h}^{2}}\left[I_{0}\left(\hat{\rho}_{t h}^{2}\right)-1\right]
$$

The dispersion of the effective perturbation is then given by:

$$
\sigma_{K}^{2}=K_{0}^{2} e^{-\hat{\rho}_{t h}^{2}}\left[I_{0}\left(\hat{\rho}_{t h}^{2}\right)-1\right]
$$

As can be seen in Fig. 3.5, the dispersion increases for small values of $\hat{\rho}_{t h}$ from zero to a maximum and then starts to decay. For large $\hat{\rho}_{t h}$, since $I_{0}\left(\hat{\rho}_{t h}^{2}\right) \sim \frac{e^{+\hat{\rho}_{t h}^{2}}}{\sqrt{2 \pi \hat{\rho}_{t h}^{2}}}[47], \sigma_{\gamma}^{2}$ decays as $\sigma_{\gamma}^{2} \sim \frac{1}{\hat{\rho}_{t h}}$. Thus, the dispersion "expands" from zero to a maximum and then "compress" to zero again for increasing $\hat{\rho}_{t h}$. 


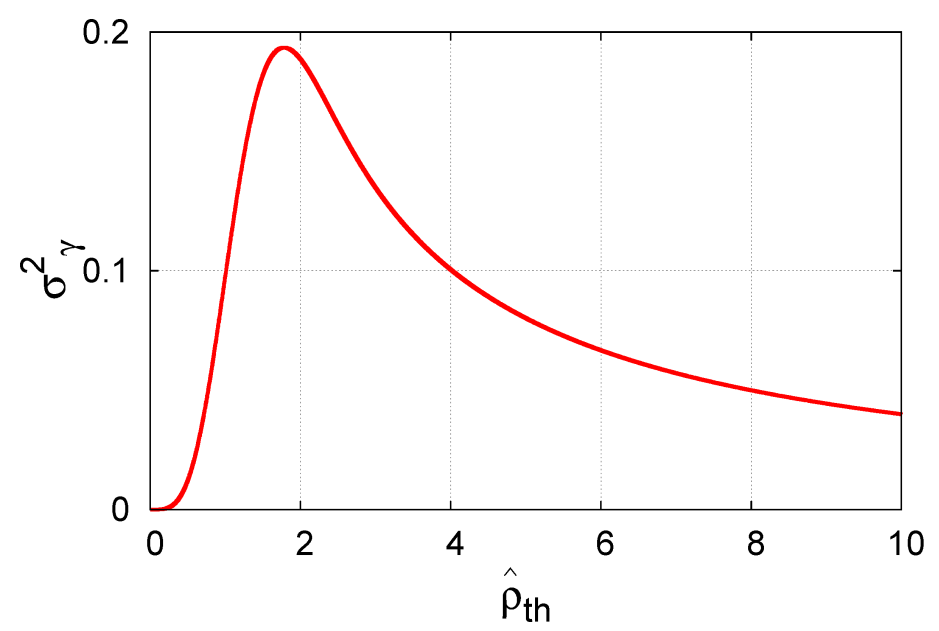

Figure 3.5: Dispersion of $\gamma, \sigma_{\gamma}^{2}$. For increasing $\hat{\rho}_{t h}, \sigma_{\gamma}^{2}$ increases from zero to maximum and then goes to zero again at high values of $\hat{\rho}_{t h}$.

The histograms shown in Figs. 3.3(a)-(d) also indicate that, for increasing $\hat{\rho}_{t h}$, both the average and the dispersion goes to zero because the peaks become more symmetrically concentrated near $\gamma=0$, what is specially evident in the histogram of Fig. 3.3(d).

\subsection{Gamma's Cumulative Distribution Function}

The cumulative distribution function of $\gamma$, denoted by $G(\gamma)$, is defined as:

$$
G(\gamma)=\int_{-\infty}^{\gamma} g\left(\gamma^{\prime}\right) d \gamma^{\prime}
$$

Substituting (3.26) in (3.38), we have:

$$
G(\gamma)=\int_{0}^{\infty} \Theta\left[\gamma-J_{0}(\hat{\rho})\right] f(\hat{\rho}) d \hat{\rho}
$$

where $\Theta$ is the Heaviside step function. A partial integration in (3.39) yields:

$$
G(\gamma)=\Theta(\gamma-1)-\int_{0}^{\infty} \delta\left[\gamma-J_{0}(\hat{\rho})\right] J_{0}^{\prime}(\hat{\rho}) \exp \left[-\frac{1}{2}\left(\frac{\hat{\rho}}{\hat{\rho}_{t h}}\right)^{2}\right] d \hat{\rho}
$$

If $\gamma$ is outside the interval $\gamma_{\min }<\gamma<\gamma_{\max }$, the second term in (3.40) is zero, and:

$$
G(\gamma)=\Theta(\gamma-1)
$$


which means that $G(\gamma)=0$ for $\gamma \leq \gamma_{\min }$ and $G(\gamma)=1$ for $\gamma \geq \gamma_{\max }$.

If $\gamma_{\min }<\gamma<\gamma_{\max }, \Theta(\gamma-1)=0$ and, using again formula (3.27), Eq. (3.40) yields:

$$
G(\gamma)=-\sum_{\hat{\rho}_{i} \in \Gamma_{\gamma}} \frac{J_{0}^{\prime}\left(\hat{\rho}_{i}\right)}{\left|J_{0}^{\prime}\left(\hat{\rho}_{i}\right)\right|} \exp \left[-\frac{1}{2}\left(\frac{\hat{\rho}_{i}}{\hat{\rho}_{t h}}\right)^{2}\right]
$$

A simpler form for (3.42) can be obtained if we define an additional property for $\Gamma_{\gamma}$. Let $\Gamma_{\gamma}$ be an order set such that $\hat{\rho}_{0}<\hat{\rho}_{1}<\hat{\rho}_{2}<\ldots$ Each solution $\hat{\rho}_{i}$ belongs to an interval where $J_{0}(\hat{\rho})$ is increasing or decreasing. $J_{0}(\hat{\rho})$ oscillates such that $J_{0}^{\prime}\left(\hat{\rho}_{i}\right) /\left|J_{0}^{\prime}\left(\hat{\rho}_{i}\right)\right|=-1$ for $i=0,2,4, .$. and $J_{0}^{\prime}\left(\hat{\rho}_{i}\right) /\left|J_{0}^{\prime}\left(\hat{\rho}_{i}\right)\right|=+1$ for $i=1,3,5, .$. , or:

$$
J_{0}^{\prime}\left(\hat{\rho}_{i}\right) /\left|J_{0}^{\prime}\left(\hat{\rho}_{i}\right)\right|=(-1)^{i-1}, \quad i=0,1,2, \ldots
$$

Substituting (3.43) in (3.42), we have the following set of equations for the cumulative distribution function:

$$
G(\gamma)=\left\{\begin{array}{cc}
0, & \gamma \leq \gamma_{\min } \\
\sum_{\hat{\rho}_{i} \in \Gamma_{\gamma}}(-1)^{i} \exp \left[-\frac{1}{2}\left(\frac{\hat{\rho}_{i}}{\hat{\rho}_{t h}}\right)^{2}\right], & \gamma_{\min }<\gamma<\gamma_{\max } \\
1 & \gamma \geq \gamma_{\max }
\end{array}\right.
$$

In Figs. 3.6(a)-(b), using different values of $\hat{\rho}_{t h}$, we show plots of (3.44) (red curves) compared to numerical simulations of $G$ (black points). Figures 3.6(a)-(b) show that both analytical and numerical results are in good agreement. The numerical simulations are performed as follows: first, we generate a set of random Larmor radii; second, for each Larmor radius value $\hat{\rho}$ generated, we compute $J_{0}(\hat{\rho})$; finally, for a given $\gamma$, we determine the rate or frequency of values $J_{0}(\hat{\rho})$ such that $J_{0}(\hat{\rho}) \leq \gamma$. The total number of random values generated is 1000 .

As shown in Figs. 3.6(a)-(b), the main properties of $G(\gamma)$ are the following:

- $G(\gamma)$ goes to zero if $\gamma$ is near $\gamma_{\text {min }}$, since no value of $J_{0}$ can be below $\gamma_{\text {min }}$.

- $G(\gamma)$ goes to one if $\gamma$ is near $\gamma_{\max }$, since no value of $J_{0}$ can be above $\gamma_{\max }$.

- $G(\gamma)$ is not a "smooth" function, what means that its derivative have descontinuities. 


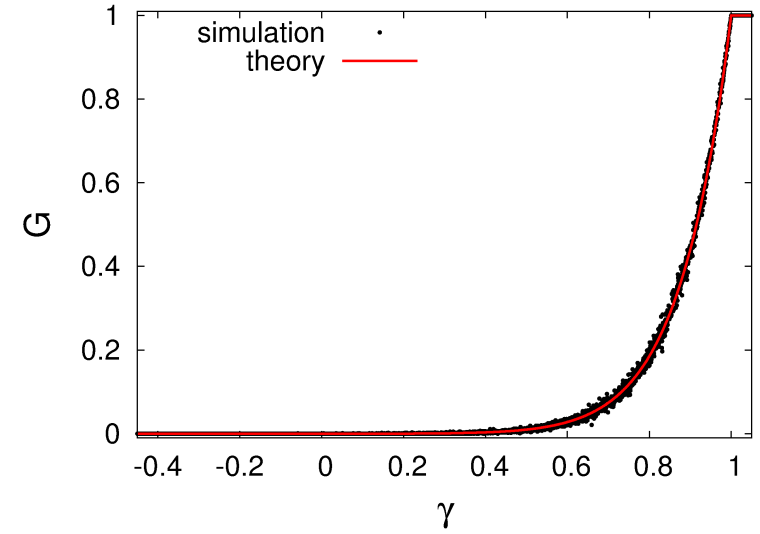

(a) $\hat{\rho}_{t h}=0.5$

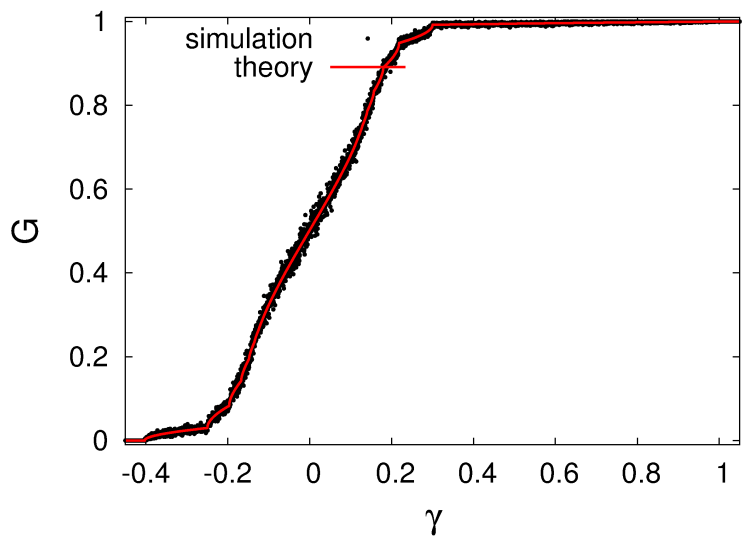

(c) $\hat{\rho}_{t h}=15$

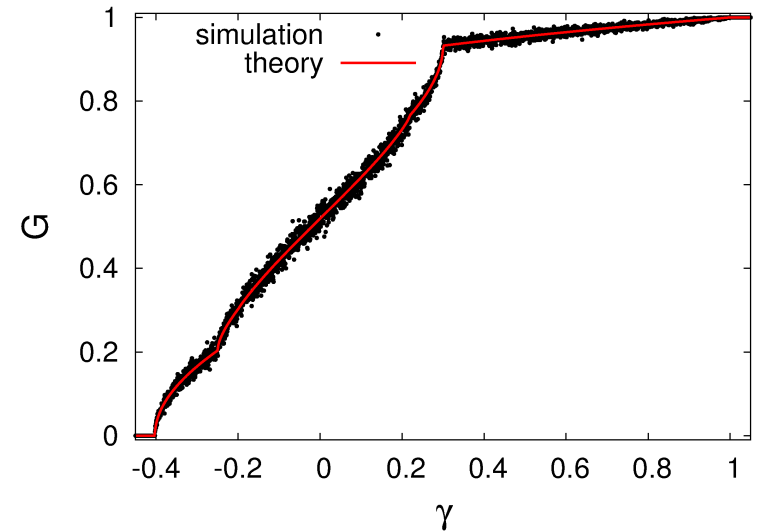

(b) $\hat{\rho}_{t h}=5.0$

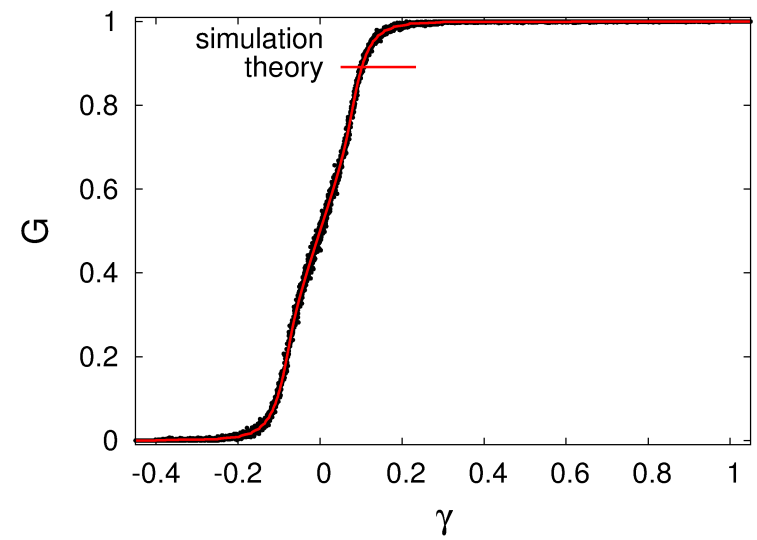

(d) $\hat{\rho}_{t h}=50$

Figure 3.6: Cumulative distribution function of $\gamma$, denoted by $G(\gamma)$ The red curves are plots of the analytical result described by Eq. (3.44). The black points correspond to numerical simulations. 


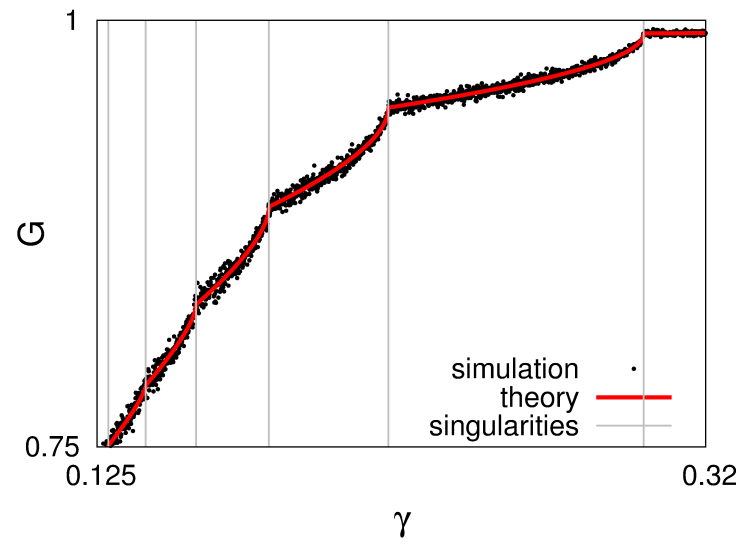

(a)

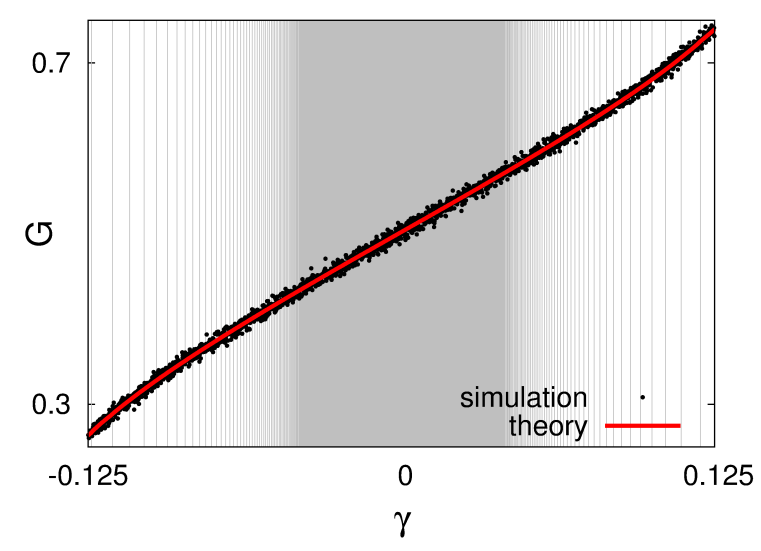

(b)

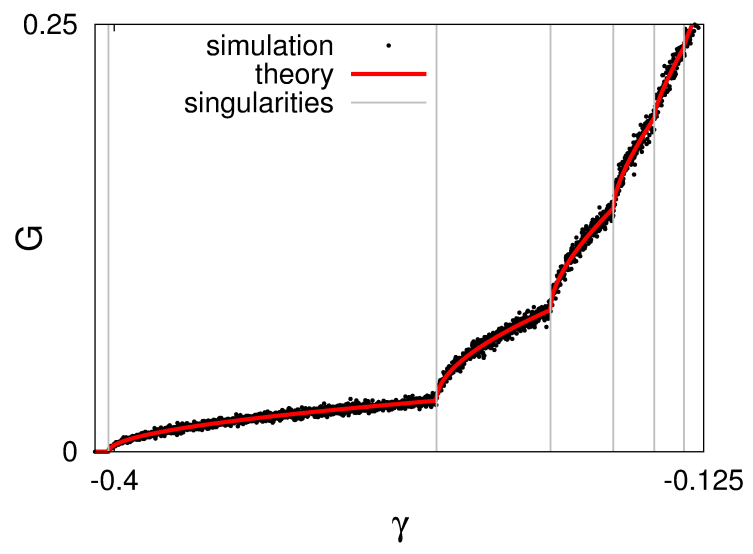

(c)

Figure 3.7: Zooms in Fig. 3.6(c). $G$ is a non-smooth function, showing corners at the same position of the singularities. The corners occur at strong singularities. More clearly visible corners in the curves of $G$ are associated to more pronounced "peaks" in the histograms of $\gamma$. 
We have seen that the PDF of $\gamma$, defined by function $g(\gamma)$ and which is the derivative fo $G(\gamma)$, has singularities near maxima and minima of $J_{0}$. Such singularities are descontinuities of $g(\gamma)$ and explain the non-smooth character of $G(\gamma)$, clearly seen in Figs. 3.7(a)-(c), that correspond to zooms in three different regions of Fig. 3.6(c). The singularities are indicated by vertical lines (in gray color).

Figures 3.7(a)-(c) show corners located at the same position of the singularities. However, not all singularities have clearly visible corners associated to them. In Fig. 3.7(b), though there are many singularities near $\gamma=0, G$ is quite smooth near them. The figures indicate that visible corners appear only in case of strong singularities. As mentioned before, strong singularities are associated to peaks in the histograms of $\gamma$. If the derivative of $G$, given by $g$, has a singularity, $G$ has a corresponding corner, but this corner is not necessarily visible. Weak singularities do not produce visible corners.

\subsection{Probability of Global Chaos $\left(P_{c}\right)$}

In this section, we obtain the probability of global chaos, i.e. the probability of finding a particle moving in a phase space with no Kolmogorov-Arnold-Moser (KAM) barriers. Since $\gamma=K / K_{0}$, the transition to global chaos occurs if:

$$
|\gamma|>K_{c} / K_{0}
$$

where $K_{c}$ is the critical parameter that defines the transition to global chaos in the standard map. According to [37], $K_{c} \simeq 0.9716$. As discussed in chapter 2 , increasing $K_{0}$ and keeping constant $\hat{\rho}$ increase the effective perturbation parameter $K$ and the amount of chaos in phase space, i.e. the area of the phase space occupied by chaotic orbits.

Assuming here just positive values for $K_{0}$, the probability of global chaos, $P_{c}$, is given by:

$$
P_{c}=1-P\left(-K_{c} / K_{0} \leq \gamma \leq+K_{c} / K_{0}\right)
$$

where $P\left(-K_{c} / K_{0} \leq \gamma \leq+K_{c} / K_{0}\right)$ measures the probability of $\gamma$ belonging to the interval $-K_{c} / K_{0} \leq \gamma \leq+K_{c} / K_{0}$ or, equivalently, the rate of particles moving in phase spaces with KAM barriers. Thus, $P_{c}$ measures the portion of particles moving in global chaos phase spaces. 
Equation (3.46) can be rewritten as:

$$
P_{c}=1-\left[G\left(+K_{c} / K_{0}\right)-G\left(-K_{c} / K_{0}\right)\right]
$$

Considering that $K_{c} / K_{0}>0$ and substituting (3.44) in (3.47), there are three possible expressions for the probability of global chaos:

- If $K_{c} / K_{0} \geq \gamma_{\max }$, then $G\left(K_{c} / K_{0}\right)=1, G\left(-K_{c} / K_{0}\right)=0$, and:

$$
P_{c}=0, \quad \text { for } \quad 0<\frac{K_{0}}{K_{c}} \leq 1
$$

- If $\left|\gamma_{\min }\right| \leq K_{c} / K_{0}<\gamma_{\max }$, then:

$$
G\left(+K_{c} / K_{0}\right)=\sum_{\hat{\rho}_{i}^{+} \in \Gamma_{+K_{c} / K_{0}}}(-1)^{i} \exp \left[-\frac{1}{2}\left(\frac{\hat{\rho}_{i}^{+}}{\hat{\rho}_{t h}}\right)^{2}\right]
$$

$G\left(-K_{c} / K_{0}\right)=0$, and:

$$
P_{c}=1-\exp \left[-\frac{1}{2}\left(\frac{\hat{\rho}_{0}^{+}}{\hat{\rho}_{t h}}\right)^{2}\right], \quad \text { for } \quad 1<\frac{K_{0}}{K_{c}} \leq \frac{1}{\left|\gamma_{\min }\right|}
$$

since, in this interval, $\Gamma_{+K_{c} / K_{0}}$ has only one element, $\hat{\rho}_{0}^{+}$.

- If $0<K_{c} / K_{0}<\left|\gamma_{\min }\right|$, then Eq. (3.49) also applies for $G\left(+K_{c} / K_{0}\right), G\left(-K_{c} / K_{0}\right)$ is given by:

$$
G\left(-K_{c} / K_{0}\right)=\sum_{\hat{\rho}_{i}^{-} \in \Gamma_{-K_{c} / K_{0}}}(-1)^{i} \exp \left[-\frac{1}{2}\left(\frac{\hat{\rho}_{i}^{-}}{\hat{\rho}_{t h}}\right)^{2}\right]
$$

and:

$$
P_{c}=1-\left\{\sum_{\hat{\rho}_{i}^{+} \in \Gamma_{+K_{c} / K_{0}}}(-1)^{i} \exp \left[-\frac{1}{2}\left(\frac{\hat{\rho}_{i}^{+}}{\hat{\rho}_{t h}}\right)^{2}\right]-\sum_{\hat{\rho}_{i}^{-} \in \Gamma_{-K_{c} / K_{0}}}(-1)^{i} \exp \left[-\frac{1}{2}\left(\frac{\hat{\rho}_{i}^{-}}{\hat{\rho}_{t h}}\right)^{2}\right]\right\}
$$

where $\frac{K_{0}}{K_{c}}>\frac{1}{\left|\gamma_{\min }\right|}$.

Let $S_{K_{c} / K_{0}}$ be the ordered set $\left(\hat{\rho}_{0}^{+}, \hat{\rho}_{0}^{-}, \hat{\rho}_{1}^{-}, \hat{\rho}_{1}^{+}, \hat{\rho}_{2}^{+}, \hat{\rho}_{2}^{-}, \hat{\rho}_{3}^{-} \ldots\right)$, which corresponds to set formed by the elements of $\Gamma_{+K_{c} / K_{0}}$ and $\Gamma_{+K_{c} / K_{0}}$. Denoting the terms of $S$ by $\hat{\rho}_{i}$ such that 
$\hat{\rho}_{0}=\hat{\rho}_{0}^{+}, \hat{\rho}_{1}=\hat{\rho}_{0}^{-}, \hat{\rho}_{2}=\hat{\rho}_{1}^{-}$, and so on, Eqs (3.48), (3.50) and (3.52) can be written in a more compact form:

$$
P_{c}=\left\{\begin{array}{cc}
0 & 0<\frac{K_{0}}{K_{c}} \leq 1 \\
1-\sum_{\hat{\rho}_{i} \in S_{K_{c} / K_{0}}}(-1)^{i} \exp \left[-\frac{1}{2}\left(\frac{\hat{\rho}_{i}}{\hat{\rho}_{t h}}\right)^{2}\right] & 1<\frac{K_{0}}{K_{c}}
\end{array}\right.
$$

We note that $S_{K_{c} / K_{0}}$ is also the set of solutions $\hat{\rho}_{i}$ of $K_{c} / K_{0}=\left|J_{0}\left(\hat{\rho}_{i}\right)\right|$, where $\left|J_{0}\left(\hat{\rho}_{i}\right)\right|$ corresponds to the absolute value of $J_{0}\left(\hat{\rho}_{i}\right)$.

In Figure 3.8, $P_{c}$ is plotted as a function of $\frac{K_{0}}{K_{c}}$ for different fixed values of $\hat{\rho}_{t h}$. For $0<K_{0} / K_{c} \leq 1, P_{c}=0$, which means that all particles move in phase spaces with KAM barriers. In this case, some of the particles are trapped inside stability islands and those following chaotic orbits remains confined in regions isolated by KAM barriers.

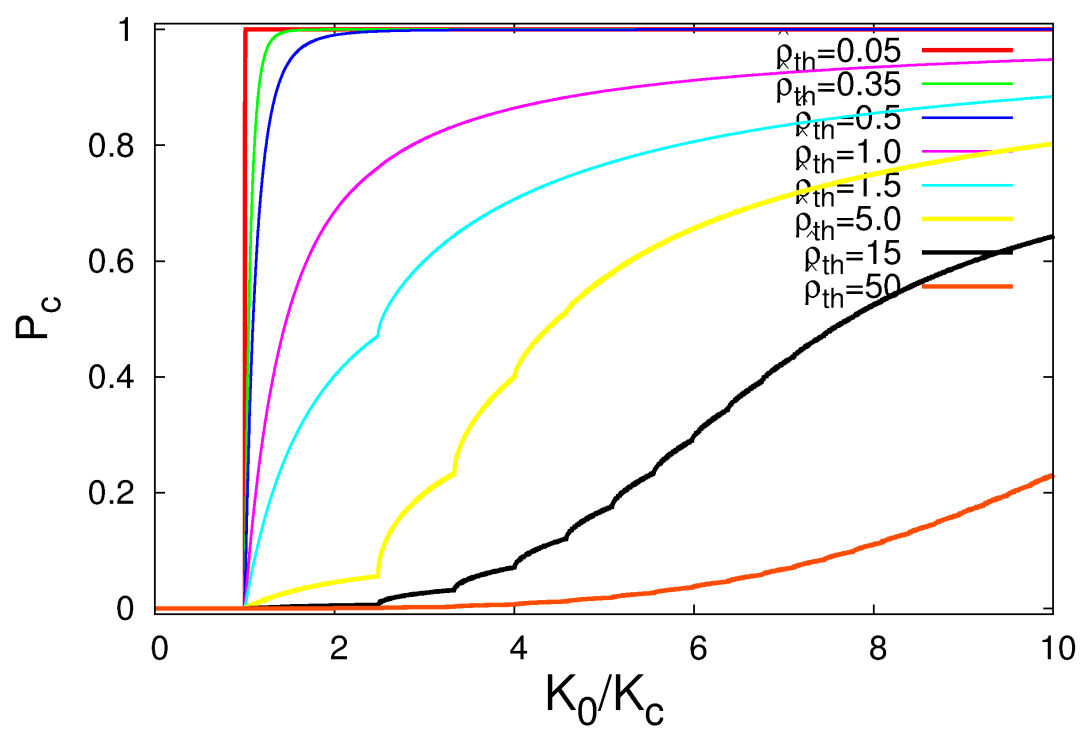

Figure 3.8: Probability of global chaos $\left(P_{c}\right)$ as a function of $K_{0} / K_{c}$ and for different values of $\hat{\rho}_{t h}$. For $0<K_{0} / K_{c} \leq 1, P_{c}=0$ : all particles are confined inside stability islands or chaotic regions isolated by KAM barriers. For high and increasing values of $K_{0} / K_{c}, P_{c}$ goes to one, that is, particles following chaotic orbits move freely in the radial direction since all KAM barriers are broken.

As can also be seen in Fig. 3.8, for $K_{0} / K_{c}>1$ and increasing $K_{0}, P_{c}$ goes from zero to one, that is, most particles move in phase spaces without KAM barriers and those following chaotic orbits move freely in the radial direction.

According to Eq. (3.53), for high values of $K_{0} / K_{c}$ (or small $K_{c} / K_{0}$ ) the sum in (3.53) goes to zero. That happens because consecutive elements of $S_{K_{c} / K_{0}}, \hat{\rho}_{i}$ and $\hat{\rho}_{i+1}$, become 


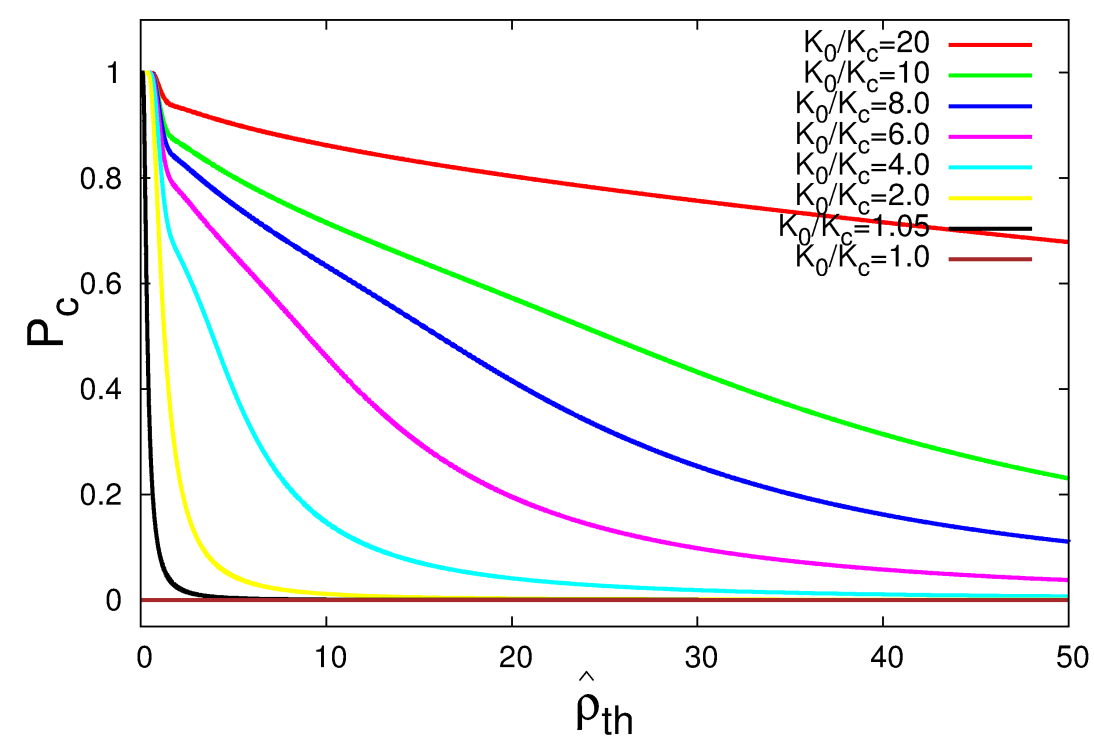

Figure 3.9: Probability of global chaos $\left(P_{c}\right)$ as a function of $\hat{\rho}_{t h}$ and for different fixed values of $K_{0} / K_{c}$.

both near one of the zeros of $\left|J_{0}(\hat{\rho})\right|$. Thus, the exponential terms of the sum, evaluated at $\hat{\rho}_{i}$ and $\hat{\rho}_{i+1}$ and that have oppposite signs, cancel each other.

The probability of global chaos is plotted also in Fig. 3.9 as a function of $\hat{\rho}_{t h}$ and for different fixed values of $K_{0} / K_{c}$. If $0<K_{0} / K_{c} \leq 1, P_{c}$ is always zero for any $\hat{\rho}_{t h}$, as indicated by the brown horizontal line $\left(K_{0} / K_{c}=1\right)$. If $K_{0} / K_{c}>1, P_{c}$ is close to one for small $\hat{\rho}_{t h}$ and decays for increasing $\hat{\rho}_{t h}$.

Still considering the case $K_{0} / K_{c}>1$, the plots shown in Fig. 3.9 also indicate a fast decreasing for small $\hat{\rho}_{t h}$ and a slower one for high $\hat{\rho}_{t h}$, what, according to Eq. (3.53), can explained considering that $d P_{c} / d \hat{\rho}_{t h} \sim 1 / \hat{\rho}_{t h}^{3}$.

\subsection{Escape Rate}

Motivated by the results of section 3.5, we now analyze a numerical transport measure called "escape rate" or, simply, $\eta_{e}$, Measures of the escape rate are presented for the standard map and also for the GSM. In the latter case, these measures are compared to the probability of global chaos

The escape rate can be computed by the following steps: 
- First, we define an ensemble of $N$ particles whose initial positions $\left(\theta_{0}, I_{0}\right)$ are spread over a line, defined by a fixed initial radial coordinate $I_{0}$. The initial poloidal coordinate $\theta_{0}$ is a random number between 0 and $2 \pi$ chosen according to a random uniform distribution.

- Second, for each particle of the ensemble, the map is iterated until the particle "escapes", what occurs when its radial coordinate, $I_{n}$, satisfies the escape condition $\left|I_{n}-I_{0}\right|>2 \pi$ for a number of iterations $n$ such that $n \leq T$, where $T$ corresponds to the maximum number of iterations allowed. If $n=T$ and the escape condition was not satisfied, we consider that the particle has not escaped. We then stop iterating the map and repeat the procedure for the other particles of the ensemble.

- Finally, after performing the previous step for all particles, we calculate the ratio $N_{e} / N$, where $N_{e}$ is the number of particles that have escaped.

In the procedure to compute $\eta_{e}$, it is necessary to specify the initial radial coordinate $I_{0}$. We adopt $I_{0}=\pi$, although any other position for the line of initial conditions is valid. We avoid using $I_{0}$ near zero because a significative number of particles can be located inside the period-one island, which can occupy a relatively large area, even in a global chaos phase space. In that case, it is necessary to wait the maximum number of iterations $T$, making the procedure computationally expensive.

The reason for the escape condition adopted is the following. The standard map's phase space is invariant by translations of $2 \pi$ in the direction of the $I$ coordinate, what means that objects like islands, quasi-periodic or chaotic orbits repeat themselves by translations of $2 \pi$. Thus, if a particle initially located at $I_{0}$ is found in a position $I_{n}$ such that the escape condition holds, it follows the same orbit in the next $n$ iterations and is always escaping from regions between the limits $I_{0} \pm 2 \pi m$ and $I_{0} \pm 2 \pi(m+1)$, where $m=1,2,3, \ldots$.

Figure 3.10 shows plots of $\eta_{e}$ versus $K / K_{c}$ for the standard map. $K$ is the perturbation parameter, and $K_{c}$ is the critical parameter that defines the transition to global chaos. All particles have the same $K$, and, as mentioned before, $K_{c} \simeq 1$.

The plots are created for different values of $T: T=1000,5000$, and 10000 iterations. 


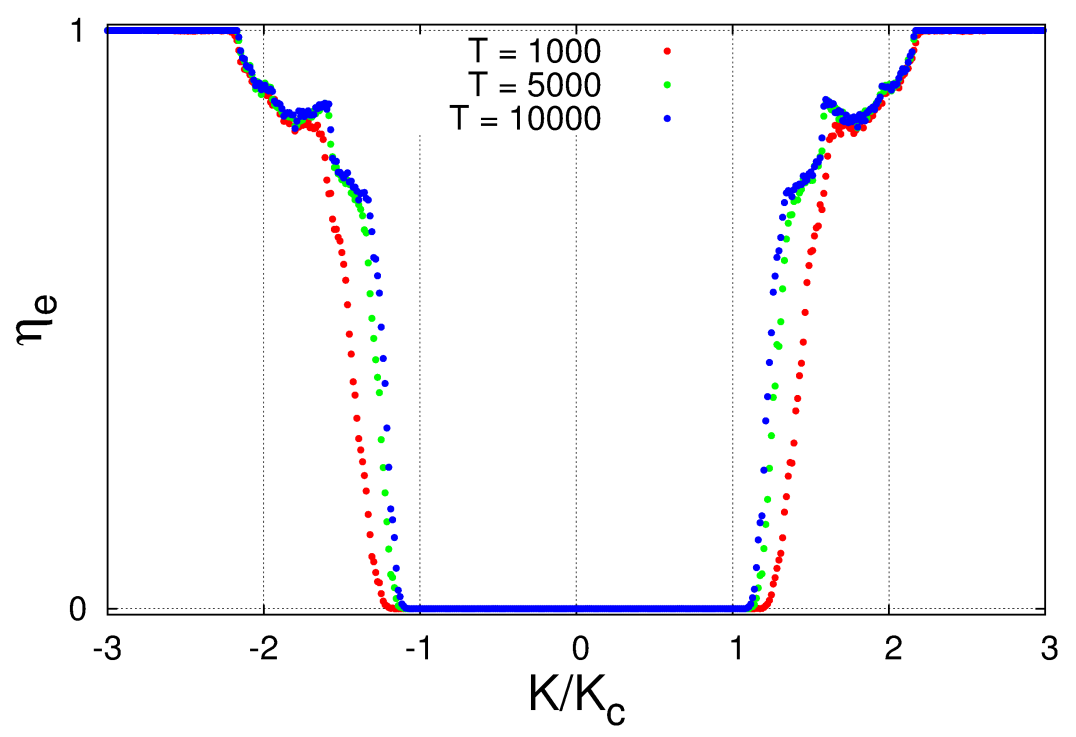

Figure 3.10: Rate of escaping particles, $\eta_{e}$, versus $K / K_{c}$ for the standard map model. $K$ is the perturbation parameter of the standard map, and $K_{c}$ is the critical parameter defining the transition to global chaos. If $K>K_{c}$, there are no KAM barriers, allowing particles that follow chaotic orbits to escape. Due to trapping effects around or inside stability islands, there is no abrubt transition in escape rate for $K \simeq K_{c}$.

Each point corresponds to a simulation where $\eta_{e}$ is calculated for an ensemble of $N=5000$ particles.

As can be seen in Fig. 3.10, $\eta_{e}=0$ for $|K| \leq K_{c}$. Due to the presence of KAM barriers, there are no particles escaping if the absoulte value of the perturbation is below the critical parameter. Particles trapped inside islands or following quasi-periodic orbits can not escape, just as those following chaotic orbits, which are confined among KAM barriers.

For $|K|>K_{c}$, Fig. 3.10 shows that $\eta_{e}=0$ increase with $|K|$. Since there are no KAM barriers, particles following chaotic orbits can escape. However, due to trapping of particles inside islands or long time stickiness of chaotic orbits near islands, there is not a well defined or an abrubt transition from zero to one at $|K|=K_{c}$. Higher perturbation values can destroy islands and increase the amount of chaos, bringing the escape rate to maximum value.

Figure 3.11 shows the GSM's escape rate (colored points) versus $K_{0} / K_{c}$ for different and fixed values of $\hat{\rho}_{t h}$. Each point corresponds to a simulation where $\eta_{e}$ is calculated 


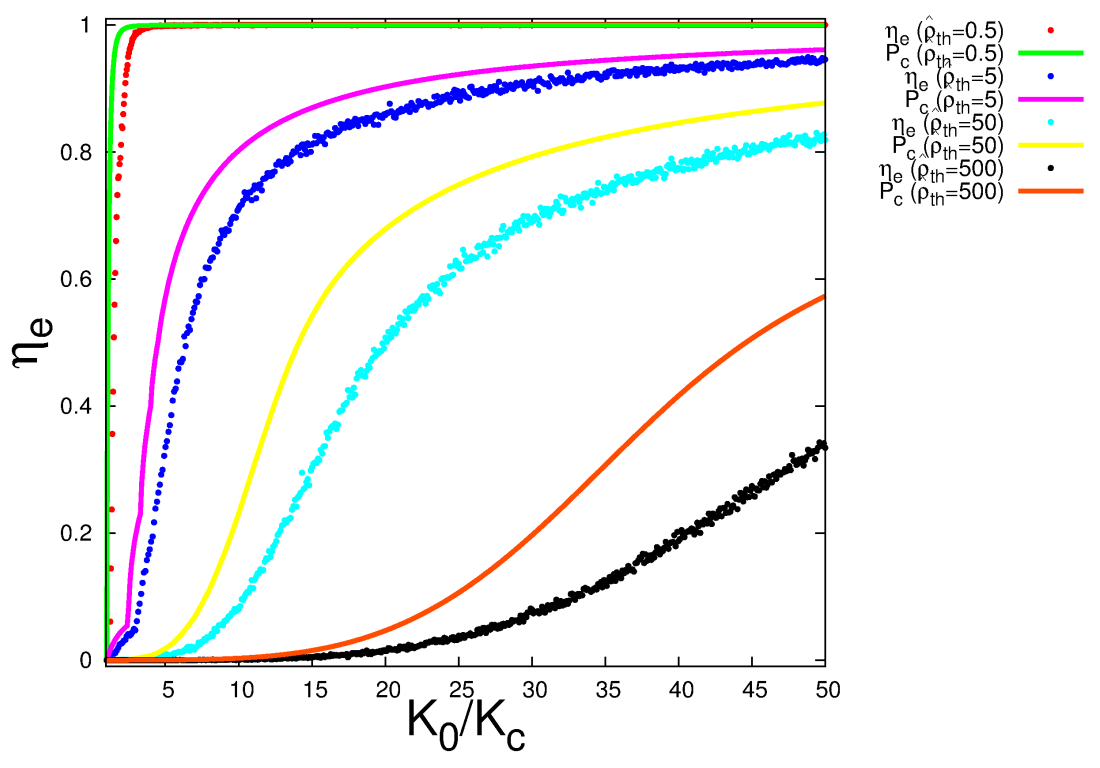

Figure 3.11: GSM model's escape rate (colored points) for increasing $K_{0} / K_{c}$ and fixed $\hat{\rho}_{t h}$ compared to the probability of global chaos (colored continuous lines). The analytical quantity $P_{c}$ provides an upper bound for $\eta_{e}$. Parameters: $N=5 \times 10^{3} ; T=5 \times 10^{3}$.

for an ensemble of $N=5 \times 10^{3}$ particles and a maximum number of iterations given by $T=5 \times 10^{3}$. The particles have different random Larmor radii, generated according to the PDF (3.22). Let us denote these random numbers as $\hat{\rho}_{l}$, where $l=1 . . N$. Orbits are computed by iterating $N$ gyroaveraged standard maps with different effective perturbation $K_{l}=K_{0} J_{0}\left(\hat{\rho}_{l}\right)$ and the same $K_{0}$ for all particles.

In Fig. 3.11, the escape rate is plotted with the probability of global chaos (colored continuous lines) using the same value for $\hat{\rho}_{t h}$. The results show that the analytical quantity $P_{c}$ provides an upper bound for $\eta_{e}$. Although $P_{c}$ measures the rate of orbits embedded in global chaos phase spaces, not all of them can escape. As already mentioned, there are trapping or stickiness effects due to the existence of islands, and these effects make the transport measure $\eta_{e}$ lesser than $P_{c}$.

However, the difference diminishes with increasing $K_{0}$. According to Eq. (3.32), the mean effective perturbation $\langle K\rangle$ increases proportionally with $K_{0}$, supressing islands' effects and increasing chaos.

Figure 3.12 shows plots of the GSM model's escape rate and probability of global chaos for varying $\hat{\rho}_{t h}$ and fixed $K_{0} / K_{c}$. Again, the escape rate is below the limit provided by the 


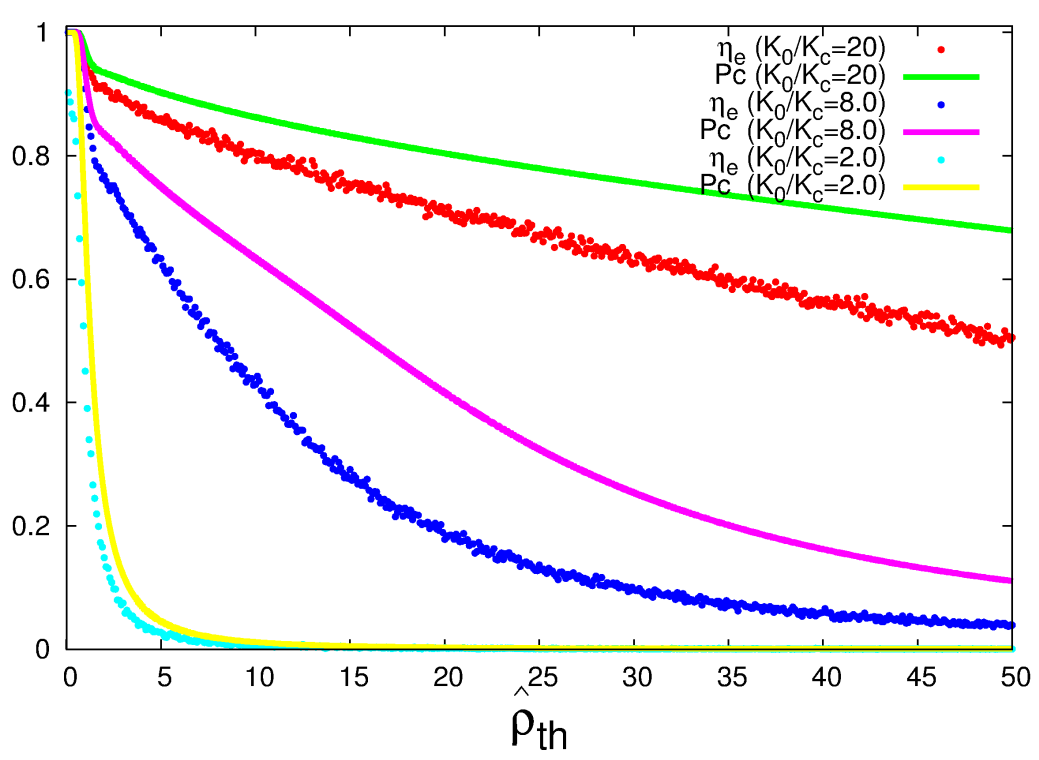

Figure 3.12: Rate of escaping particles and probability of global chaos in the GSM model for increasing $\hat{\rho}_{t h}$ and fixed $K_{0} / K_{c}$. Parameters: $N=5 \times 10^{3} ; T=5 \times 10^{3}$.

probability of global chaos. It is expected that both measures go to zero with increasing $\hat{\rho}_{t h}$. As prescribed by Eqs. (3.32) and (3.36), the average and dispersion of the effective perturbation go to zero for high values of $\hat{\rho}_{t h}$, restoring islands and KAM barriers. This is shown, for example, by the fast decaying plots of Fig. $3.12\left(K_{0} / K_{c}=2.0\right)$.

\subsection{Rate of Trapping by Period-One Islands}

In this section we define and analyze another numerical transport measure, which quantifies the amount of particles trapped by period-one islands. Period-one islands occupy the largest areas in the phase space of the standard map. We will call this measure rate of trapping and denote it by $\eta_{t}$.

In the GSM model, all particles move in phase spaces where the standard map's fixed point exists. Since the phase spaces' topologies are determined by different values of the effective perturbation, the fixed point can be stable or unstable, having then different effects on the transport of particles located near it.

Some particles "see" phase spaces where the fixed points are hyperbolic, and others where the fixed points are parabolic or elliptic. In general, particles located near el- 
liptic fixed points are trapped by the corresponding period-one islands, and those near hyperbolic points spread in their respective phase spaces.

Let $O$ be the standard map's fixed point located at $\theta=\pi$ and $I=0$. Given the translation invariance of the standard map, the discussion presented here can be applied to other fixed points located at $\theta=\pi$ and $I= \pm 2 \pi m$, where $m=1,2,3, \ldots$.

We compute the rate of trapping associated to $O$ using the following procedure:

- $N$ particles are randomly spread according to an uniform distribution inside a circle of center $O$ and radius $\epsilon r$, where $\epsilon \ll r$ and $r$ is the radius of other larger and concentric cicle.

- For a particle of the ensemble, the map is iterated until the particle escapes from the circle of radius $r$ or the number of iterations is greater than a maximum limit $T$. The particle is considered to be "trapped" if it remains inside the circle of radius $r$ during $T$ iterations. After deciding whether the particle is trapped, this step is repeated for the other particles.

- Finally, we calculate $\eta_{t}$, which is determined by the ratio $N_{t} / N$, where $N_{t}$ is the number of trapped particles.

In all simulations presented here, we adopt $\epsilon=0.05, r=1.0, N=5 \times 10^{3}$, and $T=5 \times 10^{3}$. Different values can also be used under the condition of keeping $\epsilon \ll r$, $r \leq \pi$ and using large values for $N$ and $T$. The condition $r \leq \pi$ comes from the fact that the standard map is modulated by $2 \pi$ in the direction of coordinate $\theta$.

Figure 3.13 shows measures of the trapping rate in the standard map (red points) versus $K / 4$, where $K$ denotes the perturbation parameter. For each measure, the orbits are computed by iterations of $N=5 \times 10^{3}$ standard maps with the same value for $K$.

There are two abrubt transitions occuring near 0 and $K_{c} \simeq 4.04$. During $T=5 \times 10^{3}$ iterations, orbits remain trapped $\left(\eta_{t}=1\right)$ for $0<K<K_{c}$. If $K<0$ or $K>K_{c}$, all of them escape $\left(\eta_{t}=1\right)$. The interval $0<K<K_{c}$, that we call here as trapping interval, corresponds approximately to the stability interval of the fixed point $O, 0<K<4$. $O$ is elliptic for $K$ inside the stability interval and hyperbolic otherwise. 


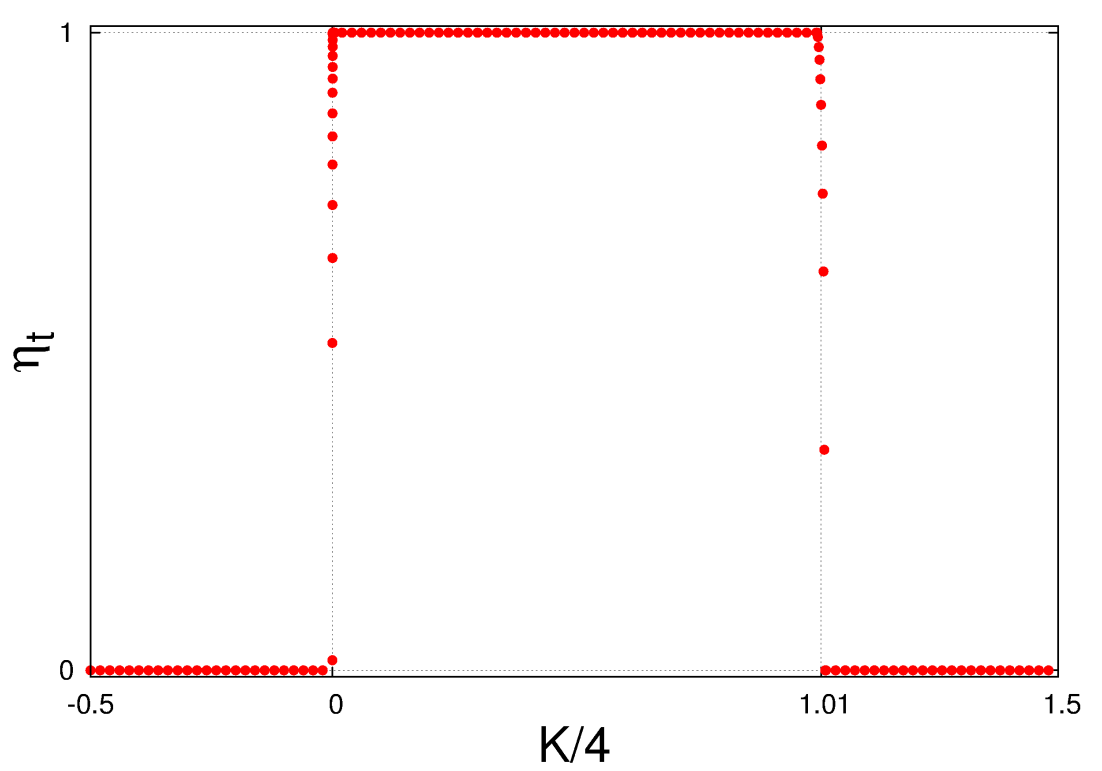

Figure 3.13: Rate of trapping in the standard map by the period-one island associated to the fixed point located at $\theta_{0}=\pi$ and $I_{0}=0$. Two abrubt transitions occur near 0 and $K_{c} \simeq 4.04$. The trapping interval $0<K<K_{c}$ corresponds approximately to the stability interval of the fixed point, which is elliptic for $0<K<4$ and hyperbolic for $K<0$ and $K>4$.

Before analyzing the rate of trapping in the GSM model, we define other analytical measure based on the cummulative distribution function of $\gamma$. We call this measure probability of trapping, or simply $P_{t}$, which is the probability of finding the particle moving in a phase space for which the effective perturbation $K$ is inside the trapping interval $0<K<K_{c}$

Assuming that $K_{0}>0$ and since $\gamma=K / K_{0}, P_{t}$ is then given by:

$$
P_{t}=P\left(0<\gamma<K_{c} / K_{0}\right)
$$

where $P$ denotes the probability of $\gamma$ inside the interval $0<\gamma<K_{c} / K_{0}$. Thus,

$$
P_{t}=G\left(K_{c} / K_{0}\right)-G(0)
$$

Applying (3.44), Eq. we have:

$$
P_{t}=1-\sum_{\hat{\rho}_{j} \in \Gamma_{0}}(-1)^{i} \exp \left[-\frac{1}{2}\left(\frac{\hat{\rho}_{j}}{\hat{\rho}_{t h}}\right)^{2}\right], \quad \text { for } \quad 0<K_{0} / K_{c} \leq 1
$$




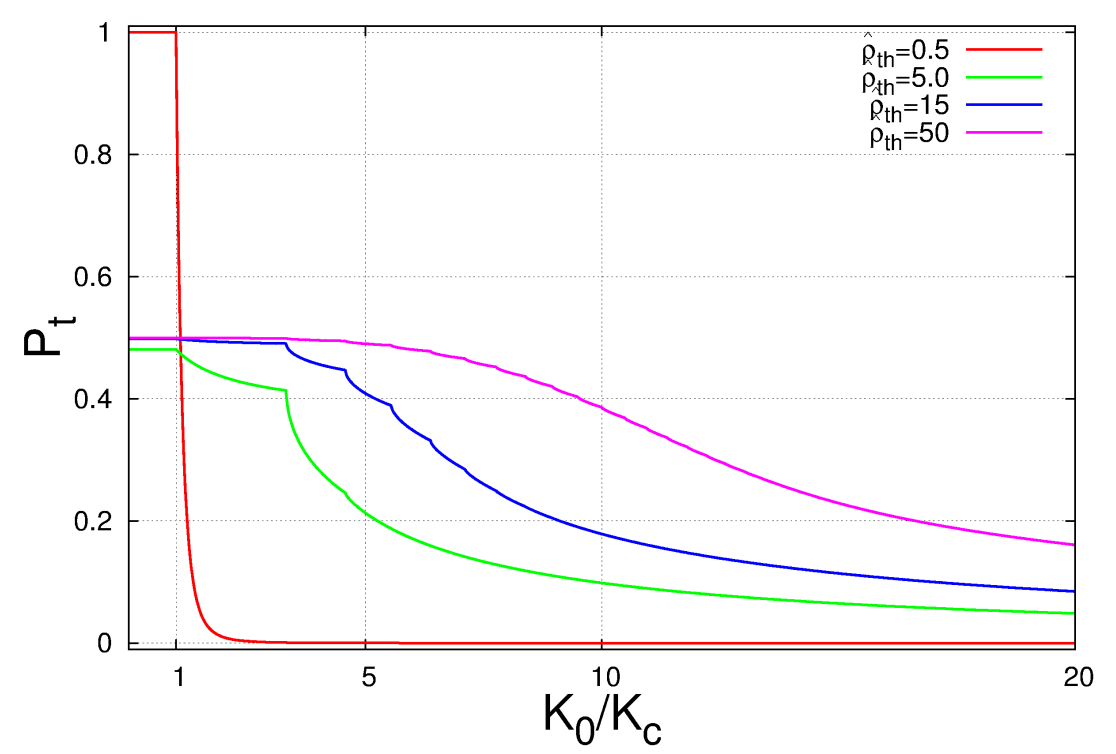

Figure 3.14: Probability of trapping, $P_{t}$, versus $K_{0} / K_{c}$. $P_{t}$ is constant for $K_{0} / K_{c}<1$ and goes to zero for $K_{0} / K_{c} \gg 1$.

and:

$P_{t}=\sum_{\hat{\rho}_{i} \in \Gamma_{K_{c}} / K_{0}}(-1)^{i} \exp \left[-\frac{1}{2}\left(\frac{\hat{\rho}_{i}}{\hat{\rho}_{t h}}\right)^{2}\right]-\sum_{\hat{\rho}_{j} \in \Gamma_{0}}(-1)^{j} \exp \left[-\frac{1}{2}\left(\frac{\hat{\rho}_{j}}{\hat{\rho}_{t h}}\right)^{2}\right]$, for $K_{0} / K_{c}>1$

Figure 3.14 shows plots of the probability of trapping versus $K_{0} / K_{c}$ for different values of $\hat{\rho}_{t h}$. If $K_{0} / K_{c}<1, P_{t}$ is constant, what is in agreement with the fact that Eq. (3.56) has no dependence on $K_{0}$. If $K_{0} / K_{c}>1, P_{t}$ decreases with increasing $K_{0}$. According to Eq. (3.57), when $K_{0} / K_{c} \gg 1, \Gamma_{K_{c} / K_{0}} \rightarrow \Gamma_{0}$, i.e. the two sets become nearly equivalent and the corresponding sums cancel each other. Thus, $P_{t}$ goes to zero for high values of $K_{0} / K_{c}$

Figure 3.15 shows plots of the probability of trapping versus $\hat{\rho}_{t h}$ for different values of $K_{0} / K_{c}$. If $K_{0} / K_{c}=0.5, P_{t} \simeq 1$ for small values of $\hat{\rho}_{t h}$, decreases to a minimum and increases a constant level near 0.5. If $K_{0} / K_{c}>1$, the plots exhibit the following features: $P_{t}$ increases from zero to a maximum, decreases to a local minimum and increases again until reaching again the same level near 0.5.

The limit case of small $\hat{\rho}_{t h}$ values can be understood through the fast exponential decaying terms of Eqs. (3.56) and (3.57). If $\hat{\rho}_{t h} \rightarrow 0$, these terms go to zero and then we 


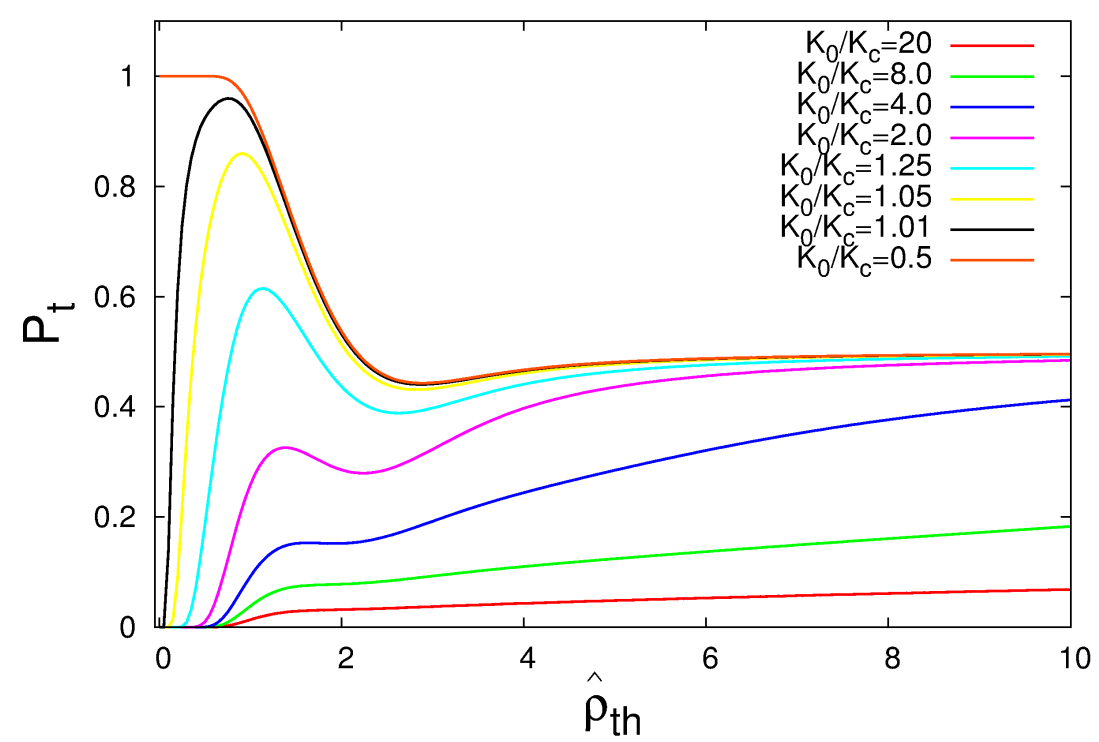

Figure 3.15: Probability of trapping $\left(P_{t}\right)$ versus $\hat{\rho}_{t h}$ for different values of $K_{0} / K_{c}$.

have: $P_{t} \rightarrow 1$ for $0<K_{0} / K_{c} \leq 1$ and $P_{t} \rightarrow 0$ for $K_{0} / K_{c}>1$.

As already mentioned, the plots in Fig. 3.15 show that $P_{t}$ becomes constant at high $\hat{\rho}_{t h}$. This is in agreement with Eqs. (3.56) and (3.57) since their derivatives regarding $\hat{\rho}_{t h}$ vary both like $d P_{t} / d \hat{\rho}_{t h} \sim 1 / \hat{\rho}_{t h}^{3}$, going to zero at high $\hat{\rho}_{t h}$.

Now we show, in Figs. 3.16 and 3.17, numerical results (colored points) refering to the rate of trapping in the GSM model. Each measure is computed for an ensemble of $N=5 \times 10^{3}$ particles with different random Larmor radii, determined by the PDF (3.22).

$N$ gyroaveraged standard maps, with different effective perturbation parameters and the same $K_{0}$, are iterated at most $T=5 \times 10^{3}$ times. The value of $K_{0}$ is defined by the product between a given ratio $K_{0} / K_{c}$, whose values are shown in the horizontal axis, and the critical parameter $K_{c}$, estimated as $K_{c} \simeq 4.04$.

The initial positions of the particles are randomly located near point $O$ inside a circular region of radius $\epsilon r$. Again, we adopt $\epsilon=0.05$, and the trapping circular region has a radius defined by $r=1$.

The plots of the rate of trapping in the GSM are compared to plots of the probability of trapping, the same shown in Figs. 3.14 and 3.15. We see that, in both Figs. 3.16 and 3.17, the analytical measures describe well the numerical ones. 


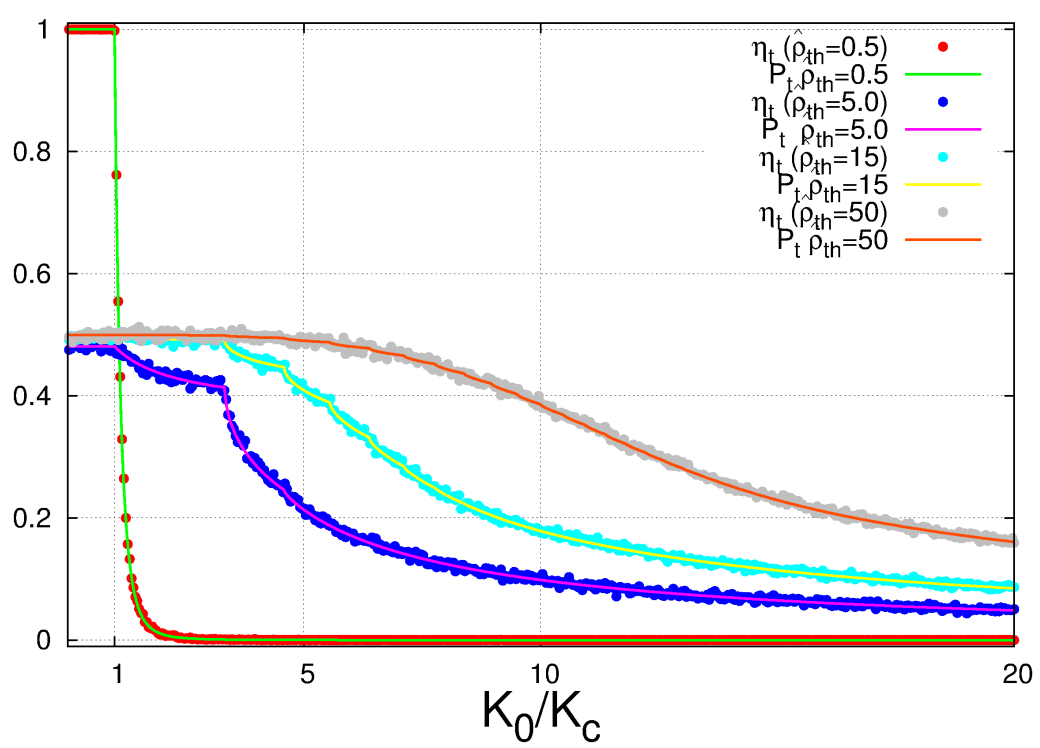

Figure 3.16: GSM's rate of trapping (points) versus $K_{0} / K_{c}$ for fixed values of $\hat{\rho}_{t h}$. The probability of trapping (lines), defined by Eqs. (3.56) and (3.57), provides a good analytical estimate for $\eta_{t}$. Parameters: $N=5 \times 10^{3} ; T=5 \times 10^{3} ; \epsilon=0.05 ; r=1$.

Some properties of the rate of trapping shown in Fig. 3.16 can be understood analyzing the average of the effective perturbation, $\langle K\rangle$. According to Eq. (3.32), for any $\hat{\rho}_{t h}>0$, $\langle K\rangle / K_{0} \leq 1$ and, if $\hat{\rho}_{t h}$ is kept fixed, $\langle K\rangle=O\left(K_{0}\right)$. Thus, $0<K_{0}<K_{c}$ results that $0<\langle K\rangle<K_{c}$, and particles, in average, are trapped by the preriod-one island. This explains why, even varying $K_{0}$ inside the trapping interval $0<K_{0}<K_{c}$, the rate of trapping remains approximately constant. However, increasing $K_{0}$ indefinitely moves the average of the effective perturbation outside the trapping interval in order that the rate of trapping starts to decay if $K_{0}>K_{c}$, as can also be seen in Fig. 3.16.

Figure 3.17 shows that the rate of trapping varies in two different ways for small $\hat{\rho}_{t h}$ : one for $0<K_{0}<K_{c}$ and other for $K_{0}>K_{c}$.

Let us consider the first case, illustrated by the set of measures of $\eta_{t}$ for $K_{0}=0.5 K_{c}$ (red points). According to Eqs. (3.33) and (3.37), if $\hat{\rho}_{t h} \rightarrow 0$, then $\langle K\rangle \rightarrow K_{0}, \sigma_{K}^{2} \rightarrow 0$ and, since $0<K_{0}<K_{c}$, most particles are trapped. This property is observed in Fig. 3.17, where $\eta_{t} \simeq 1$ for small $\hat{\rho}_{t h}$ and $K_{0}=0.5 K_{c}$.

In the cases where $K_{0}>K_{c}$, we have again $\langle K\rangle \rightarrow K_{0}$ and $\sigma_{K}^{2} \rightarrow 0$ for $\hat{\rho}_{t h} \rightarrow 0$, but 


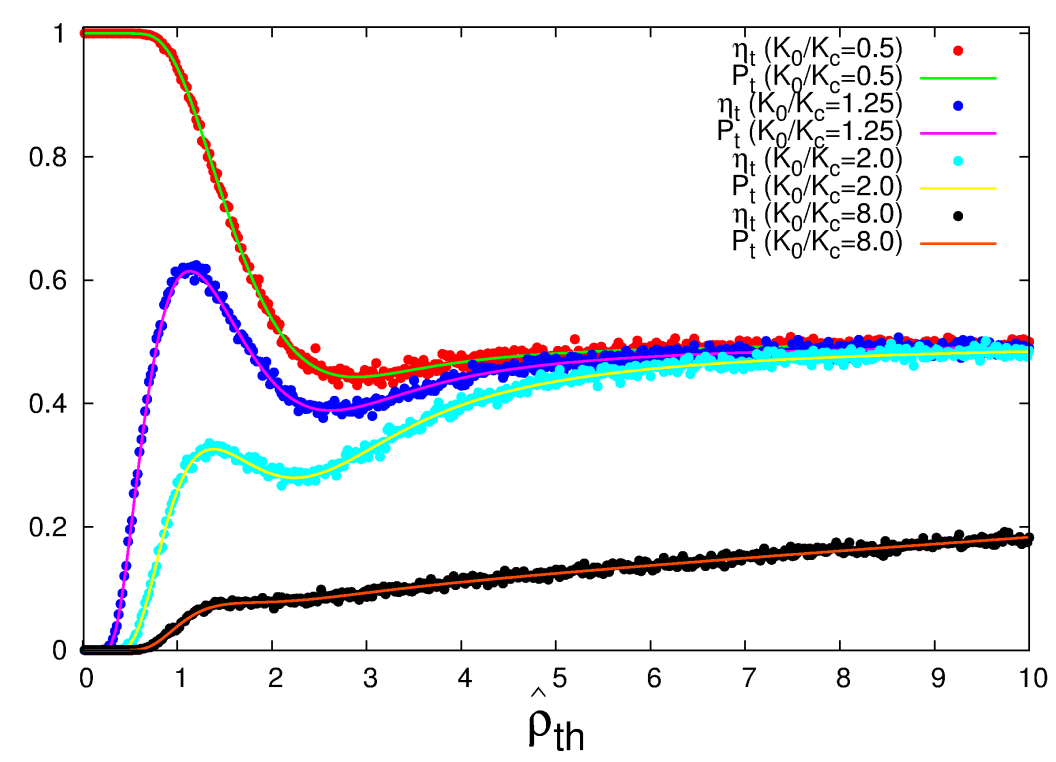

Figure 3.17: $\eta_{t}$ (points) versus $\hat{\rho}_{t h}$ for different fixed values of $K_{0} / K_{c} . \eta_{t}$ is compared to $P_{t}$ (lines). Both results are in good agreement. Parameters: $N=5 \times 10^{3} ; T=5 \times 10^{3} ; \epsilon=0.05 ; r=1$.

most $K$ values are outside the trapping interval. Thus, $\eta_{t} \simeq 0$ for small $\hat{\rho}_{t h}$ and $K_{0}>K_{c}$. However, since $\langle K\rangle \sim \exp \left(-\hat{\rho}_{t h}^{2} / 2\right)$, increasing $\hat{\rho}_{t h}$ moves $\langle K\rangle$ inside the trapping interval, increasing then the number of trapped particles.

In all cases shown in Fig. 3.17, we observe the ocurrence of local minima in the rate of trapping. This property can be explained by the way how the dispersion of the effective perturbation varies with increasing $\hat{\rho}_{t h}$. We have seen that, according to Eq. $(3.37),\langle K\rangle$, keeping $K_{0}$ fixed, varies in the same way as $\sigma_{\gamma}^{2}$, increasing from zero until reaching a maximum and then decreasing to zero again.

If $\langle K\rangle$ is inside the trapping interval and the dispersion increases, then the number of values of the effective perturbation outside the trapping interval also increases. If the dispersion reaches its maximum, then the rate of trapping reaches a minimum.

Since $\langle K\rangle$ remains inside the trapping interval $\left(\langle K\rangle \rightarrow 0^{+}\right.$for $\left.\hat{\rho}_{t h} \rightarrow+\infty\right)$, if the dispersion starts to decrease from its maximum, then values of the effective perturbation are brought back to the trapping interval and then the rate of trapping starts to increase.

A final comment about Fig. 3.17 refers to the constant level $\eta_{t} \simeq 0.5$, reached at high values of $\hat{\rho}_{t h}$. Let $I_{\epsilon}$ be a small neighborhood of zero, defined by $I_{\epsilon}=(-\epsilon,+\epsilon)$ and such 
that $\epsilon$ is positive and arbitrarily small. The probability that $\gamma$ is positive given that $\gamma \in I_{\epsilon}$ is the conditional probability $P_{+}=P(0<\gamma<+\epsilon \mid-\epsilon<\gamma<+\epsilon)$, which can be written as:

$$
P_{+}=\frac{G(\epsilon)-G(0)}{G(\epsilon)-G(-\epsilon)} .
$$

Since $G( \pm \epsilon)=G(0) \pm \epsilon g(0)+O\left(\epsilon^{2}\right)$, we see that $P_{+}=\epsilon g(0) / 2 \epsilon g(0)=1 / 2$. Therefore, if $\gamma$ has values near zero, these values occur with equal probabilities inside $-\epsilon<\gamma<0$ and $0<\gamma<+\epsilon$.

For $\hat{\rho}_{t h} \rightarrow+\infty,\langle\gamma\rangle \rightarrow 0, \sigma_{\gamma}^{2} \rightarrow 0$, resulting that values of $\gamma$ become concentrated in a small neighborhood $I_{\epsilon}$, half of them in $(-\epsilon, 0)$ and the other half in $(0,+\epsilon)$. Thus, increasing $\hat{\rho}_{t h}$ makes values of the effective perturbation $K$ to concentrate inside $\left(-\epsilon K_{0},+\epsilon K_{0}\right)$, half outside the trapping interval $\left(-\epsilon K_{0}<K<0\right)$ and the other half inside the trapping interval $\left(0<K<+\epsilon K_{0}<<K_{c}\right)$. This means that $\eta_{t}$ goes to $1 / 2$ for high $\hat{\rho}_{t h}$, as shown in Fig. 3.17. 


\section{Chapter 4}

\section{Conclusion}

In this work we studied several finite Larmor radius effects on discrete models of $\vec{E} \times \vec{B}$ transport of test charged particles. FLR effects were included in our model by gyroaveraging the electrostatic potential. The discrete models correspond to area preserving maps assuming an eletrostatic potential consisting of a superposition of an equilibrium field and a perturbation consisting of a broad spectrum of drift waves. The maps were constructed from the integration of the gyro-averaged $\vec{E} \times \vec{B}$ Hamiltonian system, whose frequency is determined by the equilibrium potential. For zero Lamor radius (i.e., in the absence of FLR corrections), the $\vec{E} \times \vec{B}$ frequency's profile corresponds to the radial component of the eletric field.

We considered three different equilibrium potentials and constructed three different maps: the gyro-averaged standard map (GSM), the gyro-averaged standard nontwist map (GSNM), and the gyro-averaged quartic nontwist map (GQNM). In the GSM, the frequency, i.e. the gyro-averaged $\vec{E} \times \vec{B}$ zonal flow, has a monotonic radial profile. The GSNM and GQNM are nontwist maps their frequencies have non-monotonic radial profiles. Non-monotonic frequencies have degenerate points (points where the frequency is maximum or minimum) which cause the occurence of robust transport barriers, that we called nontwist transport barriers (NTBs). A NTB is a region of the phase space formed by KAM curves that are very resiliant to breakup. The KAM curves, in the context of our $\vec{E} \times \vec{B}$ models, preclude the radial transport of charged particles.

The perturbation parameter in all maps is proportional to the amplitude of the drift 
waves. Increasing the perturbation increases the chaos in the phase space. However, even for a high perturbation, we showed that, when the Larmor radius is sufficiently close to the zeros of the zero-order Bessel function, chaotic dynamics can be significantly supressed. Chaos suppression means that KAM curves are restored, forming barriers that restrict the transport of charged particles. The chaos supression effect due to the zeros of the zero-order Bessel function was analytically verified in our simplest model, the GSM, by applying the well-known critical threshold for the transition to global chaos in the Chirikov-Taylor map. We studied numerically the same effect in the GSNM by using breakup diagrams.

The breakup diagrams provide critical thresholds for the breakup of the shearless curve, one of the robust KAM curves that constitute the NTB. We used a method to determine the breakup of the shearless curve based on the maximum number of different recurrence times. We showed that a relatively low number of iterations was sufficient to determine the main features of the GSNM's breakup diagrams. As far as we know, few works have applied the recurrence properties of orbits as a tool to recognize chaotic motion.

We also used the GSNM to investigate the role of the Larmor radius in the formation of typical nontwist phase space topologies. The phase space of the GSNM exhibits separatrix reconnection, homoclinic and heteroclinic topologies. We obtained a formula determining the condition for separatrix reconnection as a function of the Larmor radius. In particular, by changing the Larmor radius, the phase space can assume the homoclinic or heteroclinic topologies.

Fixed points play an important role on the transport properties of dynamical systems. In the GSNM, the location and stability of the fixed points are affected by the Larmor radius. In order to determine the stability of the GSNM's fixed points, we applied Greene's residue criterion and computed the residues of the fixed points. The residue of each fixed point is a function of all the parameters of the GSNM, including the Larmor radius. The residues are correlated in such a way that knowing the residue of only one fixed point is sufficient to determine the residues of the others. As a consequence, we showed that there are only three possible configurations describing the fixed points in terms of their 
positions and stability.

For the GQNM, the other nontwist map studied in this work, we analysed the FLR effect on zonal flow bifurcation. The zonal flow bifucation occurs when the Larmor radius exceeds a critical threshold, and we showed that it is followed by the formation of additional fixed points and NTBs. In particular, we found a set of eight period-one fixed points and verified that four of them only exist for Larmor radii greater than a critical threshold. It was noticed that the elliptic fixed points created by varying the Larmor radius can acquire a strong stability, i.e. high perturbations are required for the elliptic points to become hyperbolic. The presence of elliptic points results in the formation of islands that trap charged particles. If the elliptic points have a strong stability, the associated islands cannot be easily destroyed by increasing the perturbation parameter. A consequence of the zonal flow bifurcation is the occurrence of three nontwist transport barriers. We focused on the central NTB and analysed the robustness of its shearless curve through breakup diagrams. The results show that the robustness of the central shearless significantly increases when the Larmor radius is close to the critical threshold for the zonal flow bifurcation. In this work we argued that, near the critical threshold, a high level of "flatness" of the frequency profile around the central degenerate point is associated with a high level of robustness of the central shearless curve. This property, which is in agreement with previous works, results in the formation of stronger NTBs near the threshold of the zonal flow bifurcation.

After studying FLR effects on the dynamics of single particles, we proposed a model based on the GSM to investigate statistical properties of ensembles of particles. In this GSM based model, we considered particles that "observe" different phase space topologies. Each particle of the ensemble has a random Larmor radius and follows a GSM orbit for which the effective perturbation is also randomly defined.

The Larmor radius' probability density function (pdf) results from the MaxwellBolztmann distribution, which describes plasmas in thermal equilibrium. The only parameter present in the Larmor radius' pdf is the thermal Larmor radius, which incorporates important physical parameters and determines the mean Larmor radius of an ensemble of particles. 
Having in mind the goal of providing results relevant to transport studies, here we focused on statistical properties related to the effective perturbation for it completely determines the orbit of each particle in the GSM model. The effective perturbation is the product of a constant, the perturbation parameter, and the zero-order Bessel function of Larmor radius, here called simply as gamma.

A first set of results refers to the statistics of the gamma function. We obtained its probability density function (pdf), average, dispersion and cumulative distribution function.

We showed the occurrence of high probability points or "peaks" in the histograms of gamma and related these peaks to singularity points in the gamma's pdf. The singularities correspond to maxima and minima of the zero-order Bessel function, defined at Larmor radii values which are zeros of the first-order Bessel function.

Although the peaks are always located near the singularities, not all singularities have peaks associated to them. The gamma's pdf has terms with exponential decaying factors coming from the Larmor radius' pdf and which are able to inhibit the effects of the singularities. The singularities can be "strong" (have peaks) or "weak" (have not peaks) depending on the magnitude of these factors at the singularities.

Among the properties of the average and dispersion of gamma, we saw that, for increasing Larmor radii, the average decays exponentially fast and the dispersion increases from zero to a maximum and then decreases to zero again. We argued that this property can also be explained by the infinite number of singularities concentrated near zero which become strong for high thermal Larmor radii.

An analytical formula for the cumulative distribution function (cdf) of gamma was obtained and validated by numerical simulations. We showed that this cdf is not a smooth function, presenting corners due to the singularities of the gamma's pdf. Our interest in the cdf of gamma comes from the fact that it allows to obtain formulas for probabilities associated to any specific interval of the effective perturbation

The examples discussed in this work were the probability of global chaos and the probability of trapping. The probabilities of global chaos and trapping were both analyzed considering variations of the perturbation parameter and the thermal Larmor radius. Even 
though evaluating the formulas of these probabilities require complicated computations, where it is necessary to solve transcedental equations involving the zero-order Bessel function, they showed to be useful tools for the study of two types of transport measures: the escape rate and the rate of trapping by period-one island.

The numerical results showed that the probability of global chaos represents an upper bound for the escape rate. The difference between both measures occurs because the escape rate in the standard map has not a well-defined transition at the critical parameter that defines the transition to global chaos. Many orbits, even in global chaos phase spaces, can be trapped inside or stick near outside stability islands. Despite the difference, which reduces for high values of the perturbation parameter, the escape rate can not be greater than the probability of global chaos. A particle can escape only in a global chaos phase space, but not all of the particles moving in global chaos phase spaces can do it.

We showed that the probability of trapping is a good theoretical estimate for the rate of trapping in the GSM model. This is due to a well-defined transition in the standard map' rate of trapping near the extrema of the fixed point's stability interval. Thus, in the context of GSM model, this well-defined transition makes the rate of trapping more strongly related to the statistics of the effective perturbation.

The analytical results presented here to describe statistical properties of the GSM model can also be applied to other types of transport measures, as, for example, to diffusion coefficients, often studied both analytically and numerically in previous works about the standard map. Combining known transport properties of the standard map, including those usually categorized as "strange" or "anomalous", and the results obtained here, we can study further transport properties of the GSM model. The work presented in Ref. [49] is an interesting starting point for future investigations in this direction.

Other possible direction consists in applying to more complicated gyro-averaged $\mathbf{E} \times \mathbf{B}$ models studied in previous works (see, for example, $[9,10]$ ) the same approach adopted here of combining statistical information about the most relevant parameters and nonlinear dynamics properties related to those models. 
Appendices 



\section{Appendix A}

\section{Gyro-averaged Drift Wave Map}

In this appendix, we show some details related to the obtaining process of the gyroaveraged drift wave map, given by Eqs. (2.11) and (2.12). The gyro-averaged drift wave map is the basis of all maps discussed in chapter 2 .

The gyro-average operation is defined by:

$$
\langle\phi(x, y, t)\rangle_{\varphi}=\frac{1}{2 \pi} \int_{0}^{2 \pi} \phi(x+\rho \cos \varphi, y+\rho \sin \varphi, t) d \varphi,
$$

We assumed an electrostatic potential of the form:

$$
\phi(x, y, t)=\phi_{0}(x)+A \sum_{m=-\infty}^{+\infty} \cos \left(k y-m \omega_{0} t\right) .
$$

Applying (A.1) to (A.2) results in:

$$
\langle\phi(x, y, t)\rangle_{\varphi}=\left\langle\phi_{0}(x)\right\rangle_{\varphi}+A \sum_{m=-\infty}^{+\infty}\left\langle\cos \left(k y-m \omega_{0} t\right)\right\rangle_{\varphi} .
$$

The terms $\left\langle\cos \left(k y-m \omega_{0} t\right)\right\rangle_{\varphi}$ can be solved as follows:

$$
\begin{aligned}
\left\langle\cos \left(k y-m \omega_{0} t\right)\right\rangle_{\varphi}= & \langle\cos (k y)\rangle_{\varphi} \cos \left(m \omega_{0} t\right)+\langle\sin (k y)\rangle_{\varphi} \sin \left(m \omega_{0} t\right) \\
= & \frac{\cos \left(m \omega_{0} t\right)}{2 \pi} \int_{0}^{2 \pi} \cos (k y+k \rho \sin \varphi) d \varphi+\frac{\sin \left(m \omega_{0} t\right)}{2 \pi} \int_{0}^{2 \pi} \sin (k y+k \rho \sin \varphi) d \varphi \\
= & \frac{\cos \left(m \omega_{0} t\right)}{2 \pi}\left\{\int_{0}^{2 \pi}[\cos (k y) \cos (k \rho \sin \varphi)-\sin (k y) \sin (k \rho \sin \varphi)] d \varphi\right\} \\
& +\frac{\sin \left(m \omega_{0} t\right)}{2 \pi}\left\{\int_{0}^{2 \pi}[\sin (k y) \cos (k \rho \sin \varphi)+\cos (k y) \sin (k \rho \sin \varphi)] d \varphi\right\} \\
= & J_{0}(k \rho)\left[\cos (k y) \cos \left(m \omega_{0} t\right)+\sin (k y) \sin \left(m \omega_{0} t\right)\right] \\
= & J_{0}(k \rho) \cos \left(k y-m \omega_{0} t\right)
\end{aligned}
$$


where $J_{0}$ is the zero-order Bessel function.

Substituting (A.4) in (A.3), we obtain:

$$
\langle H(x, y, t)\rangle_{\varphi}=\left\langle H_{0}(x)\right\rangle_{\varphi}+\frac{A}{B_{0}} J_{0}(\hat{\rho}) \sum_{m=-\infty}^{+\infty} \cos \left(k y-m \omega_{0} t\right),
$$

which is the gyro-averaged Hamiltonian introduced in chapter 2. The Hamiltonian A.5 can be rewritten as:

$$
\langle H(x, y, t)\rangle_{\varphi}=\left\langle H_{0}(x)\right\rangle_{\varphi}+\frac{A}{B_{0}} J_{0}(\hat{\rho}) \sum_{m=-\infty}^{+\infty}\left[\cos (k y) \cos \left(m \omega_{0} t\right)+\sin (k y) \sin \left(m \omega_{0} t\right)\right] .
$$

The second term inside the sum can be removed since:

$$
\sum_{m=-\infty}^{+\infty} \sin \left(m \omega_{0} t\right)=0
$$

Using the Fourier series of the Dirac delta function with period $T=2 \pi / \omega_{0}$, we can verify that:

$$
\sum_{m=-\infty}^{+\infty} \cos \left(m \omega_{0} t\right)=2 \pi \sum_{m=-\infty}^{+\infty} \delta\left(\omega_{0} t-2 \pi m\right)
$$

Substituting (A.8) in (A.6), we have:

$$
\langle H(x, y, t)\rangle_{\varphi}=\left\langle H_{0}(x)\right\rangle_{\varphi}+\frac{2 \pi A}{B_{0}} J_{0}(\hat{\rho}) \cos (k y) \sum_{m=-\infty}^{+\infty} \delta\left(\omega_{0} t-2 \pi m\right) .
$$

According to the the gyro-averaged Hamiltonian system (2.6), we have:

$$
\dot{x}=-\partial_{y}\langle H(x, y, t)\rangle_{\varphi}=\frac{2 \pi k A}{\omega_{0} B_{0}} J_{0}(\hat{\rho}) \sin (k y) \sum_{m=-\infty}^{+\infty} \delta(t-m T)
$$

and:

$$
\dot{y}=\partial_{x}\left\langle H_{0}(x)\right\rangle_{\varphi}=\Omega(x)
$$

which defines the frequency of the system, as already mentioned in chapter 2 .

Let $x_{n}$ and $y_{n}$ define the coordinates of the particle at a time instant infinitesimally smaller than $\tau_{n}=n T$, i. e.:

$$
\begin{aligned}
& x_{n}=x\left(\tau_{n}-\varepsilon\right) \\
& y_{n}=y\left(\tau_{n}-\varepsilon\right),
\end{aligned}
$$


where $n=0,1,2, \ldots$ and $\varepsilon \rightarrow 0^{+}$.

According to (A.10), the radial coordinate $x$ has a jump at every time instant $\tau_{n}$ due to the impulsive terms. However, at any other time instant, $x$ is a constant because $\dot{x}=0$. Thus,

$$
x(t)= \begin{cases}x_{n}, & \tau_{n-1}<t<\tau_{n} \\ x_{n+1}, & \tau_{n}<t<\tau_{n+1} .\end{cases}
$$

Assuming a well-behaved function for $H_{0}(x)$ such that $\Omega(x)$ is continuous, Eq. (A.11) implies that $y(t)$ is also a continuous function, what means that $y_{n}=y\left(\tau_{n}-\varepsilon\right)=y\left(\tau_{n}+\right.$ $\varepsilon)=y\left(\tau_{n}\right)$.

Considering the continuity condition for $y(t)$ and integrating (A.10) from $\tau_{n}-\varepsilon$ to $\tau_{n+1}-\varepsilon$ result in:

$$
x_{n+1}=x_{n}+\frac{2 \pi k A}{\omega_{0} B_{0}} J_{0}(\hat{\rho}) S_{n}
$$

where $S_{n}$ is defined by:

$$
S_{n}=\sum_{m=-\infty}^{+\infty} \int_{\tau_{n}-\varepsilon}^{\tau_{n+1}-\varepsilon} \sin (k y) \delta(t-m T) d t=\sin \left(k y_{n}\right) .
$$

The integral in A.16 is zero for values of Tm outside the interval $\left(\tau_{n}-\varepsilon, \tau_{n+1}-\varepsilon\right)$. Tm belongs to the interval $\left(\tau_{n}-\varepsilon, \tau_{n+1}-\varepsilon\right)$ only if $m=n$. In this case, the integral in A.16 is equal to $\sin \left(k y_{n}\right)$. Thus, (A.15) can be rewritten as:

$$
x_{n+1}=x_{n}+\frac{2 \pi k A}{\omega_{0} B_{0}} J_{0}(\hat{\rho}) \sin \left(k y_{n}\right),
$$

Applying (A.14) and integrating (A.11) from $\tau_{n}-\varepsilon$ to $\tau_{n+1}-\varepsilon$ yield:

$$
\begin{aligned}
y_{n+1} & =y_{n}+\int_{\tau_{n}-\varepsilon}^{\tau_{n+1}-\varepsilon} \Omega[x(t)] d t \\
& =y_{n}+\int_{\tau_{n}-\varepsilon}^{\tau_{n}} \Omega\left(x_{n}\right) d t+\int_{\tau_{n}}^{\tau_{n+1}-\varepsilon} \Omega\left(x_{n+1}\right) d t \\
& =y_{n}+\varepsilon \Omega\left(x_{n}\right)+\frac{2 \pi}{\omega_{0}} \Omega\left(x_{n+1}\right) \\
& =y_{n}+\frac{2 \pi}{\omega_{0}} \Omega\left(x_{n+1}\right)
\end{aligned}
$$

Equations (A.17) and (A.18) correspond to the gyro-averaged drift wave map, introduced in chapter 2 . 


\section{Appendix B}

\section{Indicator Points}

As already discussed in chapter 2, if the orbit computed using a indicator point as initial condition is quasiperiodic orbit, this orbit fills a one-dimensional curve which is commonly called shearless curve. In this chapter, we show the necessary conditions and computations to determine the indicator points' formulas The procedure adopted here is based on [40].

\section{B.1 GSNM Indicator Points}

In this section, we obtain the formulas of the indicator points for the GSNM. The GSNM are given by Eqs. (2.29) and (2.30).

\section{B.1.1 Involutions and Symmetry}

The map (2.29)-(2.30) can be described by the transformation $T$, where:

$$
T\left[\begin{array}{l}
I \\
\tilde{\theta}
\end{array}\right]=\left[\begin{array}{ccc}
I-b J_{0}(\hat{\rho}) \sin \left(2 \pi \widetilde{\theta}_{n}\right) & \\
\widetilde{\theta}_{n}+f\left(I-b J_{0}(\hat{\rho}) \sin \left(2 \pi \widetilde{\theta}_{n}\right)\right) & \bmod & 1
\end{array}\right]
$$

where:

$$
f(I)=a\left[\left(1-\frac{\bar{\rho}^{2}}{2}\right)-I^{2}\right]
$$


The map $T$ is time-reversal symmetric with respect to two involutions $I_{0}$ and $I_{1}$, i.e., $T=I_{1} I_{0}$, and $I_{0}^{2}=I_{1}^{2}=\mathbb{I}$, where:

$$
\begin{aligned}
& I_{0}\left[\begin{array}{l}
I \\
\tilde{\theta}
\end{array}\right]=\left[\begin{array}{ccc}
I-b J_{0}(\hat{\rho}) \sin (2 \pi \tilde{\theta}) \\
-\tilde{\theta} & \bmod & 1
\end{array}\right] \\
& I_{1}\left[\begin{array}{l}
I \\
\tilde{\theta}
\end{array}\right]=\left[\begin{array}{ccc}
I & \\
-\tilde{\theta}+f(I) & \bmod & 1
\end{array}\right]
\end{aligned}
$$

The map $T$ is also symmetric under the $S$, i.e. $S T=T S$, defined by:

$$
S\left[\begin{array}{l}
I \\
\tilde{\theta}
\end{array}\right]=\left[\begin{array}{ccc}
-I & \\
\tilde{\theta}-\frac{1}{2} & \bmod & 1
\end{array}\right]
$$

Let's now verify the relations $T=I_{1} I_{0}, I_{0}^{2}=I_{1}^{2}=\mathbb{I}$, and $S T=T S$.

Decomposition of T: $T=I_{1} I_{0}$

$$
\begin{aligned}
I_{1} I_{0}\left[\begin{array}{l}
I \\
\tilde{\theta}
\end{array}\right] & =I_{1}\left[\begin{array}{c}
I-b J_{0}(\hat{\rho}) \sin (2 \pi \tilde{\theta}) \\
-\widetilde{\theta}
\end{array}\right] \\
& =\left[\begin{array}{c}
I-b J_{0}(\hat{\rho}) \sin (2 \pi \tilde{\theta}) \\
\tilde{\theta}+f\left(I-b J_{0}(\hat{\rho}) \sin (2 \pi \widetilde{\theta})\right)
\end{array}\right] \\
& =T
\end{aligned}
$$

The transformations $I_{0}$ and $I_{1}$ are involutions: $I_{0}^{2}=I_{1}^{2}=\mathbb{I}$

$$
\begin{aligned}
I_{0}^{2}\left[\begin{array}{l}
I \\
\tilde{\theta}
\end{array}\right] & =I_{0}\left[\begin{array}{c}
I-b J_{0}(\hat{\rho}) \sin (2 \pi \tilde{\theta}) \\
-\widetilde{\theta}
\end{array}\right] \\
& =\left[\begin{array}{c}
\left(I-b J_{0}(\hat{\rho}) \sin (2 \pi \widetilde{\theta})\right)-b J_{0}(\hat{\rho}) \sin (-2 \pi \widetilde{\theta}) \\
\widetilde{\theta}
\end{array}\right] \\
& =\left[\begin{array}{l}
I \\
\widetilde{\theta}
\end{array}\right]
\end{aligned}
$$




$$
\begin{aligned}
I_{1}^{2}\left[\begin{array}{l}
I \\
\tilde{\theta}
\end{array}\right] & =I_{1}\left[\begin{array}{c}
I \\
-\widetilde{\theta}+f(I)
\end{array}\right] \\
& =\left[\begin{array}{c}
I \\
-(-\tilde{\theta}+f(I))+f(I)
\end{array}\right] \\
& =\left[\begin{array}{c}
I \\
\tilde{\theta}
\end{array}\right]
\end{aligned}
$$

The transformation $S$ is a symmetry of $T: S T=T S$

$$
\begin{aligned}
S T\left[\begin{array}{l}
I \\
\tilde{\theta}
\end{array}\right]=S\left[\begin{array}{c}
I-b J_{0}(\hat{\rho}) \sin \left(2 \pi \widetilde{\theta}_{n}\right) \\
\tilde{\theta}_{n}+f\left(I-b J_{0}(\hat{\rho}) \sin \left(2 \pi \widetilde{\theta}_{n}\right)\right)
\end{array}\right] \\
=\left[\begin{array}{c}
-I+b J_{0}(\hat{\rho}) \sin \left(2 \pi \widetilde{\theta}_{n}\right) \\
\left(\widetilde{\theta}_{n}+f\left(I-b J_{0}(\hat{\rho}) \sin \left(2 \pi \widetilde{\theta}_{n}\right)\right)\right)-\frac{1}{2}
\end{array}\right] \\
T S\left[\begin{array}{l}
I \\
\widetilde{\theta}
\end{array}\right]=T\left[\begin{array}{c}
-I \\
\tilde{\theta}-\frac{1}{2}
\end{array}\right] \\
=\left[\begin{array}{c}
-I-b J_{0}(\hat{\rho}) \sin \left(2 \pi \widetilde{\theta}_{n}-\pi\right) \\
\widetilde{\theta}_{n}-\frac{1}{2}+f\left(-I-b J_{0}(\hat{\rho}) \sin \left(2 \pi \widetilde{\theta}_{n}-\pi\right)\right)
\end{array}\right] \\
=\left[\begin{array}{l}
-I+b J_{0}(\hat{\rho}) \sin \left(2 \pi \widetilde{\theta}_{n}\right) \\
\widetilde{\theta}_{n}-\frac{1}{2}+f\left(-I+b J_{0}(\hat{\rho}) \sin \left(2 \pi \widetilde{\theta}_{n}\right)\right)
\end{array}\right] \\
=S T\left[\begin{array}{l}
I \\
\tilde{\theta}
\end{array}\right]
\end{aligned}
$$

\section{B.1.2 Fixed Points of $S I_{0}$ and $S I_{1}$}

Let's now determine the fixed points of $S I_{0}$ and $S I_{1}$, which we call $z_{0}$ and $z_{1}$, respectively. The points $z_{0}$ and $z_{1}$ are indicator points of the map (2.29)-(2.30). 
If $z_{0}=\left[\begin{array}{c}I_{0} \\ \widetilde{\theta}_{0}\end{array}\right]$, we have:

$$
\begin{aligned}
S I_{0}\left[\begin{array}{l}
I_{0} \\
\widetilde{\theta}_{0}
\end{array}\right] & =\left[\begin{array}{l}
I_{0} \\
\widetilde{\theta}_{0}
\end{array}\right] \\
S\left[\begin{array}{c}
I_{0}-b J_{0}(\hat{\rho}) \sin \left(2 \pi \widetilde{\theta}_{0}\right) \\
-\widetilde{\theta}_{0}
\end{array}\right] & =\left[\begin{array}{c}
I_{0} \\
\tilde{\theta}_{0}
\end{array}\right] \\
{\left[\begin{array}{c}
-I_{0}+b J_{0}(\hat{\rho}) \sin \left(2 \pi \widetilde{\theta}_{0}\right) \\
-\widetilde{\theta}_{0}-\frac{1}{2}
\end{array}\right] } & =\left[\begin{array}{c}
I_{0} \\
\tilde{\theta}_{0}
\end{array}\right]
\end{aligned}
$$

Using (B.6) and considering that $\widetilde{\theta}_{0}$ is periodic inside the interval $[0,1)$, we have:

$$
\begin{aligned}
& \widetilde{\theta}_{0}^{m}=\left(-\widetilde{\theta}_{0}^{m}-\frac{1}{2}\right)+m, \quad m \in \mathbb{Z} \\
& \widetilde{\theta}_{0}^{m}=\frac{m}{2}-\frac{1}{4} \quad \bmod 1
\end{aligned}
$$

And:

$$
\begin{aligned}
& I_{0}^{m}=-I_{0}^{m}+b J_{0}(\hat{\rho}) \sin \left[2 \pi\left(\frac{m}{2}-\frac{1}{4}\right)\right] \\
& I_{0}^{m}=(-1)^{m+1} \frac{b J_{0}(\hat{\rho})}{2}
\end{aligned}
$$

In practice, we just need to consider the solutions for $m=1\left(z_{0}^{1}\right)$ and $m=2\left(z_{0}^{2}\right)$ because all other ones are equivalent.

$$
\begin{gathered}
\widetilde{\theta}_{0}^{1}=\frac{1}{4} \quad \widetilde{\theta}_{0}^{2}=\frac{3}{4} \\
I_{0}^{1}=+\frac{b J_{0}(\hat{\rho})}{2} \\
\widetilde{I}_{0}^{2}=-\frac{b J_{0}(\hat{\rho})}{2}
\end{gathered}
$$

Therefore,

$$
z_{0}^{1}=\left[\begin{array}{c}
+\frac{b J_{0}(\hat{\rho})}{2} \\
\frac{1}{4}
\end{array}\right] \quad z_{0}^{1}=\left[\begin{array}{c}
-\frac{b J_{0}(\hat{\rho})}{2} \\
\frac{3}{4}
\end{array}\right]
$$




$$
\begin{aligned}
& \text { Now let's determine the fixed point } z_{1}=\left[\begin{array}{l}
I_{1} \\
\widetilde{\theta}_{1}
\end{array}\right]: \\
& \qquad \begin{aligned}
{\left[\begin{array}{l}
I_{1} \\
\widetilde{\theta}_{1}
\end{array}\right] } & =S I_{1}\left[\begin{array}{l}
I_{1} \\
\widetilde{\theta}_{1}
\end{array}\right] \\
& =S\left[\begin{array}{c}
I_{1} \\
-\widetilde{\theta}_{1}+f\left(I_{1}\right)
\end{array}\right] \\
& =\left[\begin{array}{c}
-I_{1} \\
-\widetilde{\theta}_{1}+f\left(I_{1}\right)-\frac{1}{2}
\end{array}\right]
\end{aligned}
\end{aligned}
$$

Using (B.10), we see that $I_{1}=0$. Considering that $\widetilde{\theta}_{1}$ is periodic inside the interval $[0,1)$, we also have:

$$
\begin{aligned}
& \widetilde{\theta}_{1}^{m}=\left(-\widetilde{\theta}_{1}^{m}+f(0)-\frac{1}{2}\right)+m, \quad m \in \mathbb{Z} \\
& \widetilde{\theta}_{1}^{m}=\frac{f(0)}{2}+\frac{m}{2}-\frac{1}{4} \bmod 1
\end{aligned}
$$

Again, just the solutions $m=1\left(z_{1}^{1}\right)$ and $m=2\left(z_{2}^{2}\right)$ are sufficient:

$$
z_{1}^{1}=\left[\begin{array}{ccc}
0 & & \\
\frac{a}{2}\left(1-\frac{\bar{\rho}^{2}}{2}\right)+\frac{1}{4} & \bmod & 1
\end{array}\right] \quad z_{1}^{2}=\left[\begin{array}{ccc}
0 & & \\
\frac{a}{2}\left(1-\frac{\bar{\rho}^{2}}{2}\right)+\frac{3}{4} & \bmod & 1
\end{array}\right]
$$

\section{B.2 GQNM Indicator Points}

Using the same procedure presented in section B.1, we can see that the indicator points for the GQNM are given by the following formulas:

$$
\begin{array}{cc}
z_{0}^{1}=\left[\begin{array}{c}
+\frac{b J_{0}(\hat{\rho})}{2} \\
\frac{1}{4}
\end{array}\right] \quad z_{0}^{2}=\left[\begin{array}{cc}
-\frac{b J_{0}(\hat{\rho})}{2} \\
\frac{3}{4}
\end{array}\right] \\
z_{1}^{1}=\left[\begin{array}{ccc}
0 & \\
\frac{f(0)}{2}+\frac{1}{4} & \bmod & 1
\end{array}\right] & z_{1}^{2}=\left[\begin{array}{ccc}
0 & \\
\frac{f(0)}{2}+\frac{3}{4} & \bmod & 1
\end{array}\right]
\end{array}
$$


where $f(I)$ is given by:

$$
f(I)=a\left(1-I^{2}\right)\left\{1-\bar{\rho}^{2}\left[\frac{3}{2}\left(1-I^{2}\right)-1\right]\right\}
$$

Thus, $f(0)=a\left(1-\frac{\bar{\rho}^{2}}{2}\right)$ and:

$$
z_{1}^{1}=\left[\begin{array}{ccc}
0 & & \\
\frac{a}{2}\left(1-\frac{\bar{\rho}^{2}}{2}\right)+\frac{1}{4} & \bmod & 1
\end{array}\right] \quad z_{1}^{2}=\left[\begin{array}{ccc}
0 & & \\
\frac{a}{2}\left(1-\frac{\bar{\rho}^{2}}{2}\right)+\frac{3}{4} & \bmod & 1
\end{array}\right]
$$

Note that the indicator point formulas are the same ones of the GNSM. 


\section{Appendix $\mathrm{C}$}

\section{Statistics of Gamma: Further}

\section{Results}

In this chapter we provide an alternative way to obtain the probability density function, the moments and the cumulative distribution function associated to $\gamma$, that we call here also perturbative ratio. An addition discussion about properties of the perturbative ratio's pdf is presented.

\section{C.1 Alternative form of the Gamma's PDF}

Let normalized Larmor radius be described by the random variable $X$, which can assume any value $\widehat{\rho}$ in the domain $D=[0,+\infty) . X-P D F$ is defined by:

$$
f_{X}(\widehat{\rho})=\frac{\widehat{\rho}}{\widehat{\rho}_{t h}^{2}} \exp \left[-\frac{1}{2}\left(\frac{\widehat{\rho}}{\widehat{\rho}_{t h}}\right)^{2}\right] .
$$

Let $Y$ be defined as the random variable associated to the perturbative ratio $\gamma=$ $K / K_{0}$. Thus:

$$
Y=u(X)
$$

where $u: D \longmapsto \mathbb{R}$ and $u(\widehat{\rho})=J_{0}(\widehat{\rho})$. Function $u$ corresponds to the zero-order Bessel function defined on a non-negative real domain.

Let $\left\{\left(\widehat{\rho}_{k}, \gamma_{k}=u\left(\widehat{\rho}_{k}\right)\right)\right\}_{k=0}^{\infty}$ be defined as the set of points of maximum and minimum of $u$, and $D_{k}$ as the interval $\left[\widehat{\rho}_{k}, \widehat{\rho}_{k+1}\right)$. The set of intervals $\left\{D_{k}\right\}_{k=0}^{\infty}$ is a partition of the 


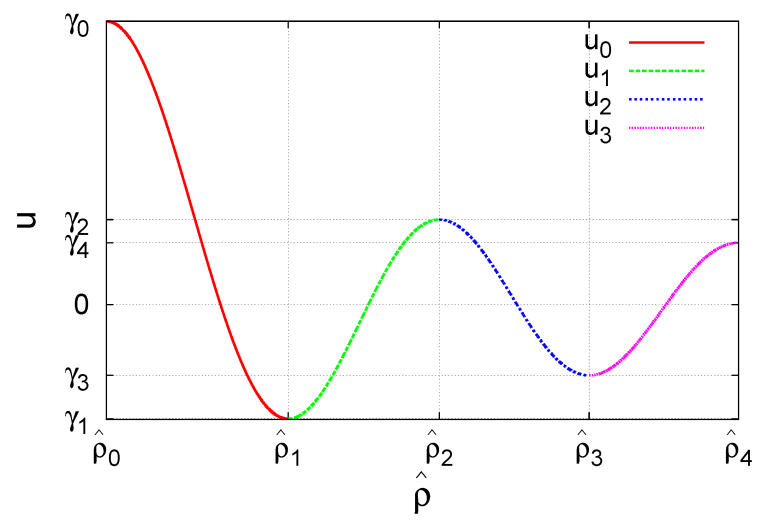

Figure C.1: Partition of the function $\gamma$ in the the set of monotonic functions $\left\{u_{k}\right\}_{k=0}^{\infty}$. Each function $u_{k}$ is defined between points of maximum and minimum of $\gamma$. Approximate coordinates of the maxima and minima shown in the figure are $\left(\widehat{\rho}_{0}, \gamma_{0}\right) \simeq(0,1),\left(\widehat{\rho}_{1}, \gamma_{1}\right) \simeq(3.83171,-0.402759),\left(\widehat{\rho}_{2}, \gamma_{2}\right) \simeq(7.01559,0.300116)$, and $\left(\widehat{\rho}_{3}, \gamma_{3}\right) \simeq(10.1735,0.218359)$.

domain $D$ in such a way that all functions $u_{k}$, defined by $u_{k}: D_{k} \longmapsto\left[\gamma_{k}, \gamma_{k+1}\right)$ and $u_{k}(\widehat{\rho})=u(\widehat{\rho})$, are monotonic, as shown in Fig. C.1. Functions $u_{k}$ are increasing for even values of $k$ and decreasing for odd values of $k$.

A way to determine the probability density function of the perturbative ratio ( $Y$-PDF) is through the distribution function of $Y$ :

$$
F_{Y}(\gamma)=P(Y \leq \gamma)
$$

which means the probability that $Y \leq \gamma$. The $Y$-PDF, that we shall decribe by the function $f_{Y}(\gamma)$, corresponds to the first derivative of $F_{Y}(\gamma)$ :

$$
f_{Y}(\gamma)=F_{Y}^{\prime}(\gamma)
$$

According to (C.3) and (C.4):

$$
F_{Y}(\gamma)=\int_{-\infty}^{\gamma} f_{Y}\left(\gamma^{\prime}\right) d \gamma^{\prime}
$$

Since $\left\{D_{k}\right\}_{k=0}^{\infty}$ is a partition of the sample space of $X$, the probability (C.3) can be written as:

$$
F_{Y}(\gamma)=\sum_{k=0}^{\infty} P\left(Y \leq \gamma \cap X \in D_{k}\right)
$$


where $P\left(Y \leq \gamma \cap X \in D_{k}\right)$ is the probability of the simultaneous occurrence of the events $Y \leq \gamma$ and $X \in D_{k}$. Let $P\left(Y \leq \gamma \mid X \in D_{k}\right)$ be the conditional probability of $Y \leq \gamma$ given that $X \in D_{k}$. The distribution (C.6) is then:

$$
F_{Y}(\gamma)=\sum_{k=0}^{\infty} F_{Y}^{(k)}(\gamma)
$$

with $F_{Y}^{(k)}(\gamma)$ defined as:

$$
F_{Y}^{(k)}(\gamma)=P\left(Y \leq \gamma \mid X \in D_{k}\right) P\left(X \in D_{k}\right)
$$

where $P\left(X \in D_{k}\right)$ is the probability of $X \in D_{k}$, given by:

$$
P\left(X \in D_{k}\right)=\int_{\widehat{\rho}_{k}}^{\widehat{\rho}_{k+1}} f_{X}(\widehat{\rho}) d \widehat{\rho}
$$

which, using the distribution function of $X, F_{X}(\widehat{\rho})$, can also be written as:

$$
P\left(X \in D_{k}\right)=F_{X}\left(\widehat{\rho}_{k+1}\right)-F_{X}\left(\widehat{\rho}_{k}\right)
$$

To determine the PDF of $Y$, we need to compute, according to (C.4), the derivative of (C.7):

$$
f_{Y}(\gamma)=\sum_{k=0}^{\infty} f_{Y}^{(k)}(\gamma)
$$

where $f_{Y}^{(k)}(\gamma)$ is defined by:

$$
f_{Y}^{(k)}(\gamma)=\frac{d}{d \gamma}\left[F_{Y}^{(k)}(\gamma)\right]
$$

Let $a_{k}=\min \left\{\gamma_{k}, \gamma_{k+1}\right\}$ and $b_{k}=\max \left\{\gamma_{k}, \gamma_{k+1}\right\}$, which correspond to the minimum and maximum of $u(\widehat{\rho})$ in the interval $\left[\widehat{\rho}_{k}, \widehat{\rho}_{k+1}\right]$. In case of even $k$ (decreasing $u_{k}$ ), the minimum is $a_{k}=\gamma_{k+1}$ and the maximum $b_{k}=\gamma_{k}$. In case of odd $k$ (increasing $u_{k}$ ), the minimum is $a_{k}=\gamma_{k}$ and the maximum $b_{k}=\gamma_{k+1}$. The terms $f_{Y}^{(k)}(\gamma)$ in (C.11) are computed in two different ways depending on $u_{k}(\widehat{\rho})$ is an increasing or a decreasing function. 


\section{$f_{Y}^{(k)}(\gamma)$ for decreasing $u_{k}(\widehat{\rho})$}

Depending on the value $\gamma$ of the random variable $Y$, the probability $P\left(Y \leq \gamma \mid X \in D_{k}\right)$ assume three different values:

$$
P\left(Y \leq \gamma \mid X \in D_{k}\right)=\left\{\begin{array}{cc}
1 & \gamma \in\left[b_{k},+\infty\right) \\
P\left[u_{k}^{-1}(\gamma) \leq X<\widehat{\rho}_{k+1} \mid X \in D_{k}\right] & \gamma \in\left(a_{k}, b_{k}\right) \\
0 & \gamma \in\left(-\infty, a_{k}\right]
\end{array}\right.
$$

where $u_{k}^{-1}(\gamma)$ is the inverse function of $u_{k}(\widehat{\rho})$. Substituting (C.13) in (C.8), we have:

$$
F_{Y}^{(k)}(\gamma)=\left\{\begin{array}{cc}
P\left(X \in D_{k}\right) & \gamma \in\left[b_{k},+\infty\right) \\
P\left[u_{k}^{-1}(\gamma) \leq X<\widehat{\rho}_{k+1} \mid X \in D_{k}\right] P\left(X \in D_{k}\right) & \gamma \in\left(a_{k}, b_{k}\right) \\
0 & \gamma \in\left(-\infty, a_{k}\right]
\end{array}\right.
$$

Since:

$$
\begin{aligned}
P\left[u_{k}^{-1}(\gamma) \leq X<\widehat{\rho}_{k+1} \mid X \in D_{k}\right] P\left(X \in D_{k}\right) & =P\left[u_{k}^{-1}(\gamma) \leq X<\widehat{\rho}_{k+1} \cap X \in D_{k}\right] \\
& =P\left[u_{k}^{-1}(\gamma) \leq X<\widehat{\rho}_{k+1}\right] \\
& =F_{X}\left(\widehat{\rho}_{k+1}\right)-F_{X}\left[u_{k}^{-1}(\gamma)\right]
\end{aligned}
$$

and knowing that $P\left(X \in D_{k}\right)$ and $F_{X}\left(\widehat{\rho}_{k+1}\right)$ don't depend on $\gamma$, the derivative of (C.14) is:

$$
f_{Y}^{(k)}(\gamma)=\left\{\begin{array}{cc}
-\frac{f_{X}\left[u_{k}^{-1}(\gamma)\right]}{u_{k}^{\prime}\left[u_{k}^{-1}(\gamma)\right]} & \gamma \in\left(a_{k}, b_{k}\right) \\
0 & \gamma \notin\left(a_{k}, b_{k}\right)
\end{array}\right.
$$

where $u_{k}^{\prime}(\gamma)$ is the derivative of $u_{k}(\gamma)$.

\section{$f_{Y}^{(k)}(\gamma)$ for increasing $u_{k}(\widehat{\rho})$}

For decreasing $u_{k}(\widehat{\rho})$, the probability $P\left(Y \leq \gamma \mid X \in D_{k}\right)$ assume the following values:

$$
P\left(Y \leq \gamma \mid X \in D_{k}\right)=\left\{\begin{array}{cc}
1 & \gamma \in\left[b_{k},+\infty\right) \\
P\left[\widehat{\rho}_{k} \leq X \leq u_{k}^{-1}(\gamma) \mid X \in D_{k}\right] & \gamma \in\left(a_{k}, b_{k}\right) \\
0 & \gamma \in\left(-\infty, a_{k}\right]
\end{array}\right.
$$


Substituting (C.16) in (C.8), we have:

$$
F_{Y}^{(k)}(\gamma)=\left\{\begin{array}{cc}
P\left(X \in D_{k}\right) & \gamma \in\left[b_{k},+\infty\right) \\
P\left[\widehat{\rho}_{k} \leq X \leq u_{k}^{-1}(\gamma) \mid X \in D_{k}\right] P\left(X \in D_{k}\right) & \gamma \in\left(a_{k}, b_{k}\right) \\
0 & \gamma \in\left(-\infty, a_{k}\right]
\end{array}\right.
$$

The value of $F_{Y}^{(k)}(\gamma)$ for $\gamma \in\left(a_{k}, b_{k}\right)$ is:

$$
\begin{aligned}
P\left[\widehat{\rho}_{k} \leq X \leq u_{k}^{-1}(\gamma) \mid X \in D_{k}\right] P\left(X \in D_{k}\right) & =P\left[\widehat{\rho}_{k} \leq X \leq u_{k}^{-1}(\gamma) \cap X \in D_{k}\right] \\
& =P\left[\widehat{\rho}_{k} \leq X \leq u_{k}^{-1}(\gamma)\right] \\
& =F_{X}\left[u_{k}^{-1}(\gamma)\right]-F_{X}\left(\widehat{\rho}_{k}\right)
\end{aligned}
$$

Since $F_{X}\left(\widehat{\rho}_{k}\right)$ in (C.18) and $P\left(X \in D_{k}\right)$ in (C.17) are constants, the derivative of (C.17) can be written as:

$$
f_{Y}^{(k)}(\gamma)=\left\{\begin{array}{cc}
+\frac{f_{X}\left[u_{k}^{-1}(\gamma)\right]}{u_{k}^{\prime}\left[u_{k}^{-1}(\gamma)\right]} & \gamma \in\left(a_{k}, b_{k}\right) \\
0 & \gamma \notin\left(a_{k}, b_{k}\right)
\end{array}\right.
$$

\section{PDF}

Inside the interval $\left(a_{k}, b_{k}\right), u_{k}^{\prime}(\gamma)<0$ for decreasing $u_{k}(\gamma)$ and $u_{k}(\gamma)>0$ for increasing $u_{k}(\gamma)$, which means that equations (C.15) and (C.19) can be written in only one formula:

$$
f_{Y}^{(k)}(\gamma)=\left\{\begin{array}{cc}
+\frac{f_{X}\left[u_{k}^{-1}(\gamma)\right]}{\left|u_{k}^{\prime}\left[u_{k}^{-1}(\gamma)\right]\right|} & \gamma \in\left(a_{k}, b_{k}\right) \\
0 & \gamma \notin\left(a_{k}, b_{k}\right)
\end{array}\right.
$$

The probability density function of the perturbative ratio, which corresponds to the PDF of the random variable $Y$, is thus defined by (C.11):

$$
f_{Y}(\gamma)=\sum_{k=0}^{\infty} f_{Y}^{(k)}(\gamma)
$$

where $f_{Y}^{(k)}(\gamma)$ is given by (C.20), which can be rewritten as:

$$
f_{Y}^{(k)}(\gamma)=\left\{\begin{array}{cc}
+\frac{u_{k}^{-1}(\gamma)}{\widehat{\rho}_{t h}^{2}} \frac{\exp \left\{-\frac{1}{2}\left[\frac{u_{k}^{-1}(\gamma)}{\hat{\rho}_{t h}}\right]^{2}\right\}}{\left|J_{1}\left[u_{k}^{-1}(\gamma)\right]\right|} & \gamma \in\left(a_{k}, b_{k}\right) \\
0 & \gamma \notin\left(a_{k}, b_{k}\right)
\end{array}\right.
$$




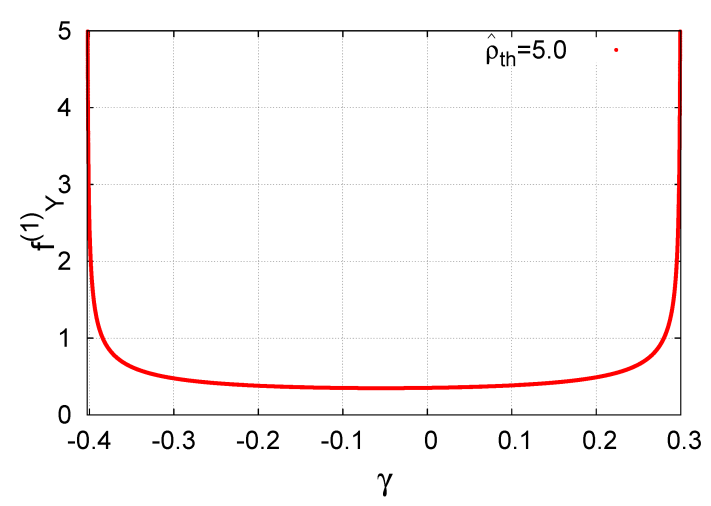

Figure C.2: $f_{Y}^{(1)}(\gamma)$ for $\widehat{\rho}_{t h}=5.0$

Figure C.2 shows a plot of $f_{Y}^{(1)}(\gamma)$ for $\widehat{\rho}_{t h}=5.0$, one of the terms $f_{Y}^{(k)}(\gamma)$, given by (C.22). Outside the interval $\left(a_{1}, b_{1}\right)$, where $\left(a_{1} \simeq-0.4\right.$ and $\left.b_{1} \simeq+0.3\right), f_{Y}^{(1)}(\gamma)$ is equal to zero. Otherwise, $f_{Y}^{(1)}(\gamma)$ is different from zero and goes to infinity when $\gamma \rightarrow a_{1}^{+}$and $\gamma \rightarrow b_{1}^{-}$. In Fig. C.2, the "peaks" near -0.4 and +0.3 result from singularities associated to $f_{Y}^{(1)}(\gamma)$.

Figure C.3 shows plots of $f_{Y}(\gamma)$ for different values of the thermal Larmor radius, $\hat{\rho}_{t h}$. The plots exhibit the combined effect of all terms $f_{Y}^{(k)}(\gamma)$. Some terms $f_{Y}^{(k)}(\gamma)$ have a dominant effect, what means that the corresponding peaks are clearly visible. The peaks are located near the maxima and minima of $J_{0}$, which correspond to the singularites of $f_{Y}(\gamma)$.

\section{Singularities}

For increasing $u_{k}$ :

$$
\lim _{\gamma \rightarrow a_{k}^{+}} f_{X}\left[u_{k}^{-1}(\gamma)\right]=f_{X}\left(\widehat{\rho}_{k}\right), \quad \lim _{\gamma \rightarrow b_{k}^{-}} f_{X}\left[u_{k}^{-1}(\gamma)\right]=f_{X}\left(\widehat{\rho}_{k+1}\right)
$$

For decreasing $u_{k}$ :

$$
\lim _{\gamma \rightarrow a_{k}^{+}} f_{X}\left[u_{k}^{-1}(\gamma)\right]=f_{x}\left(\widehat{\rho}_{k+1}\right), \quad \lim _{\gamma \rightarrow b_{k}^{-}} f_{X}\left[u_{k}^{-1}(\gamma)\right]=f_{X}\left(\widehat{\rho}_{k}\right)
$$

Since $a_{k}$ and $b_{k}$ are, respectively, the minimum and maximum of $u_{k}$, we have:

$$
\lim _{\gamma \rightarrow a_{k}^{+}}\left|u_{k}^{\prime}\left[u_{k}^{-1}(\gamma)\right]\right|=0, \quad \lim _{\gamma \rightarrow b_{k}^{-}}\left|u_{k}^{\prime}\left[u_{k}^{-1}(\gamma)\right]\right|=0
$$




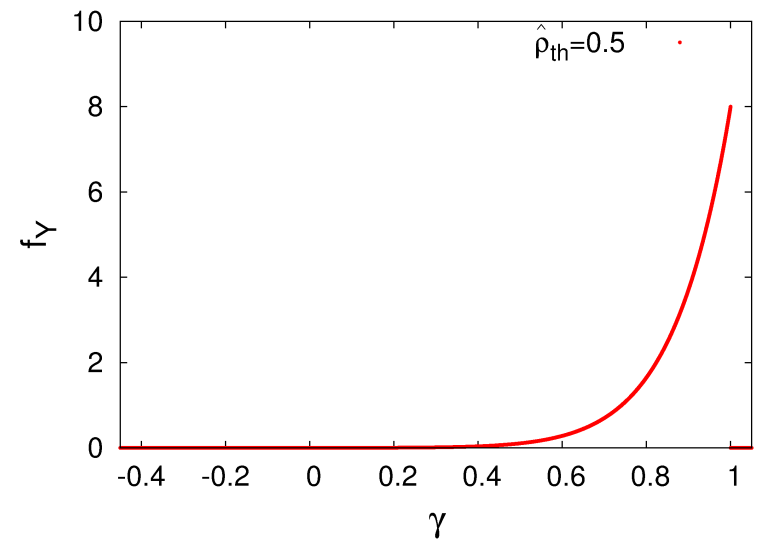

(a) $\hat{\rho}_{t h}=0.5$

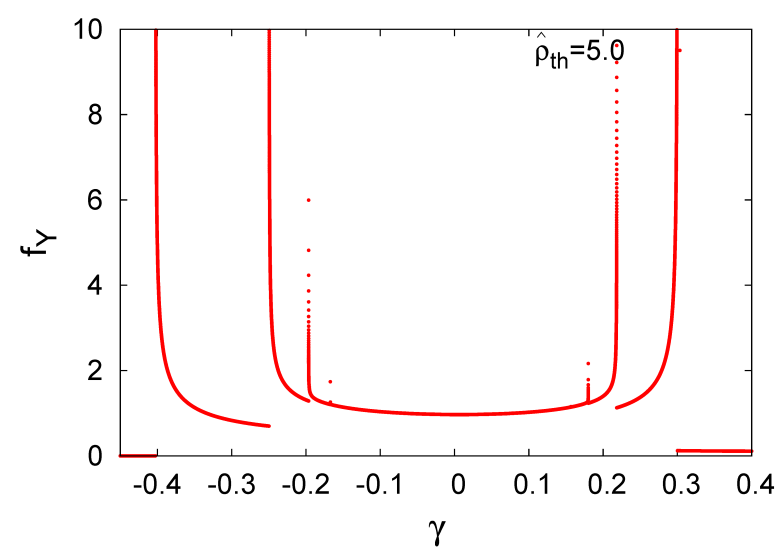

(c) $\hat{\rho}_{t h}=5.0$

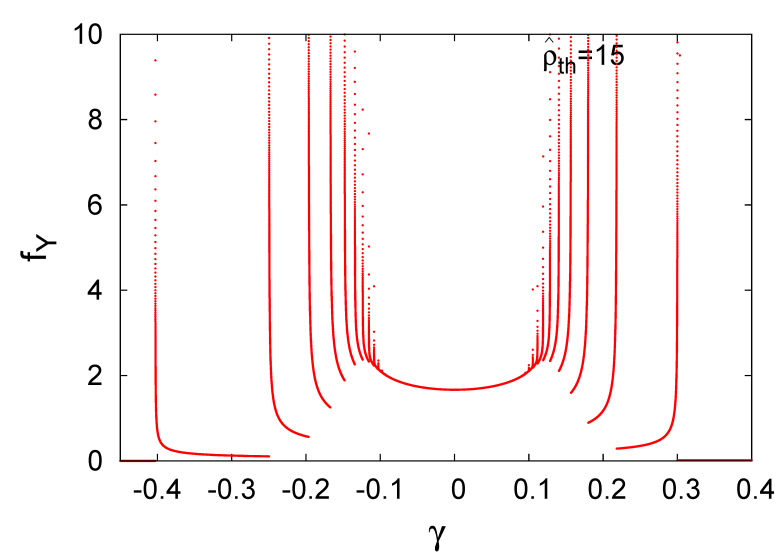

(e) $\hat{\rho}_{t h}=15$

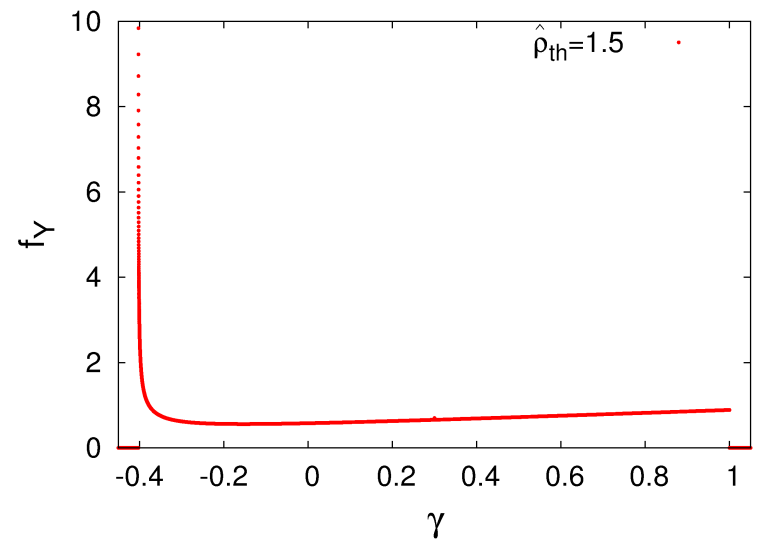

(b) $\hat{\rho}_{t h}=1.5$

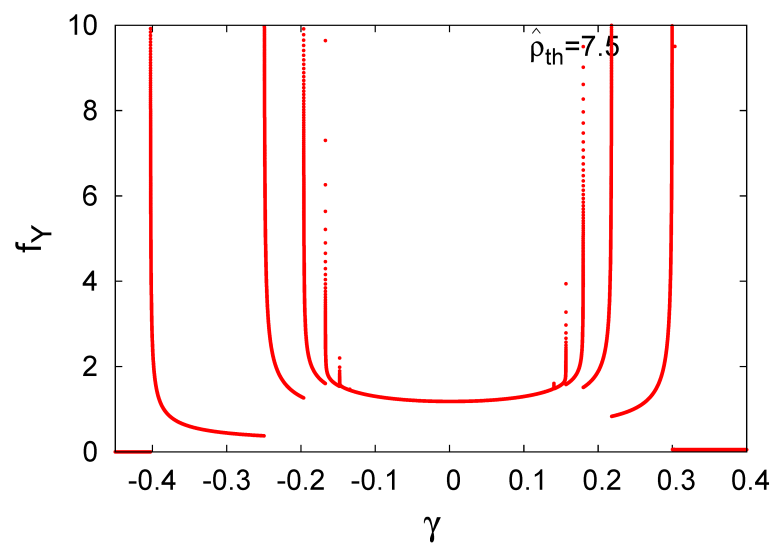

(d) $\hat{\rho}_{t h}=7.5$

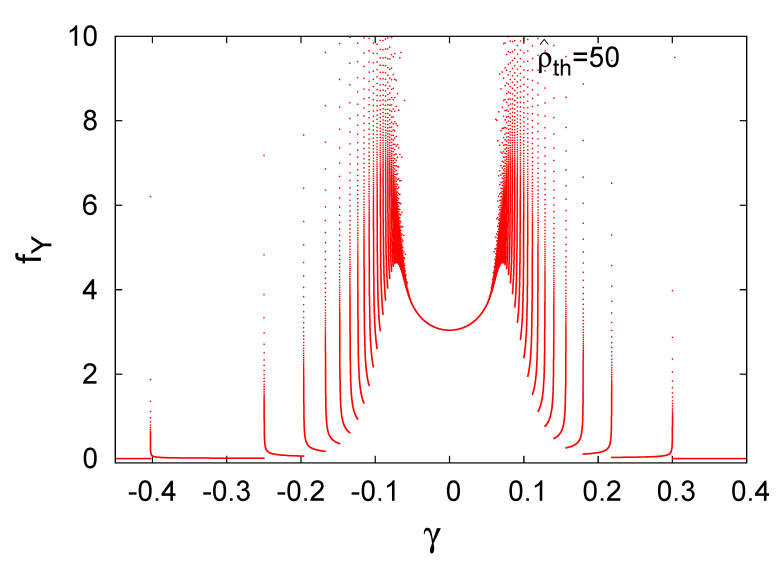

(f) $\hat{\rho}_{t h}=50$

Figure C.3: Plots of $f_{Y}(\gamma)$ for different values of $\widehat{\rho}_{t h}$. The singularities associated to the terms $f_{Y}^{(k)}(\gamma)$ terms explain the high values of $f_{Y}(\gamma)$ near the maxima and minima of $J_{0}$. 
From Eqs. (C.23), (C.24), and (C.25), we see that $f_{Y}^{(k)}(\gamma)$ diverges positively when $\gamma \rightarrow a_{k}^{+}$for any $k$ and also when $\gamma \rightarrow b_{k}^{-}$for $k>0$. However, according to (C.20), $f_{Y}^{(k)}(\gamma)$ drops to zero outside the interval $\left(a_{k}, b_{k}\right)$ for any $k$. That is, $f_{Y}^{(k)}(\gamma)=0$ for $\gamma \in\left(-\infty, a_{k}\right] \bigcup\left[b_{k},+\infty\right)$.

There's no divergence in $f_{Y}^{(0)}(\gamma)$ when $\gamma \rightarrow b_{0}^{-}=1^{-}$. From (C.22) and (C.24), we have:

$$
\begin{aligned}
\lim _{\gamma \rightarrow 1^{-}} f_{Y}^{(0)}(\gamma) & =\lim _{\widehat{\rho} \rightarrow 0^{+}} \frac{\widehat{\rho}}{\widehat{\rho}_{t h}^{2}} \frac{\exp \left[-\frac{1}{2}\left(\frac{\widehat{\rho}}{\hat{\rho}_{t h}}\right)^{2}\right]}{\left|J_{1}(\widehat{\rho})\right|} \\
& =\frac{2}{\widehat{\rho}_{t h}^{2}}
\end{aligned}
$$

since $J_{1}(\widehat{\rho}) \sim \frac{\widehat{\rho}}{2}$ for small arguments. Therefore, $f_{Y}^{(0)}(\gamma)$ does not diverge if $\gamma \rightarrow b_{0}^{-}$(the only case in all set of terms $\left.f_{Y}^{(k)}(\gamma)\right)$ but diverges if $\gamma \rightarrow a_{0}^{+}$.

\section{C.2 Moments}

The $n$-th moment of $Y$ is given by:

$$
\left\langle Y^{n}\right\rangle=\int_{-\infty}^{+\infty} \gamma^{n} f_{Y}(\gamma) d \gamma
$$

Substituting (C.21) in (C.27), we have:

$$
\left\langle Y^{n}\right\rangle=\sum_{k=0}^{\infty} \int_{\gamma_{k}}^{\gamma_{k+1}} \gamma^{n} \frac{f_{X}\left[u_{k}^{-1}(\gamma)\right]}{\left|u_{k}^{\prime}\left[u_{k}^{-1}(\gamma)\right]\right|} d \gamma
$$

for increasing $u_{k}$, and:

$$
\left\langle Y^{n}\right\rangle=\sum_{k=0}^{\infty} \int_{\gamma_{k+1}}^{\gamma_{k}} \gamma^{n} \frac{f_{X}\left[u_{k}^{-1}(\gamma)\right]}{\left|u_{k}^{\prime}\left[u_{k}^{-1}(\gamma)\right]\right|} d \gamma
$$

for decreasing $u_{k}$.

Since $\left|u_{k}^{\prime}\right|=+u_{k}^{\prime}$ for increasing $u_{k}$ and $\left|u_{k}^{\prime}\right|=-u_{k}^{\prime}$ for decreasing $u_{k}$, Eqs. (C.28) and (C.29) can be written in just one formula:

$$
\left\langle Y^{n}\right\rangle=\sum_{k=0}^{\infty} \int_{\gamma_{k}}^{\gamma_{k+1}} \gamma^{n} \frac{f_{X}\left[u_{k}^{-1}(\gamma)\right]}{u_{k}^{\prime}\left[u_{k}^{-1}(\gamma)\right]} d \gamma
$$


Let $\widehat{\rho}=u_{k}^{-1}(\gamma)$, which means that $\gamma=u_{k}(\widehat{\rho})=u(\widehat{\rho})$. Changing the integration variable in (C.30), we obtain:

$$
\left\langle Y^{n}\right\rangle=\int_{0}^{+\infty}[u(\widehat{\rho})]^{n} f_{X}(\widehat{\rho}) d \widehat{\rho}
$$

which, according to (C.1), is the same as:

$$
\left\langle Y^{n}\right\rangle=\frac{1}{\widehat{\rho}_{t h}^{2}} \int_{0}^{+\infty}\left[J_{0}(\widehat{\rho})\right]^{n} \exp \left[-\frac{1}{2}\left(\frac{\widehat{\rho}}{\widehat{\rho}_{t h}}\right)^{2}\right] \widehat{\rho} d \widehat{\rho} .
$$

Formula (C.32) corresponds to Eq. (3.30), obtained in chapter 3 by a different procedure.

\section{C.3 Cumulative Distribution Function}

According to (C.5), the distribution function of $Y$ is:

$$
F_{Y}(\gamma)=\sum_{k=0}^{\infty} \int_{-\infty}^{\gamma} f_{Y}^{(k)}\left(\gamma^{\prime}\right) d \gamma^{\prime}
$$

where $f_{Y}^{(k)}$ is defined by (C.20).

Let $I_{k}(\gamma)$ be defined by the integral in (C.33):

$$
I_{k}(\gamma)=\int_{-\infty}^{\gamma} f_{Y}^{(k)}\left(\gamma^{\prime}\right) d \gamma^{\prime}
$$

$I_{k}(\gamma)$ can then assume the following expressions:

$$
I_{k}(\gamma)=\left\{\begin{array}{cc}
\int_{a_{k}}^{b_{k}} \frac{f_{X}\left[u_{k}^{-1}\left(\gamma^{\prime}\right)\right]}{\left|u_{k}^{\prime}\left[u_{k}^{-1}\left(\gamma^{\prime}\right)\right]\right|} d \gamma^{\prime} & \gamma \geq b_{k} \\
\int_{a_{k}}^{\gamma} \frac{f_{X}\left[u_{k}^{-1}\left(\gamma^{\prime}\right)\right]}{\left|u_{k}^{\prime}\left[u_{k}^{-1}\left(\gamma^{\prime}\right)\right]\right|} d \gamma^{\prime} & \gamma \in\left(a_{k}, b_{k}\right) \\
0 & \gamma \leq a_{k}
\end{array}\right.
$$

In case of decreasing $u_{k}$ functions, let $\gamma^{\prime}=u_{k}(\widehat{\rho})$ and $I_{k}(\gamma)=I_{k}^{-}(\gamma)$. According to (C.35), $I_{k}^{-}(\gamma)$ is given by:

$$
I_{k}^{-}(\gamma)=\left\{\begin{array}{cc}
\int_{\widehat{\rho}_{k+1}}^{\widehat{\rho}_{k}} f_{X}(\widehat{\rho}) \frac{u_{k}^{\prime}(\hat{\rho})}{\left|u_{k}^{\prime}(\hat{\rho})\right|} d \widehat{\rho}, & \gamma \geq b_{k} \\
\int_{\widehat{\rho}_{k+1}}^{u_{k}^{-1}(\gamma)} f_{X}(\widehat{\rho}) \frac{u_{k}^{\prime}(\hat{\rho})}{\left|u_{k}^{\prime}(\hat{\rho})\right|} d \widehat{\rho}, & \gamma \in\left(a_{k}, b_{k}\right) \\
0 & \gamma \leq a_{k}
\end{array}\right.
$$


Since $u_{k}$ is decreasing, we have:

$$
\frac{u_{k}^{\prime}(\hat{\rho})}{\left|u_{k}^{\prime}(\hat{\rho})\right|}=-1
$$

$I_{k}^{-}(\gamma)$ can then be rewritten as:

$$
I_{k}^{-}(\gamma)=\left\{\begin{array}{cc}
\int_{\widehat{\rho}_{k}}^{\widehat{\rho}_{k+1}} f_{X}(\widehat{\rho}) d \widehat{\rho}, & \gamma \geq b_{k} \\
\int_{u_{k}^{-1}(\gamma)}^{\widehat{\rho}_{k+1}} f_{X}(\widehat{\rho}) d \widehat{\rho}, & \gamma \in\left(a_{k}, b_{k}\right) \\
0, & \gamma \leq a_{k}
\end{array}\right.
$$

If $\gamma \geq b_{k}, \gamma-u_{k}(\hat{\rho})>0$ and $\Theta\left(\gamma-u_{k}(\hat{\rho})\right)=1$ for any $\hat{\rho} \in\left(\rho_{k}, \hat{\rho}_{k+1}\right)$, where $\Theta$ corresponds to the Heaviside step function. Thus,

$$
\int_{\widehat{\rho}_{k}}^{\widehat{\rho}_{k+1}} f_{X}(\widehat{\rho}) d \widehat{\rho}=\int_{\widehat{\rho}_{k}}^{\widehat{\rho}_{k+1}} \Theta\left[\gamma-u_{k}(\hat{\rho})\right] f_{X}(\widehat{\rho}) d \widehat{\rho}, \quad \gamma \geq b_{k}
$$

If $\gamma \in\left(a_{k}, b_{k}\right), \gamma-u_{k}(\hat{\rho})>0$ and $\Theta\left[\gamma-u_{k}(\hat{\rho})\right]=1$ for any $\hat{\rho} \in\left(u_{k}^{-1}(\gamma), \hat{\rho}_{k+1}\right)$. For $\hat{\rho} \in\left(\hat{\rho}_{k}, u_{k}^{-1}(\gamma)\right), \gamma-u_{k}(\hat{\rho})<0$ and $\Theta\left[\gamma-u_{k}(\hat{\rho})\right]=0$. Thus, the second case in (C.38) can then also be written as:

$$
\begin{aligned}
\int_{u_{k}^{-1}(\gamma)}^{\widehat{\rho}_{k+1}} f_{X}(\widehat{\rho}) d \widehat{\rho} & =\int_{\widehat{\rho}_{k}}^{u_{k}^{-1}(\gamma)} \Theta\left[\gamma-u_{k}(\hat{\rho})\right] f_{X}(\widehat{\rho}) d \widehat{\rho}+\int_{u_{k}^{-1}(\gamma)}^{\widehat{\rho}_{k+1}} \Theta\left[\gamma-u_{k}(\hat{\rho})\right] f_{X}(\widehat{\rho}) d \widehat{\rho}( \\
& =\quad \int_{\widehat{\rho}_{k}}^{\widehat{\rho}_{k+1}} \Theta\left[\gamma-u_{k}(\hat{\rho})\right] f_{X}(\widehat{\rho}) d \widehat{\rho}, \quad \gamma \in\left(a_{k}, b_{k}\right)
\end{aligned}
$$

If $\gamma \leq a_{k}, \gamma-u_{k}(\hat{\rho})<0$ and $\Theta\left[\gamma-u_{k}(\hat{\rho})\right]=0$ for any $\hat{\rho} \in\left(\hat{\rho}_{k}, \hat{\rho}_{k+1}\right)$. The third case in (C.38)has then also the same form as in the first and second cases (Eqs.(C.39) and (C.41)).

Therefore,

$$
I_{k}^{-}(\gamma)=\int_{\widehat{\rho}_{k}}^{\widehat{\rho}_{k+1}} \Theta\left[\gamma-u_{k}(\hat{\rho})\right] f_{X}(\widehat{\rho}) d \widehat{\rho}
$$

for every real $\gamma$.

Let $I_{k}(\gamma)=I_{k}^{+}(\gamma)$ when $u_{k}$ is increasing. Changing again the integration variable from $\gamma^{\prime}$ to $\hat{\rho}=u_{k}^{-1}\left(\gamma^{\prime}\right), I_{k}^{+}(\gamma)$, according to (C.35), is given by:

$$
I_{k}^{+}(\gamma)=\left\{\begin{array}{cc}
\int_{\rho_{k}}^{\rho_{k+1}} f_{X}(\hat{\rho}) \frac{u_{k}^{\prime}(\hat{\rho})}{\left|u_{k}^{\prime}(\hat{\rho})\right|} d \widehat{\rho} & \gamma \geq b_{k} \\
\int_{\rho_{k}}^{u_{k}^{-1}(\gamma)} f_{X}(\hat{\rho}) \frac{u_{k}^{\prime}(\hat{\rho})}{\left|u_{k}^{\prime}(\hat{\rho})\right|} d \widehat{\rho} & \gamma \in\left(a_{k}, b_{k}\right) \\
0 & \gamma \leq a_{k}
\end{array}\right.
$$


where:

$$
\frac{u_{k}^{\prime}(\hat{\rho})}{\left|u_{k}^{\prime}(\hat{\rho})\right|}=+1
$$

for increasing $u_{k}$ functions.

The same procedure used to obtain Eq. (C.42) can be applied to obtain $I_{k}^{+}(\gamma)$. Again, if $\gamma \geq b_{k}, \Theta\left[\gamma-u_{k}(\hat{\rho})\right]=1$ for all increasing $u_{k}$ functions and every $\hat{\rho} \in\left(\rho_{k}, \hat{\rho}_{k+1}\right)$. And also, if $\gamma \leq a_{k}, \Theta\left[\gamma-u_{k}(\hat{\rho})\right]=0$ for all increasing $u_{k}$ functions and every $\hat{\rho} \in\left(\rho_{k}, \hat{\rho}_{k+1}\right)$. Therefore, the first and third cases in (C.43) can be written as:

$$
I_{k}^{+}(\gamma)=\int_{\widehat{\rho}_{k}}^{\widehat{\rho}_{k+1}} \Theta\left[\gamma-u_{k}(\hat{\rho})\right] f_{X}(\widehat{\rho}) d \widehat{\rho}, \quad \gamma \in\left(-\infty, a_{k}\right] \bigcup\left[b_{k},+\infty\right)
$$

If $\gamma \in\left(a_{k}, b_{k}\right), \Theta\left[\gamma-u_{k}(\hat{\rho})\right]=1$ for $\hat{\rho} \in\left(\hat{\rho}_{k}, u_{k}^{-1}(\gamma)\right)$ and $\Theta\left[\gamma-u_{k}(\hat{\rho})\right]=0$ for $\hat{\rho} \in\left(u_{k}^{-1}(\gamma), \hat{\rho}_{k+1}\right)$. Thus, the second case in (C.43) can be written as:

$$
\begin{aligned}
\int_{\rho_{k}}^{u_{k}^{-1}(\gamma)} f_{X}(\hat{\rho}) d \widehat{\rho} & =\int_{\widehat{\rho}_{k}}^{u_{k}^{-1}(\gamma)} \Theta\left[\gamma-u_{k}(\hat{\rho})\right] f_{X}(\widehat{\rho}) d \widehat{\rho}+\int_{u_{k}^{-1}(\gamma)}^{\widehat{\rho}_{k+1}} \Theta\left[\gamma-u_{k}(\hat{\rho})\right] f_{X}(\widehat{\rho}) d \widehat{\rho}(\mathrm{C} .46) \\
& =\quad \int_{\widehat{\rho}_{k}}^{\widehat{\rho}_{k+1}} \Theta\left[\gamma-u_{k}(\hat{\rho})\right] f_{X}(\widehat{\rho}) d \widehat{\rho}, \quad \gamma \in\left(a_{k}, b_{k}\right) \quad \text { (C.47) }
\end{aligned}
$$

From (C.45) and (C.47), we see that:

$$
I_{k}^{+}(\gamma)=\int_{\widehat{\rho}_{k}}^{\widehat{\rho}_{k+1}} \Theta\left[\gamma-u_{k}(\hat{\rho})\right] f_{X}(\widehat{\rho}) d \widehat{\rho}
$$

which means that the same formula applies to both $I_{k}^{+}(\gamma)$ and $I_{k}^{-}(\gamma)$.

According to (C.33) and (C.34), the distribution function associated to the random variable $Y$ is given by:

$$
F_{Y}(\gamma)=\sum_{k=0}^{\infty} I_{k}(\gamma)
$$

where, since $I_{k}^{+}(\gamma)$ (Eq. (C.48)) and $I_{k}^{-}(\gamma)$ (Eq. (C.42)) are described by the same formula, $I_{k}(\gamma)$ is:

$$
I_{k}(\gamma)=\int_{\widehat{\rho}_{k}}^{\widehat{\rho}_{k+1}} \Theta\left[\gamma-u_{k}(\hat{\rho})\right] f_{X}(\widehat{\rho}) d \widehat{\rho}
$$

Thus,

$$
\begin{aligned}
F_{Y}(\gamma) & =\sum_{k=0}^{\infty} \int_{\widehat{\rho}_{k}}^{\widehat{\rho}_{k+1}} \Theta\left[\gamma-u_{k}(\hat{\rho})\right] f_{X}(\widehat{\rho}) d \widehat{\rho} \\
& =\int_{\widehat{\rho}_{0}}^{\widehat{\rho}_{1}} \Theta\left[\gamma-u_{k}(\hat{\rho})\right] f_{X}(\widehat{\rho}) d \widehat{\rho}+\int_{\widehat{\rho}_{1}}^{\widehat{\rho}_{2}} \Theta\left[\gamma-u_{k}(\hat{\rho})\right] f_{X}(\widehat{\rho}) d \widehat{\rho}+\int_{\widehat{\rho}_{2}}^{\widehat{\rho}_{3}} \Theta\left[\gamma-u_{k}(\hat{\rho})\right] f_{X}(\widehat{\rho}) d \widehat{\rho}+\ldots
\end{aligned}
$$


which means that:

$$
F_{Y}(\gamma)=\int_{0}^{\infty} \Theta[\gamma-u(\hat{\rho})] f_{X}(\hat{\rho}) d \hat{\rho}
$$

Formula (C.53) corresponds to Eq. (3.39), obtained in chapter 3 by a different procedure. 


\section{Bibliography}

[1] R. G. Kleva, and J. F. Drake, Phys. Fluids 27, 1686 (1984).

[2] M. Pettini, A. Vulpiani, et al. Phys. Rev. A 38, 344 (1988)

[3] F. A. Marcus, I. L. Caldas, Z. O Guimaraes Filho, P. J. Morrison, W. Horton, Y. K. Kuznetsov and I. L. Nascimento, Phys. Plasmas 15, 112304 (2008).

[4] D. del-Castillo-Negrete, Phys. Plasmas 7, 1702 (2000).

[5] G. Manfredi and R. O. Dendy, Phys. Rev. Lett., 76, 4360 (1996).

[6] J. J. Martinell and D. del-Castillo-Negrete, Phys. Plasmas 20, 022303 (2013).

[7] S. V. Annibaldi, G. Manfredi, and R. O. Dendy, Phys. Plasmas 9, 791 (2002).

[8] G. Manfredi and R. Dendy, Phys. Plasmas 4, 628 (1997).

[9] K. Gustafson, D. del-Castillo-Negrete and W. Dorland, Phys. Plasmas 16, 102309 (2008).

[10] D. del-Castillo-Negrete and J.J. Martinell, Commun. Nonlinear Sci. Numer. Simulat. 17, 2031 (2012).

[11] W. Horton, Plasma Phys. Controlled Fusion 27, 9 (1985).

[12] W. W. Lee, J. Comp. Phys 72, 243 (1987).

[13] W. Horton, H.-B. Park, J.-M. Kwon, D. Strozzi, P. J. Morrison, and D.-I. Choi, Phys. Plasmas 5, 11 (1998). 
[14] R. Egydio de Carvalho and A. M. Ozorio de Almeida. Physics Letters A, 162, 457 (1992).

[15] J. D. da Fonseca, "Barreiras de Transporte em Plasmas e Mapas Simpléticos NãoTwist". Master Dissertation, University of São Paulo (2013).

[16] G. A. Oda and I. L. Caldas, Chaos, Solitons and Fractals, 515 (1995).

[17] R. Balescu, Phys. Rev. E 58, 3 (1998).

[18] D. del-Castillo-Negrete and P. J. Morrison, Bull. Am. Phys. Soc., Series II, 37, 1543 (1992)

[19] D. del-Castillo-Negrete and P. J. Morrison, Phys. Fluids A 5, 948 (1993)

[20] F. J. Beron-Vera, M. J. Olascoaga, M. G. Brown MG, et al. Chaos 20, 017514 (2010).

[21] M. V. Budyansky, M. Yu. Uleysky, and S. V. Prants, Phys. Rev. E, 79, 056215 (2009).

[22] J. D. Meiss, Rev. Mod. Phys. 64, 3 (1992).

[23] I. I. Rypina, M. G. Brown, et al. Phys. Rev. Lett. 98, 104102 (2007).

[24] J. S. E. Portela, I. L. Caldas et al. International Journal of Bifurcation and Chaos in Applied Sciences and Engineering, 17, 1589, (2007).

[25] J. D. Szezech, I. L. Caldas, et al. Chaos: An Interdisciplinary Journal of Nonlinear Science, 19, 043108 (2009).

[26] I. L. Caldas, R.L. Viana et al. Commun. Nonlinear Sci. Numer. Simulat. 17, 2021 (2011)

[27] A. Delshams and R. de-la-Llave, SIAM J. Math. Anal., 31, 1235 (2000)

[28] E. G. Altmann, G. Cristadoro, and D. Pazó, Phys. Rev. E, 73, 056201 (2006).

[29] N. Slater, Proc. Cambridge Philos. Soc. 63, 1115 (1967).

[30] Y. Zou, D. Pazó, et al. Phys. Rev. E, 76, 016210 (2007). 
[31] C.V. Abud and I.L. Caldas, Physica D: Nonlinear Phenomena, 308, 34 (2015).

[32] B. V. Chirikov, Phys. Rep., 52, 263, (1979).

[33] J. B. Taylor, Culham Lab. Prog. Report CLM-PR-12, (1969).

[34] J. D. da Fonseca, D. del-Castillo-Negrete, and I.L. Caldas, Phys. of Plasmas, 21, 92310 (2014).

[35] D. R Nicholson, "Introduction to Plasma Theory". John Wiley and Sons, Inc. New York, 1983.

[36] L. Nasi and M.-C. Firpo, Plasma Physics and Controlled Fusion, 51, 045006 (2009).

[37] J.M Greene, J. Math. Phys. 20, 1183 (1979)

[38] S. Shinohara and Y. Aizawa, Prog. Theor. Phys. 97, 379 (1997).

[39] E. Petrisor, Int. J. Bifurcation Chaos, 11, 497 (2001)

[40] A. Wurm, A. Apte, et al. CHAOS 15, 2023108 (2005).

[41] D. del-Castillo-Negrete, Phys. Fluids 10, 576 (1998).

[42] J. D. da Fonseca, D. del-Castillo-Negrete, I.M. Sokolov and I.L. Caldas. "Statistical Properties of the GSM model", paper to be submitted to the scientific journal Physics of Plasmas.

[43] J. A. Bittencourt. "Fundamentals of Plasmas Physics". Springer-Verlag, 3rd edition, 2004 .

[44] K. Miyamoto. "Plasma Physics and Controlled Nuclear Fusion". Springer-Verlag, 2005

[45] D.T. Gillepsie and E. Seitaridou, "Simple Brownian Diffusion: An Introduction to the Standard Theoretical Models". Oxford University Press, 2013.

[46] R. P. Kanwal, "Generalized Functions: Theory and Applications". Birkhäuser, 2004. 
[47] M. Abramowitz and I. Stegun, "Handbook of Mathematical Functions with Formulas, Graphs, and Mathematical Tables". Dover, 1965.

[48] S. Gradshteyn and I. M. Ryzhik, "Table of Integrals, Series, and Products". Academic Press, 2007.

[49] R. Venegeroles, Phys. Rev. Lett., 101, 054102 (2008). 